Universidade de São Paulo

Instituto de Física

\title{
Influência da Transferência de Momento-Energia na Interação entre Matéria e Energia Escura
}

\author{
Lucas Collis Olivari
}

Orientador: Prof. Dr. Elcio Abdalla

Dissertação de mestrado apresentada ao Instituto de Física para a obtenção do título de Mestre em Ciências

Banca Examinadora:

Prof. Dr. Elcio Abdalla (IF-USP)

Prof. Dr. Luis Raul Weber Abramo (IF-USP)

Prof. Dr. Vilson Tonin Zanchin (UFABC) 
FICHA CATALOGRÁFICA

Preparada pelo Serviço de Biblioteca e Informação do Instituto de Física da Universidade de São Paulo

Olivari, Lucas Collis

Influência da transferência de momento-energia na interação entre matéria e energia escura. São Paulo, 2014.

Dissertação (Mestrado) - Universidade de São Paulo. Instituto de Física. Depto. de Física Matemática

Orientador: Prof. Dr. Elcio Abdalla

Área de Concentração: Física

Unitermos: 1. Cosmologia 2. ; Matéria escura;

3. Energia escura.

USP/IF/SBI-029/2014 


\section{Agradecimentos}

Agradeço, primeiramente, ao Prof. Dr. Elcio Abdalla. Além de orientar-me durante o mestrado, também possibilitou que eu vivenciasse um ambiente de pesquisa de alto nível.

Agradeço aos colegas do grupo de Cosmologia, não só pela ajuda no trabalho diário, mas também pela companhia diária.

Agradeço, também, aos meus grandes amigos do saudoso tempo de Graduação na Unicamp. Sem eles, talvez, não teria terminado minha Graduação em Física, muito menos começado o Mestrado na mesma.

Agradeço, principalmente, à minha família. Meu pai, Luis, minha mãe, Tânia, minha irmã, Giovanna, e minha avó, Mirctes. A velha citação que diz que a família é o alicerce do homem finalmente fez sentido a mim durante esses anos em São Paulo.

Por fim, agradeço à FAPESP pelo apoio financeiro. 



\section{Resumo}

Neste trabalho, estudamos modelos cosmológicos em que a energia escura foi tratada como um campo de matéria que interage com a matéria escura. Três modelos distintos foram considerados. O primeiro trata tanto a matéria escura fria quanto a energia escura como fluidos perfeitos. O termo de interação entre eles é dado por uma expressão com origem fenomenológica que postulamos existir na equação de balanço entre esses dois fluidos. Dadas as equações no universo plano de Friedmann-Robertson-Walker (FRW), pudemos escrever uma versão covariante para as equações de balanço. Com isso, as equações de balanço em um universo de FRW perturbado linearmente foram obtidas. Isso, por sua vez, permitiu que a estabilidade das equações diferenciais obtidas fosse estudada.

O segundo modelo tem origem em modelos de $f(R)$. Esses modelos propõem uma generalização da Relatividade Geral ao considerar a ação da gravidade como um funcional do escalar de Ricci, $R$. Através de uma transformação conforme, foi possível reinterpretar os modelos de $f(R)$ como modelos em que um campo escalar canônico, que representa a energia escura, interage com os campos da matéria. Através do princípio da ação, obtivemos as equações de movimento e o tensor de energia-momento para nosso sistema. Com o campo escalar sendo interpretado como um fluido perfeito, pudemos, por fim, obter equações de balanço entre fluidos perfeitos tanto no nível de fundo quanto no universo perturbado linearmente.

O terceiro modelo começa com a lagrangiana, em um espaço-tempo de FRW, de um campo escalar canônico, que representa a energia escura, e um campo fermiônico de spin- $\frac{1}{2}$, que representa a matéria escura. Um termo de interação de Yukawa entre esses campos foi postulado existir na lagrangiana. Novamente através do princípio da ação, obtivemos as equações de movimento e o tensor de energia-momento para esses campos. Essas equações de movimento puderam, por fim, ser reescritas como equações de balanço entre fluidos perfeitos tanto no nível de fundo quanto no universo perturbado linearmente. 



\begin{abstract}
In this work we studied cosmological models in which the dark energy was treated as a field of matter that interacts with dark matter. Three different models were considered. The first one treats both the cold dark matter and the dark energy as perfect fluids. The interaction term between them is given by a expression with phenomenological origin that we postulated to exist in the balance equations between these two fluids. Given the equations in the flat Friedmann-Robertson-Walker (FRW) universe, we wrote a covariant version of the balance equations. Thus, the balance equations in a linearly perturbed FRW universe were obtained. This, in turn, allowed the stability of the obtained differential equations to be studied.

The second model comes from $f(R)$ models. These models propose a generalization of General Relativity by considering the action for gravity as a functional of the Ricci scalar, $R$. Through a conformal transformation, it was possible to reinterpret the $f(R)$ models as models in which a canonical scalar field, which represents the dark energy, interacts with matter fields. Through the principle of least action, we obtained the equations of motion and the energy-momentum tensor for our system. With the scalar field being interpreted as a perfect fluid, we obtained equations of balance for perfect fluids at both the background level and in the linearly perturbed universe.

The third model starts with the Lagrangian, in a FRW space-time, of a canonical scalar field, which represents the dark energy, and of a fermionic field of spin- $\frac{1}{2}$, which represents the dark matter. A Yukawa interaction term between these fields was postulated to exist in the Lagrangian. Again, through the principle of least action, we obtained the equations of motion and the energy-momentum tensor for these fields. These equations of motion could then be rewritten as balance equations for perfect fluids at both the background level and in the linearly perturbed universe.
\end{abstract}





\section{Sumário}

1 Introdução 5

2 Modelo Padrão da Cosmologia 9

2.1 Equações de Friedmann . . . . . . . . . . . . . . . . . . . . . . 10

2.2 Lei de Hubble . . . . . . . . . . . . . . . . . . . . . . . . . . . . . . . 14

2.3 Componentes que Compõem a Matéria do Universo . . . . . . . . . . 15

2.3.1 Componentes Relativísticos . . . . . . . . . . . 16

2.3.2 Componentes Não-Relativísticos . . . . . . . . . . . . . . 18

2.4 Distâncias Cósmicas . . . . . . . . . . . . . . . . . . . . . 20

2.4.1 Distância Comóvel . . . . . . . . . . . . . . . . 21

2.4 .2 Distância de Luminosidade . . . . . . . . . . . . . . . . . 22

2.4.3 Distância do Diâmetro Angular . . . . . . . . . . . . . . . . . 23

2.5 A Equação de Estado da Energia Escura . . . . . . . . . . . . . . . . 23

3 Teoria de Perturbação $\quad 27$

3.1 Perturbações Lineares na Relatividade Geral . . . . . . . . . . . . . . 27

3.2 Gauge Newtoniano . . . . . . . . . . . . . . . . . . . . . . . 28

3.3 Modelo Cosmológico com um Único Fluido . . . . . . . . . . . . . . . 32

3.3.1 Escalas Maiores do que o Horizonte . . . . . . . . . . . . . . . 37

3.3.2 Escalas Menores do que o Horizonte . . . . . . . . . . . . . 38

3.4 Solução Cosmológica com Dois Fluidos . . . . . . . . . . . . . . . . . 40

3.5 Equação de Boltzmann para Bárions, Fótons e Neutrinos . . . . . . . 43

3.6 O Espectro de Potência da Matéria . . . . . . . . . . . . . . . . . . 52

4 Energia Escura $\quad 57$

4.1 Evidências Observacionais da Energia Escura . . . . . . . . . . . . 57

4.1 .1 A Idade do Universo . . . . . . . . . . . . . . . . . . . . 58

4.1 .2 Observações de Supernovas . . . . . . . . . . . . . . . . . . . 59

4.1.3 A Radiação Cósmica de Fundo em Microondas . . . . . . . . . 61

4.2 Constante Cosmológica . . . . . . . . . . . . . . . . . . . . . 69 
4.2.1 Equações de Einstein com uma Constante Cosmológica . . . . 69

4.2.2 O Problema do Ajuste Fino . . . . . . . . . . . . . . . 71

4.2.3 O Problema da Coincidência . . . . . . . . . . . . 72

4.3 Quintessência . . . . . . . . . . . . . . . 73

4.3 .1 Sistema Dinâmico . . . . . . . . . . . . . . . 76

5 Interação no Setor Escuro na Linguagem de Fluidos Interagentes 81

5.1 Universo Homogêneo e Isotrópico . . . . . . . . . . . . . . . . . . 81

5.1.1 Considerações Termodinâmicas sobre a Interação no Setor Escuro ............................ 85

5.2 Formalismo Perturbativo . . . . . . . . . . . . . . 87

5.2.1 Estabilidade da Perturbação de Curvatura . . . . . . . . . . . 95

6 Interação no Setor Escuro no Formalismo Lagrangiano 101

6.1 Modelo para a Energia Escura Interagindo com a Matéria Escura Oriundo de Modelos de $f(R)$. . . . . . . . . . . . . . . . 101

6.1.1 Modelos de $f(R)$ no Formalismo Métrico . . . . . . . . . . . 102

6.1.2 Equivalência com a Teoria de Brans-Dicke . . . . . . . . . . . 104

6.1.3 Transformação Conforme . . . . . . . . . . . . . . . . . 105

6.1.4 Quintessência Acoplada . . . . . . . . . . . . . . 108

6.2 Modelo de Yukawa para a Energia Escura Interagindo com a Matéria

Escura . . . . . . . . . . . . . . . . . . . . . 112

6.2.1 Formalismo das Tétradas . . . . . . . . . . . . . . . . . 113

6.2.2 Modelo de Yukawa no Universo de FRW . . . . . . . . . . . . 115

6.2.3 Comparação com Dados Observacionais . . . . . . . . . . . . . 120

7 Conclusão 


\section{Capítulo 1}

\section{Introdução}

Cosmologia é o ramo da física que estuda a estrutura e a evolução do universo. Para entendermos as sutilezas e as generalidades do universo, precisamos fazer hipóteses e construir teorias ou modelos para que previsões sejam feitas. Previsões que devem ser postas à prova através de experimentos e de observações. Como o nosso universo é um objeto irreproduzível em laboratório, temos que nos contentar com as observações feitas por nossos telescópios. Contudo, para nosso deleite, essas observações, que avançam em precisão em um ritmo alucinante, são mais do que suficientes para confirmar nossas suspeitas sobre o funcionamento básico do universo, e surpreendentes o bastante para expandir nossos horizontes sobre os detalhes de como o universo surgiu e como ele evoluiu desde o ainda misterioso Big Bang.

Uma dessas expansões de nosso horizonte no que se refere à compreensão do universo é justamente como o universo expande-se atualmente: ele expande-se aceleradamente. No contexto da Relatividade Geral, isto é, não considerando modelos que modificam ou generalizam a Relatividade Geral, a aceleração do universo é dada por um tipo de energia misteriosa, alcunhada de energia escura. Um dos principais e mais sutis candidatos para a energia escura é a constante cosmológica. No entanto, é difícil entender a origem da constante cosmológica em termos das teorias fundamentais da física. O valor observado é muito inferior ao calculado com base nas ferramentas da teoria quântica de campos, fato que é conhecido como o problema da constante cosmológica - a diferença entre o cálculo teórico e a medida observacional é de aproximadamente 120 ordens de grandeza. Além disso, usando a constante cosmológica para explicar a energia escura, somos inevitavelmente conduzidos para o problema da coincidência, isto é, por que a densidade de energia do vácuo e da matéria são exatamente da mesma ordem hoje? Necessitaríamos, no caso da constante cosmológica, de um ajuste fino nas condições iniciais para explicar essa coincidência e isso é o tipo de explicação que um físico gostaria de evitar se possível. 
Como a energia escura contribui uma fração significativa do conteúdo do universo, é natural, no contexto da teoria quântica de campos, onde a energia escura é um campo de matéria, considerar suas interações com os demais campos. Por exemplo, podemos nos perguntar o que acontece com a evolução do universo se tivermos uma interação entre a energia escura e a matéria escura - outra componente material do universo cuja origem é objeto de intensa pesquisa. A possibilidade de que energia e matéria escura interajam uma com a outra tem sido discutida por vários grupos de pesquisa [1-4]. Demonstrou-se, por exemplo, que uma interação adequada entre elas pode proporcionar um mecanismo para aliviar o problema da coincidência ao fazer com que a razão entre as suas densidades de energia seja constante, pelo menos por partes, durante a história do universo [1,4]. Todas as observações cosmológicas, como a radiação cósmica de fundo, supernovas do tipo Ia, oscilações acústicas de bárions e a distribuição dos aglomerados de galáxias, são compatíveis com uma interação no setor escuro $[4,5]$, o que nos motiva a continuar explorando essa possibilidade e estudando suas consequências.

Assim, o objetivo desta dissertação é estudar como a energia escura comporta-se em um nível elementar e como ela interage com a matéria. Para isso, estudaremos três modelos distintos. Um deles desconsidera totalmente as sutilezas da energia escura. Nesse modelo, a energia escura é tratada como um fluido ideal interagindo com a matéria escura através de um termo de interação com origem fenomenológica. Já os outros dois modelos buscam explicações mais fundamentais para a energia escura. Ambos fazem uso de um campo escalar canônico para descrever a energia escura, porém eles partem de princípios físicos distintos. O primeiro deles olha para a Relatividade Geral e generaliza sua ação de forma a termos um funcional que dependa do escalar de Ricci de forma geral ao invés de ser simplesmente linear nele. Esse tipo de modificação da Relatividade Geral pode ser reinterpretado fisicamente de uma maneira totalmente distinta. Através de uma transformação conforme, a ação do modelo de $f(R)$ pode ser escrita como uma ação de um campo escalar interagindo com a matéria. Da interação entre o campo escalar e a matéria oriunda dessa transformação, podemos obter as equações para a evolução do universo. O outro modelo, que chamaremos de modelo de Yukawa, invoca um campo escalar que tenha origem em algum modelo que vá além do modelo padrão da física de partículas e pergunta o que acontece com a evolução do universo se esse campo escalar interagir com um campo fermiônico, que representa uma matéria escura efetiva, através de uma interação de Yukawa.

Esta dissertação está estruturada em sete capítulos que tratam majoritariamente do problema da energia escura e da possibilidade de interação entre os componentes 
do setor escuro. No segundo capítulo, um resumo do Modelo Padrão da Cosmologia, ou modelo $\Lambda \mathrm{CDM}$, é feito. A ideia desse capítulo é desenvolver os conceitos mais básicos para o desenvolvimento futuro do trabalho e calcular resultados que possam ser úteis mais adiante. No terceiro capítulo, desenvolvemos os principais conceitos e as principais equações da teoria de perturbações lineares para o universo, considerando uma métrica de fundo de Friedmann-Robertson-Walker (FRW) e fluidos perfeitos para a matéria. Nesse capítulo, todos os fundamentos necessários para aplicar a teoria de perturbação linear em modelos com interação no setor escuro são desenvolvidos e discutidos.

O quarto capítulo é sobre a energia escura vista como uma nova componente material do universo. Primeiramente, exploramos as principais observações realizadas até hoje que indicam fortemente a necessidade de uma nova componente da matéria ou uma modificação da Relatividade Geral. Exploramos nesse contexto as supernovas do tipo Ia, a radiação cósmica de fundo em microondas e a idade dos objetos mais antigos do universo. Em seguida, uma solução para energia escura é proposta: a constante cosmológica. Todas as virtudes e falhas da constante cosmológica são então detalhadas. Por fim, o modelo de quintessência, um campo escalar canônico minimamente acoplado à gravidade que faz com que a expansão do universo acelere-se exatamente como nos modelos mais básicos para o universo inflacionário, é discutido, e a sua viabilidade geral explorada.

O quinto capítulo é o primeiro que tratará sobre a interação entre energia e matéria escura. Nesse capítulo, o setor escuro é descrito como sendo composto por fluidos perfeitos. Um ansatz para a interação é proposto e a teoria de perturbação linear desenvolvida, com as modificações em relação ao caso sem interação explicadas. Por fim, a análise da estabilidade das equações diferenciais obtidas é feita, e as consequências cosmológicas discutidas.

O sexto capítulo é sobre os dois modelos que utilizam campos escalares para descrever a energia escura interagindo com a matéria escura. No primeiro modelo, partimos da lagrangiana que modifica a gravidade através de um termo geral que dependa do escalar de Ricci, os chamados modelos de $f(R)$, e através de uma transformação conforme, introduzimos um campo escalar canônico que interage com a matéria do universo. As equações para a evolução são então obtidas e discutidas. O segundo modelo, por sua vez, começa com a lagrangiana de um campo escalar canônico, que representa a energia escura, e um campo fermiônico de spin- $\frac{1}{2}$, que representa a matéria escura, na presença de uma métrica curva (métrica de FRW). Propomos, então, uma interação de tipo Yukawa entre os campos e desenvolvemos as equações de movimento para os campos. Essas equações de movimento podem, por 
sua vez, ser reescritas como equações de balanço entre fluidos, o que permitirá que esse modelo seja futuramente comparado com observações cosmológicas. O último capítulo é a conclusão desta dissertação e a discussão dos trabalhos futuros que dela se originarão. 


\section{Capítulo 2}

\section{Modelo Padrão da Cosmologia}

O universo observável parece, em larga escala, isto é, para distâncias maiores do que aproximadamente 300 milhões de anos-luz, ser o mesmo em todas as direções que observamos. Essa isotropia é diretamente mensurável (aproximadamente uma parte em $10^{5}$ ) quando observamos a radiação cósmica de fundo. Essa radiação cósmica está viajando pelo universo há cerca de 14 bilhões de anos, portanto, ela é um ótimo indicativo de que o universo é realmente o mesmo em qualquer direção quando visto em distâncias suficientemente grandes.

Como é difícil de imaginar que nós estamos em alguma posição especial no universo, podemos concluir que o universo também deve parecer isotrópico para outros observadores no universo. Porém, não para todos eles. O universo não aparece de forma alguma isotrópico para um observador que viaja com, digamos, metade da velocidade da luz. Para esse observador, a radiação cósmica de fundo vinda pela frente em sua direção terá uma intensidade maior do que a radiação cósmica que vem por trás. Assim, ao assumirmos a isotropia do universo, devemos especificar que o universo parece o mesmo em todas as direções somente para uma família de observadores em queda livre, que são aqueles que se movem com a velocidade média das galáxias típicas de sua vizinhança, visto que as galáxias geralmente são parte de um sistema gravitacional estável e fechado. Isso implica que as condições devem ser as mesmas, no mesmo tempo, em qualquer um dos pontos que possa ser levado um ao outro por uma rotação em torno de uma galáxia típica. Mas como todo ponto pode ser levado um ao outro por uma sequência de tais rotações ao redor de galáxias típicas, o universo é também homogêneo - observadores em todas as galáxias típicas em um determinado tempo observam as mesmas condições ao seu redor. A suposição que nosso universo é homogêneo e isotrópico em larga escala permite que um modelo padrão para nosso universo seja proposto e que nossa teoria possa ser comparada com observações. Esse modelo padrão, oriundo de princípios e teorias 
físicas conhecidas, será o tema deste capítulo.

\subsection{Equações de Friedmann}

Para descrever a geometria de nosso universo, precisamos determinar o elemento de linha que descreve o espaço-tempo quadridimensional de um universo homogêneo e isotrópico. Esse elemento de linha é dado pela métrica de Friedmann-RobertsonWalker (FRW) [6],

$$
\mathrm{d} s^{2}=g_{\mu \nu} \mathrm{d} x^{\mu} \mathrm{d} x^{\nu}=-\mathrm{d} t^{2}+a^{2}(t) \mathrm{d} \sigma^{2} .
$$

Nessa equação, $g_{\mu \nu}$ é a métrica, $a(t)$ é o fator de escala no tempo cósmico $t$ e d $\sigma^{2}$ é a métrica puramente espacial independente do tempo e com curvatura $K$ constante,

$$
\mathrm{d} \sigma^{2}=\gamma_{i j} \mathrm{~d} x^{i} \mathrm{~d} x^{j}=\frac{\mathrm{d} r^{2}}{1-K r^{2}}+r^{2}\left(\mathrm{~d} \theta^{2}+\sin ^{2} \theta \mathrm{d} \phi^{2}\right)
$$

onde temos que $K=+1,0,-1$ corresponde a uma geometria fechada, plana e aberta, respectivamente. Na equação (2.1) os índices gregos $\mu$ e $\nu$ vão de 0 a 3, enquanto os índices latinos $i$ e $j$ vão de 1 a 3 . Podemos, além do tempo cósmico $t$, definir o tempo conforme $\eta$,

$$
\eta \equiv \int a^{-1} \mathrm{~d} t
$$

As equações dinâmicas do universo em expansão podem ser derivadas das equações de campo de Einstein. Para isso, precisamos primeiramente calcular os símbolos de Christoffel, que são obtidos através da métrica pela relação [6]

$$
\Gamma_{\nu \lambda}^{\mu}=\frac{1}{2} g^{\mu \alpha}\left(g_{\alpha \nu, \lambda}+g_{\alpha \lambda, \nu}-g_{\nu \lambda, \alpha}\right),
$$

onde $g_{\alpha \nu, \lambda} \equiv \partial g_{\alpha \nu} / \partial x^{\lambda}$. A métrica inversa é definida pela relação $g^{\mu \alpha} g_{\alpha \nu}=\delta_{\nu}^{\mu}$, onde $\delta_{\nu}^{\mu}$ é o delta de Kronecker. Para quantificar a curvatura do espaço-tempo, precisamos do tensor de Ricci, que é definido por

$$
R_{\mu \nu} \equiv \Gamma_{\mu \nu, \alpha}^{\alpha}-\Gamma_{\mu \alpha, \nu}^{\alpha}+\Gamma_{\mu \nu}^{\alpha} \Gamma_{\alpha \beta}^{\beta}-\Gamma_{\mu \beta}^{\alpha} \Gamma_{\alpha \nu}^{\beta} .
$$

A contração do tensor de Ricci define o escalar de Ricci

$$
R=g^{\mu \nu} R_{\mu \nu}
$$

Dessa maneira, o tensor de Einstein pode ser calculado através de 


$$
G_{\mu \nu} \equiv R_{\mu \nu}-\frac{1}{2} g_{\mu \nu} R
$$

Por fim, a dinâmica cosmológica pode ser obtida através das famosas equações de campo de Einstein [6]

$$
G_{\nu}^{\mu}=8 \pi G T_{\nu}^{\mu}
$$

onde $T_{\mu}^{\nu}$ é o tensor de energia-momento de todos os componentes da matéria e a velocidade da luz c é igual à unidade em nossas unidades de medida. Unidades que serão utilizadas em quase toda essa dissertação.

Para a métrica de FRW, (2.1), os componentes dos símbolos de Christoffel diferentes de zero são

$$
\begin{aligned}
& \Gamma_{i j}^{0}=a^{2} H \gamma_{i j}, \quad \Gamma_{0 j}^{i}=\Gamma_{j 0}^{i}=H \delta_{j}^{i}, \\
& \Gamma_{11}^{1}=\frac{K r}{1-K r^{2}}, \quad \Gamma_{22}^{1}=-r\left(1-K r^{2}\right), \quad \Gamma_{33}^{1}=-r\left(1-K r^{2}\right) \sin ^{2} \theta \\
& \Gamma_{33}^{2}=-\sin \theta \cos \theta, \quad \Gamma_{12}^{2}=\Gamma_{21}^{2}=\Gamma_{21}^{2}=\Gamma_{13}^{3}=\Gamma_{31}^{3}=\frac{1}{r}, \quad \Gamma_{23}^{3}=\Gamma_{32}^{2}=\cot \theta
\end{aligned}
$$

onde definimos $H$, o parâmetro de Hubble, que descreve a taxa de expansão do universo, como

$$
H \equiv \dot{a} / a
$$

Um ponto sobre uma variável representará, neste capítulo, uma derivada com respeito ao tempo cósmico $t$.

Das equações (2.5) e (2.6), temos que o tensor e escalar de Ricci são

$$
\begin{gathered}
R_{00}=-3\left(H^{2}+\dot{H}\right), \quad R_{0 i}=R_{i 0}=0, \quad R_{i j}=a^{2}\left(3 H^{2}+\dot{H}+2 K / a^{2}\right) \gamma_{i j}, \\
R=6\left(2 H^{2}+\dot{H}+K / a^{2}\right) .
\end{gathered}
$$

Usando a equação (2.7) juntamente com a relação $G_{\nu}^{\mu}=g^{\mu \alpha} G_{\alpha \nu}$, o tensor de Einstein é dado por

$$
G_{0}^{0}=-3\left(H^{2}+K / a^{2}\right), \quad G_{i}^{0}=G_{0}^{i}=0, \quad G_{j}^{i}=-\left(3 H^{2}+2 \dot{H}+K / a^{2}\right) \delta_{j}^{i} .
$$


No espaço-tempo de FRW, o tensor de energia-momento da matéria de fundo que compõe o universo é restrito pela homogeneidade e isotropia do espaço-tempo a ter a forma de um fluido perfeito [6]

$$
T_{\nu}^{\mu}=(\rho+P) u^{\mu} u_{\nu}+P \delta_{\nu}^{\mu},
$$

onde $u^{\mu}=(-1,0,0,0)$ é a quadrivelocidade do fluido em coordenadas comóveis e $\rho$ e $P$ são funções do tempo. Como as componentes $(00)$ e $(i j)$ de $T_{\nu}^{\mu}$ são $T_{0}^{0}=-\rho$ e $T_{j}^{i}=P \delta_{j}^{i}, \rho$ e $P$ podem ser interpretadas como sendo, respectivamente, a densidade de energia e a pressão do fluido. Das componentes (00) e (ii) das equações de Einstein (2.8), temos que

$$
\begin{gathered}
H^{2}=\frac{8 \pi G}{3} \rho-\frac{K}{a^{2}}, \\
3 H^{2}+2 \dot{H}=-8 \pi G P-\frac{K}{a^{2}} .
\end{gathered}
$$

Eliminando o termo $K / a^{2}$ dessas equações, temos que

$$
\frac{\ddot{a}}{a}=-\frac{4 \pi G}{3}(\rho+3 P) .
$$

Multiplicando a equação (2.15) por $a^{2}$, diferenciando-a no tempo e usando a equação (2.17), temos que

$$
\dot{\rho}+3 H(\rho+P)=0 .
$$

O tensor de Einstein satisfaz as identidades de Bianchi [6]

$$
\nabla_{\mu} G_{\nu}^{\mu} \equiv \frac{\partial G_{\nu}^{\mu}}{\partial x^{\mu}}+\Gamma_{\alpha \mu}^{\mu} G_{\nu}^{\alpha}-\Gamma_{\nu \mu}^{\alpha} G_{\alpha}^{\mu}=0
$$

onde $\nabla_{\mu}$ denota a derivada covariante compatível com a métrica. Das equações de Einstein (2.8), temos que $\nabla_{\mu} T_{\nu}^{\mu}=0$, o que também nos dá a equação (2.18). Por essa razão, a equação (2.18) é conhecida como equação de conservação da densidade de energia do fluido total do universo.

A equação (2.15) pode ser escrita na forma simplificada,

$$
\Omega_{M}+\Omega_{K}=1
$$

onde definimos os parâmetros de densidade como

$$
\Omega_{M} \equiv \frac{8 \pi G \rho}{3 H^{2}}, \quad \Omega_{K} \equiv-\frac{K}{(a H)^{2}} .
$$


Quando nos referirmos aos valores presentes do parâmetro de densidade escreveremos

$$
\Omega_{M}^{(0)}=\frac{8 \pi G \rho^{(0)}}{3 H_{0}^{2}}, \quad \Omega_{K}^{(0)}=-\frac{K}{\left(a_{0} H_{0}\right)^{2}} .
$$

Para evitar confusão futura, devemos identificar nossa notação aqui. Quando nos referirmos à radiação em geral (componentes relativísticas, como fótons e neutrinos), o subscrito será $r$. Quando somente nos referirmos à radiação eletromagnética, usaremos o subscrito $\gamma$. Matéria bariônica será denotada por $b$ e matéria escura por c. Quando, ao invés de energia escura, referirmos somente à constante cosmológica, usaremos $\Lambda$, no caso geral de energia escura, $d$. Para uma componente geral da matéria, usaremos $M$.

Se a expansão do universo estiver desacelerando-se (isto é, $\ddot{a}<0$ ), então o termo de curvatura $\left|\Omega_{K}\right|$ crescerá (porque o termo $a H=\dot{a}$ decresce com o tempo). Isso ocorre a não ser que tenhamos o caso em que o universo é exatamente plano $(K=0)$ desde o começo. Os dados observacionais do WMAP [7], que mediu as anisotropias da radiação cósmica de fundo com grande precisão, limitam a curvatura do presente universo em $-0,0066<\Omega_{K}^{(0)}<0,0011$ com 95\% de confiança. Assim, consideraremos o universo como sendo plano na maior parte desta dissertação.

Agora, consideraremos o caso em que o universo é dominado por um único componente com uma equação de estado dada por

$$
\omega \equiv P / \rho
$$

Se $\omega$ for uma constante, podemos achar a solução da evolução de $\rho$ e de $a$ para um universo plano. Resolvendo as equações (2.15) e (2.18) nesse caso, temos que

$$
\rho \propto a^{-3(1+\omega)}, \quad a \propto\left(t-t_{i}\right)^{2 / 3(1+\omega)}
$$

onde $t_{i}$ é uma constante arbitrária. Veremos que as ferramentas da mecânica estatística dizem que a radiação tem como equação de estado $\omega=1 / 3$, de forma que a evolução da densidade de energia e do fator de escala durante a época dominada pela radiação é dada por $\rho \propto a^{-4}$ e $a \propto\left(t-t_{i}\right)^{1 / 2}$, respectivamente. A matéria não-relativística corresponde ao caso onde a pressão do fluido pode ser desprezada quando comparada a densidade de energia, isto é, $\omega \simeq 0$. Nesse caso, a evolução da densidade de energia e do fator de escala durante a era dominada pela matéria é dada por $\rho \propto a^{-3}$ e $a \propto\left(t-t_{i}\right)^{2 / 3}$, respectivamente.

Para termos uma aceleração cósmica devemos ter $\ddot{a}>0$ na equação (2.17), o que implica em 


$$
P<-\rho / 3 \Rightarrow \omega<-1 / 3
$$

onde assumimos que $\rho$ é uma quantidade positiva.

Quando $\omega=-1$, isto é, $P=-\rho$, temos da equação (2.18) que $\rho$ é constante. Esse caso corresponde ao caso da constante cosmológica. Como $H$, no universo plano, é então constante, o fator de escala evolui exponencialmente, $a \propto \exp (H t)$.

\subsection{Lei de Hubble}

Nos anos 20 do século XX, Slipher e Hubble observaram que o comprimento de onda observado, $\lambda_{0}$, das linhas de absorção de galáxias distantes é maior que o comprimento de onda observado no referencial em repouso, $\lambda[8]$. Esse desvio para o vermelho é devido ao fato de que o comprimento de onda é proporcional ao fator de escala do universo em expansão. Para quantificar esse efeito, introduzimos o redshift

$$
z \equiv \frac{\lambda_{0}}{\lambda}-1=\frac{a_{0}}{a}-1
$$

onde a presente época corresponde a $z=0$. No restante deste trabalho, tomaremos, sem perda de generalidade, $a_{0}=1$. Ao voltarmos ao passado, $z$ torna-se progressivamente maior. Quando a velocidade de recessão $v$ do objeto é muito menor que a velocidade da luz $c$, temos que $\lambda_{0} \simeq(1+v / c) \lambda$ devido ao efeito Doppler, de forma que

$$
z \simeq v / c
$$

Em um universo em expansão, a distância física $\mathbf{r}$ de um observador posicionado na origem do nosso sistema de referência até um objeto qualquer é dado por $\mathbf{r}=$ $a(t) \mathbf{x}$, onde $\mathbf{x}$ denota a distância comóvel. Para objetos movendo-se com o fluxo de Hubble, a distância comóvel mantém-se constante. Tomando a derivada da equação $\mathbf{r}=a(t) \mathbf{x}$ com respeito a $t$, temos que

$$
\dot{\mathbf{r}}=H \mathbf{r}+a \dot{\mathbf{x}}
$$

A velocidade $\mathbf{v}_{H} \equiv H \mathbf{r}$ aparece por causa da presença da expansão cósmica. Por outro lado, a velocidade $\mathbf{v}_{p} \equiv a \dot{\mathbf{x}}$, chamada de velocidade peculiar, descreve o movimento de um objeto com respeito ao fluxo de Hubble local. A velocidade de um objeto ao longo da direção da linha entre o observador e o objeto é dada por 


$$
v \equiv \dot{\mathbf{r}} \cdot \mathbf{r} / r=H r+\mathbf{v}_{p} \cdot \mathbf{r} / r
$$

onde $r \equiv|\mathbf{r}|$.

Na maioria dos casos, a velocidade peculiar das galáxias não ultrapassa $10^{6} \mathrm{~m} / \mathrm{s}$ [8]. Nessas condições, o termo $\mathbf{v}_{p} \cdot \mathbf{r} / r$ é desprezível em relação ao termo $H r$, de forma que obtemos então

$$
v \simeq H_{0} r
$$

onde substituímos $H$ pelo valor presente $H_{0}$, o que é justificado para pequenos redshifts $(z \ll 1)$.

A constante de Hubble $H_{0}$ é usualmente escrita como

$$
H_{0}=100 h \mathrm{~km} \mathrm{~s}^{-1} \mathrm{Mpc}^{-1}
$$

onde

$$
1 \mathrm{Mpc}=3,08568 \times 10^{24} \mathrm{~cm}=3,26156 \times 10^{6} \text { anos }- \text { luz } .
$$

O parâmetro $h$ descreve a incerteza no valor de $H_{0}$. As observações feitas pelo Hubble Key Project para determinar o parâmetro de Hubble no universo vizinho a nossa galáxia limitam esse valor a [9]

$$
h=0,72 \pm 0,08
$$

\subsection{Componentes que Compõem a Matéria do Uni- verso}

Nesta seção consideraremos os diversos componentes que compõe a matéria do universo. Em geral, a matéria normal pode ser classificada como matéria relativística ou não-relativística. Além da matéria normal, também temos que considerar a energia escura, mas postergaremos sua análise até o Capítulo 4. Outro tipo de matéria, provavelmente um campo escalar, que é conhecido como inflaton, dominou a composição do cosmos durante a época inflacionária do universo, mas para nosso trabalho essa época não será de interesse e, portanto, não discutiremos o caso do inflaton nesta seção.

Primeiramente, definiremos alguns conceitos termodinâmicos comuns à matéria em equilíbrio em um universo homogêneo e isotrópico. Consideraremos uma partí- 
cula com momento $\mathbf{p}$ e massa $m$. A energia da partícula é $E=\sqrt{p^{2}+m^{2}}$, onde $p \equiv|\mathbf{p}|$. A função de distribuição dos momentos em uma temperatura $T$ é dada por [10]

$$
f(\mathbf{p})=\frac{1}{\exp [(E-\mu) / T] \pm 1},
$$

onde $\mu$ é o potencial químico de cada componente material. O sinal positivo representa uma distribuição de Fermi-Dirac, enquanto o negativo representa uma distribuição de Bose-Einstein. Essa função de distribuição é independente da posição x por causa da homogeneidade do universo. A densidade de energia $\rho$ e a pressão $P$ para uma partícula com $g_{*}$ graus de liberdade é dada por

$$
\begin{gathered}
\rho=g_{*} \int \frac{\mathrm{d}^{3} p}{(2 \pi \hbar)^{3}} E(p) f(p)=\frac{g_{*}}{2 \pi} \int_{m}^{\infty} \mathrm{d} E \frac{\left(E^{2}-m^{2}\right)^{1 / 2}}{\exp [(E-\mu) / T] \pm 1} E^{2}, \\
P=g_{*} \int \frac{\mathrm{d}^{3} p}{(2 \pi \hbar)^{3}} \frac{p v}{3} f(p)=g_{*} \int \frac{\mathrm{d}^{3} p}{(2 \pi \hbar)^{3}} \frac{p^{2}}{3 E} f(p) \\
=\frac{g_{*}}{6 \pi^{2}} \int_{m}^{\infty} \mathrm{d} E \frac{\left(E^{2}-m^{2}\right)^{3 / 2}}{\exp [(E-\mu) / T] \pm 1} .
\end{gathered}
$$

Nas expressões finais de (2.35) e (2.36), adotamos unidades de medida em que $\hbar=1$. Agora, trataremos separadamente as partículas relativísticas e não-relativísticas.

\subsubsection{Componentes Relativísticos}

O limite relativístico corresponde a $T \gg m$. Assim, podemos tomar $m \rightarrow 0$ nas equações $(2.35)$ e (2.36). Para partículas não-degeneradas $(T \gg \mu)$, temos que

$$
\begin{aligned}
& \rho= \begin{cases}\left(\pi^{2} / 30\right) g_{*} T^{4}, & \text { (Bosons) } \\
(7 / 8)\left(\pi^{2} / 30\right) g_{*} T^{4}, & \text { (Fermions) }\end{cases} \\
& P=\rho / 3 .
\end{aligned}
$$

O resultado (2.38) mostra que a equação de estado de partículas relativísticas nãodegeneradas é dada, como dissemos anteriormente, por $\omega=1 / 3$.

Os fótons são componentes bosônicos com um potencial químico muito pequeno; o potencial químico da radiação cósmica de fundo, por exemplo, é muito menor que a temperatura $T$ da radiação cósmica de fundo $\left(\mu / T<9 \times 10^{-5}\right)$ [8]. Como o fóton 
possui dois estados de $\operatorname{spin}\left(g_{*}=2\right)$, sua densidade de energia é

$$
\rho_{\gamma}=\frac{\pi^{2}}{15} T^{4}
$$

A presente temperatura dos fótons da radiação cósmica de fundo é medida como sendo $T=2,725 \pm 0,002 \mathrm{~K}[8]$. Usando a conversão $1 \mathrm{~K}^{4}=1,279 \times 10^{-35} \mathrm{~g} \mathrm{~cm}^{-3}$, a densidade dos fótons da radiação cósmica de fundo no presente universo é $\rho_{\gamma}^{(0)}=$ $4,641 \times 10^{-34} \mathrm{~g} \mathrm{~cm}^{-3}$. Isso corresponde a um parâmetro de densidade

$$
\Omega_{\gamma}^{(0)} \equiv \frac{8 \pi G \rho_{\gamma}^{(0)}}{3 H_{0}^{2}}=\frac{\rho_{\gamma}^{(0)}}{\rho_{c}^{(0)}}=2,469 \times 10^{-5} h^{-2} .
$$

Como a densidade de energia $\rho_{\gamma}$ evolui como $\rho_{\gamma} \propto a^{-4}$, vemos através da equação (2.39) que a temperatura da radiação, e também a temperatura do universo, que em número de partículas é dominado por fótons e, portanto, tem sua temperatura em larga-escala determinada pela temperatura da radiação, evolui como $T \propto 1 / a$.

Como as massas dos neutrinos são pequenas, eles comportam-se como partículas relativísticas. Eles são partículas fermiônicas com potencial químico pequeno em relação à temperatura. No modelo padrão da física de partículas, eles são de três tipos. Cada neutrino possui um grau de liberdade de spin e uma antipartícula associada. Usando a equação (2.37) do caso fermiônico, a densidade dos neutrinos, incluindo suas antipartículas, é dada por

$$
\rho_{\nu}=N_{\mathrm{ef}} \frac{7 \pi^{2}}{120} T_{\nu}^{4}
$$

onde $N_{\text {ef }}$ é o número efetivo de espécies de neutrino (3 no modelo padrão) e $T_{\nu}$ é a temperatura dos neutrinos.

A nucleossíntese do Big Bang (NBB) ocorreu ao redor da escala de energia de $\sim 0.1 \mathrm{MeV}$ e formou elementos leves como o deutério e o hélio. O desacoplamento dos neutrinos do restante do plasma cósmico ocorreu imediatamente depois da aniquilação dos elétrons e pósitrons e antes da época da NBB. A presença de graus de liberdade relativísticos extras para o neutrino muda a quantidade prevista para os elementos leves produzidos na NBB, o que permite que tenhamos um limite para $N_{\text {ef }}$. O valor padrão é $N_{\text {ef }}=3,04$ [11], que é ligeiramente maior que 3 devido ao fato dos neutrinos não estarem totalmente desacoplados do plasma cósmico na época da aniquilação dos elétrons e pósitrons. A temperatura dos neutrinos, $T_{\nu}$, é aproximadamente relacionada com a temperatura dos fótons $T_{\gamma}$ através da relação $T_{\nu} / T_{\gamma}=(4 / 11)^{3}$. Essa relação é oriunda da conservação da entropia antes e depois da aniquilação dos elétrons e pósitrons [8]. Das equações (2.39) e (2.41), a relação 
entre a densidade dos neutrinos e dos fótons é $\rho_{\nu}=N_{\text {ef }}(7 / 8)(4 / 11)^{4 / 3} \rho_{\gamma}$. Assim, o parâmetro de densidade da radiação no tempo presente é

$$
\Omega_{r}^{(0)}=\frac{\rho_{\gamma}^{(0)}+\rho_{\nu}^{(0)}}{\rho_{c}^{(0)}}=\Omega_{\gamma}^{(0)}\left(1+0,02271 N_{\mathrm{ef}}\right) .
$$

\subsubsection{Componentes Não-Relativísticos}

No caso de partículas não-relativísticas $(T \ll m)$, as equações $(2.35)$ e $(2.36)$ reduzem-se, no caso em que o sistema está equilíbrio com temperatura $T$, a

$$
\begin{gathered}
\rho=g_{*} m\left(\frac{m T}{2 \pi}\right)^{3 / 2} \exp [-(m-\mu) / T], \\
P=g_{*} T\left(\frac{m T}{2 \pi}\right)^{3 / 2} \exp [-(m-\mu) / T]=\frac{T}{m} \rho .
\end{gathered}
$$

Essas expressões são válidas tanto para partículas bosônicas quanto fermiônicas. A equação (2.44) mostra que a pressão $P$ é suprimida, em relação à densidade de energia $\rho$, pelo fator $T / m \ll 1$. Portanto, a equação de estado para a matéria nãorelativística é $\omega \simeq 0$. O resultado acima mostra que a densidade de energia $\rho$ não é descrita por uma função que depende somente da temperatura $T$, como no caso da radiação, mas que depende também da massa $m$ e o potencial químico $\mu$ das partículas. Portanto, precisamos medir a densidade de partículas não-relativísticas diretamente através de observações astrofísicas.

Durante a época da NBB, os elementos leves como o deutério e o hélio foram formados pelos nêutrons e prótons livres presentes no plasma cósmico. Antes da formação de deutério, a maioria dos nêutrons decaiu em prótons através do decaimento beta, enquanto os nêutrons que não decaíram em prótons eventualmente formaram os núcleos de hélio. Se aumentarmos a densidade de bárions, o processo de nucleossíntese ocorre mais rápido e, portanto, mais nêutrons permanecem sem decair em prótons. Isso leva a um aumento na abundância de hélio e a uma diminuição na abundância de deutério. Assim, a quantidade de elementos leves produzidos durante a época da NBB é sensível à densidade bariônica. A abundância de deutério é obtida ao observarmos as linhas de absorção de quasares localizados em altos redshifts. Desse modo, o parâmetro de densidade dos bárions pode ser estimado como sendo dado por [12]

$$
\Omega_{b}^{(0)} h^{2}=0,020 \pm 0,002 .
$$

As observações das anisotropias da radiação cósmica de fundo também limitam o 
parâmetro de densidade $\Omega_{b}^{(0)}$. Se aumentarmos a densidade de bárions $\rho_{b}$, isso implica em uma velocidade do som $c_{s}$ menor para o fluido de bárions e de fótons. De forma simplificada, a amplitude das anisotropias da radiação cósmica de fundo com número de onda comóvel $k$ satisfaz a equação do oscilador harmônico, $\frac{\mathrm{d}^{2} x}{\mathrm{~d} \eta^{2}}+k^{2} c_{s}^{2} x=0[8,13]$. Para uma velocidade do som pequena, a frequência $k c_{s}$ decresce, de forma que a altura do primeiro pico acústico torna-se maior (isso é devido ao fato que a amplitude do oscilador harmônico torna-se maior com uma constante de mola menor). A densidade de bárions pode ser limitada usando essa propriedade. Dos dados do WMAP combinados com observações de supernovas do tipo Ia (SN Ia) e de oscilações acústicas de bárions (BAO), o parâmetro de densidade presente dos bárions é dado por [7]

$$
\Omega_{b}^{(0)} h^{2}=0,02267 \pm 0,00060 .
$$

Se tomarmos o valor de $h=0,72$, temos que $\Omega_{b}^{(0)}=0,0437$, utilizando o valor central de (2.46).

Como esse parâmetro de densidade é bem menor do que o obtido para o parâmetro de densidade da matéria não-relativística total do universo, vemos que as observações cosmológicas fazem a existência da matéria escura necessária. A existência da matéria escura é também indicada por observações astrofísicas, como a curva de rotação de galáxias e estudos de lentes gravitacionais. Como a matéria escura interage muito fracamente com as partículas do modelo padrão, sua existência só pode ser estudada através de seus efeitos gravitacionais na matéria visível. Por motivo de completeza, temos que distinguir aqui os dois tipos de matéria escura postulados na literatura e definir o que por nós será chamado de matéria escura. Se a matéria escura era não-relativística na época em que se desacoplou dos fótons, ela é chamada de matéria escura fria. Alternativamente, se era relativística, ela é chamada de matéria escura quente, sendo o neutrino seu principal representante. O atual cenário para a formação de estruturas é baseado na aglomeração de matéria escura fria, com as pequenas estruturas formando-se primeiro. Assim, matéria escura fria será sinônima de matéria escura durante o restante deste trabalho.

Os dados das anisotropias da radiação cósmica de fundo mostram que a abundância de matéria escura é cinco vezes maior que a dos bárions. Os dados do WMAP, por exemplo, limitam o parâmetro de densidade da matéria escura em [7]

$$
\Omega_{c}^{(0)} h^{2}=0,11310 \pm 0,00034
$$

Para o valor $h=0,72$, temos que $\Omega_{c}^{(0)}=0,2182$, utilizando o valor central em (2.47). 
A origem e as propriedades fundamentais da matéria escura ainda são objetos de intensa pesquisa teórica e observacional. Existem basicamente dois tipos de candidatos para a matéria escura: os candidatos astrofísicos e os candidatos de partículas elementares. Os candidatos astrofísicos são buracos negros, estrelas de nêutrons e anãs brancas. Contudo, como essas estruturas originam-se de bárions, elas não conseguem explicar toda a abundância da matéria escura no universo. Por exemplo, as observações de microlentes gravitacionais mostram que suas abundâncias não são suficientes para explicar a quantidade de matéria escura que é indicada pelas observações das anisotropias da radiação cósmica de fundo [14].

Os candidatos de partículas elementares para a matéria escura não são previstos no Modelo Padrão da física de partículas. Alguns exemplos desses candidatos são os áxions e as partículas massivas fracamente interagentes (WIMPs). Os áxions foram introduzidos como uma solução para o problema de conservação de CP na cromodinâmica quântica [10]. Já as WIMPs são geralmente oriundas de teorias supersimétricas que estendem o modelo padrão da física de partículas. Por exemplo, os neutralinos são formados através dos quatro autoestados do operador de massa como resultado da mistura dos superparceiros do bóson Z, do fóton, e dos Higgs neutros [15]. O mais leve dos quatro neutralinos é também a mais leve das partículas supersimétricas e possui massas típicas de $m_{n}=100 \mathrm{GeV}-\mathrm{TeV}$ [15]. Os neutralinos mais leves acoplam-se com as outras partículas com magnitude característica da interação fraca, o que faz deles ótimos candidatos para a matéria escura, visto que são pouco interagentes.

\subsection{Distâncias Cósmicas}

Para mais adiante podermos discutir as evidências observacionais da energia escura, é importante discutir como distâncias cósmicas são relacionadas com as observações feitas no espaço-tempo de FRW. Definindo $r=\sin \chi(K=+1), r=\chi(K=0) \mathrm{e}$ $r=\sinh \chi(K=-1)$ na equação $(2.2)$, o elemento de linha tridimensional tornase $[10]$

$$
\mathrm{d} \sigma^{2}=\mathrm{d} \chi^{2}+\left(f_{K}(\chi)\right)^{2}\left(\mathrm{~d} \theta^{2}+\sin \theta \mathrm{d} \phi^{2}\right)
$$

onde

$$
f_{K}(\chi)= \begin{cases}\sin \chi, & (K=+1) \\ \chi, & (K=0) \\ \sinh \chi, & (K=-1)\end{cases}
$$


A função (2.49) pode ser escrita de forma unificada como

$$
f_{K}(\chi)=\frac{1}{\sqrt{-K}} \sinh (\sqrt{-K} \chi)
$$

Sendo que o caso do universo plano é recuperado tomando o limite $K \rightarrow-0$.

\subsubsection{Distância Comóvel}

Quando a luz está viajando através da direção $\chi$, isto é, sem componente transversal, ela satisfaz a equação da geodésica: $\mathrm{d} s^{2}=-c^{2} \mathrm{~d} t^{2}+a^{2}(t) \mathrm{d} \chi^{2}=0$. Consideraremos o caso em que a luz emitida no tempo $t=t_{1} \operatorname{com} \chi=\chi_{1}$ (redshift $z$ ) chega no observador no tempo $t=t_{0} \operatorname{com} \chi=0$ (correspondendo a $z=0$ ). Nesse caso, integrando a equação $\mathrm{d} \chi=-c \mathrm{~d} t / a(t)$, a distância comóvel será dada por

$$
d_{c} \equiv \chi_{1}=\int_{0}^{\chi_{1}} \mathrm{~d} \chi=-\int_{t_{0}}^{t_{1}} \frac{c}{a(t)} \mathrm{d} t .
$$

Da equação (2.26), temos que $\mathrm{d} t=\mathrm{d} z /[H(z+1)]$. Assim, a distância comóvel pode ser escrita como

$$
d_{c}=\frac{c}{a_{0} H_{0}} \int_{0}^{z} \frac{\mathrm{d} z}{E(z)}
$$

onde

$$
E(z) \equiv H(z) / H_{0}
$$

A função $\int_{0}^{z} \mathrm{~d} z / E(z)$ pode ser expandida em uma série de Taylor ao redor de $z=0$ $[10]$,

$$
\int_{0}^{z} \frac{\mathrm{d} z}{E(z)}=z-\frac{E^{\prime}(0)}{2} z^{2}+\frac{1}{6}\left[2 E^{\prime}(0)^{2}-E^{\prime \prime}(0)\right] z^{3}+\mathcal{O}\left(z^{4}\right)
$$

onde a linha representa uma derivada com respeito a $z$. Para redshifts $z$ muito menores que a unidade, a distância comóvel é dada aproximadamente por

$$
d_{c} \simeq \frac{c}{a_{0} H_{0}} z .
$$

Usando a relação (2.27), temos que

$$
v \simeq\left(a_{0} H_{0}\right) d_{c}
$$

Para a distância física $r=a_{0} d_{c}$, temos que $r \simeq\left(c / H_{0}\right) z \simeq v / H_{0}$, o que mostra que a lei de Hubble linear é satisfeita para pequenos redshifts. 


\subsubsection{Distância de Luminosidade}

A distância de luminosidade é definida como [10]

$$
d_{L}^{2} \equiv \frac{L_{s}}{4 \pi \mathcal{F}}
$$

onde $L_{s}$ é a luminosidade absoluta da fonte e $\mathcal{F}$ é o fluxo observado. A luminosidade observada $L_{0}$ (detectada em $\chi=0$ e $z=0$ ) é diferente da luminosidade absoluta $L_{s}$ da fonte (emitida na distância comóvel $\chi$ com redshift $z$ ). O fluxo $\mathcal{F}$ é definido por $\mathcal{F}=L_{0} / S$, onde $S=4 \pi\left(a_{0} f_{K}(\chi)\right)^{2}$ é a área da esfera com centro na fonte e com raio estendendo-se até $z=0$. Dessa maneira, a distância de luminosidade (2.57) torna-se

$$
d_{L}^{2}=\left(a_{0} f_{K}(\chi)\right)^{2} \frac{L_{s}}{L_{0}}
$$

Se tomarmos a energia da luz emitida no intervalo $\Delta t_{1}$ como $\Delta E_{1}$, a luminosidade absoluta é definida por $L_{s}=\Delta E_{1} / \Delta t_{1}$. Similarmente, a luminosidade observada é dada por $L_{0}=\Delta E_{0} / \Delta t_{0}$, onde $\Delta E_{0}$ é a energia da luz medida no intervalo $\Delta t_{0}$. Como a energia de um fóton é inversamente proporcional ao seu comprimento de onda $\lambda$, temos que $\Delta E_{1} / \Delta E_{0}=\lambda_{0} / \lambda_{1}=1+z$. Como $c=\lambda / \Delta t$ é constante, temos que $\lambda_{1} / \Delta t_{1}=\lambda_{0} / \Delta t_{0}$, onde $\lambda_{1}$ e $\lambda_{0}$ são os comprimentos de onda da luz no ponto de emissão e de observação, respectivamente. Isso leva a relação $\Delta t_{0} / \Delta t_{1}=\lambda_{0} / \lambda_{1}=$ $1+z$. Portanto, temos que

$$
\frac{L_{s}}{L_{0}}=\frac{\Delta E_{1}}{\Delta E_{0}} \frac{\Delta t_{0}}{\Delta t_{1}}=(1+z)^{2} .
$$

Das equações (2.58) e (2.59), a distância de luminosidade torna-se

$$
d_{L}=a_{0} f_{K}(\chi)(1+z)
$$

Utilizando a equação (2.50) para $f_{K}(\chi)$ e com a distância comóvel dada por (2.52), temos que a distância de luminosidade é dada por

$$
d_{L}=\frac{c(1+z)}{H_{0} \sqrt{\Omega_{K}^{(0)}}} \sinh \left(\sqrt{\Omega_{K}^{(0)}} \int_{0}^{z} \frac{\mathrm{d} z}{E(z)}\right)
$$

onde $\Omega_{K}^{(0)}=-K c^{2} /\left(a_{0} H_{0}\right)^{2}$. 


\subsubsection{Distância do Diâmetro Angular}

A distância do diâmetro angular $d_{A}$ é definida, com base na geometria euclidiana, por [10]

$$
d_{A} \equiv \frac{\Delta x}{\Delta \theta},
$$

onde $\Delta \theta$ é o ângulo que um objeto de tamanho $\Delta x$ projetado ortogonalmente a linha de visão do observador estende-se.

Como a fonte fica na superfície de uma esfera com raio $\chi$ que tem o observador posicionado no centro, temos que o tamanho $\Delta x$, no tempo $t_{1}$, no espaço de FRW com elemento de linha tridimensional dado pela equação (2.48), é dado por

$$
\Delta x=a\left(t_{1}\right) f_{K}(\chi) \Delta \theta
$$

Dessa maneira, a distância do diâmetro angular é

$$
d_{A}=a\left(t_{1}\right) f_{K}(\chi)=\frac{a_{0} f_{K}(\chi)}{1+z}=\frac{1}{1+z} \frac{c}{H_{0} \sqrt{\Omega_{K}^{(0)}}} \sinh \left(\sqrt{\Omega_{K}^{(0)}} \int_{0}^{z} \frac{\mathrm{d} z}{E(z)}\right)
$$

Comparando a equação (2.64) com a equação (2.57), temos a relação

$$
d_{A}=\frac{d_{L}}{(1+z)^{2}}
$$

Essa relação é válida em qualquer métrica, ou mesmo em qualquer teoria da gravitação baseada na geometria do espaço-tempo, desde que o fluxo de energia seja conservado.

\subsection{A Equação de Estado da Energia Escura}

Um dos parâmetros mais importantes a ser determinado pelas futuras observações cosmológicas é a equação de estado da energia escura, $\omega$. Essa equação de estado depende do modelo para a energia escura. Para a constante cosmológica, ela é dada por $\omega=-1$. Para outros modelos, ela é uma função livre que varia com o tempo. Como o estudo de sua evolução com o tempo é fundamental para que possamos discernir qual é o melhor modelo para energia escura, discutiremos como a determinação dessa evolução poderá ser feita no futuro. Para isso, consideraremos o universo como sendo composto por radiação, matéria não-relativística e energia escura. Como as densidades de energia da radiação e da matéria não-relativística, $\rho_{r}$ 
e $\rho_{m}$, respectivamente, evoluem como $\rho_{r} \propto a^{-4}$ e $\rho_{m} \propto a^{-3}$, elas podem ser expressas na forma parametrizada

$$
\begin{gathered}
\rho_{r}=\rho_{r}^{(0)}\left(a_{0} / a\right)^{4}=\rho_{r}^{(0)}(1+z)^{4}, \\
\rho_{m}=\rho_{m}^{(0)}\left(a_{0} / a\right)^{3}=\rho_{m}^{(0)}(1+z)^{3} .
\end{gathered}
$$

O redshift que corresponde a igualdade entre as densidades da matéria e da radiação é dado por

$$
1+z_{\mathrm{eq}}=\frac{\rho_{m}^{(0)}}{\rho_{r}^{(0)}}=\frac{\Omega_{m}^{(0)}}{\Omega_{r}^{(0)}},
$$

onde $\Omega_{r}^{(0)}$ é dado pela equação (2.42) juntamente com a equação (2.40). O parâmetro de densidade $\Omega_{m}^{(0)}$ é a soma da contribuição de bárions e da matéria escura, $\Omega_{m}^{(0)}=$ $\Omega_{b}^{(0)}+\Omega_{c}^{(0)}$.

Para um número efetivo de neutrinos $N_{\mathrm{ef}}=3,04$, temos que [8]

$$
1+z_{\mathrm{eq}}=2,396 \times 10^{4} \Omega_{m}^{(0)} h^{2} .
$$

A energia escura, com uma equação de estado $\omega_{d}=P_{d} / \rho_{d}$, satisfaz a equação de continuidade

$$
\dot{\rho}_{d}+3 H\left(\rho_{d}+P_{d}\right)=0 .
$$

Integrando essa equação e utilizando a relação $\mathrm{d} t=-\mathrm{d} z /[H(1+z)]$, temos que [16]

$$
\rho_{d}=\rho_{d}^{(0)} \exp \left[\int_{0}^{z} \frac{3\left(1+\omega_{d}\right)}{1+z} \mathrm{~d} z\right]
$$

Da equação de Friedmann (2.15), temos que

$$
H^{2}=\frac{8 \pi G}{3}\left(\rho_{r}+\rho_{m}+\rho_{d}\right)-\frac{K}{a^{2}} .
$$

Da equação (2.72), vemos que os parâmetros de densidade atuais, definidos na equação (2.22), obedecem a relação

$$
\Omega_{r}^{(0)}+\Omega_{m}^{(0)}+\Omega_{d}^{(0)}+\Omega_{K}^{(0)}=1 .
$$

Desse modo, a equação (2.72) pode ser escrita na forma 


$$
\begin{aligned}
H^{2}(z) & =H_{0}^{2}\left[\Omega_{r}^{(0)}(1+z)^{4}+\Omega_{m}^{(0)}(1+z)^{3}\right. \\
& \left.+\Omega_{d}^{(0)} \exp \left(\int_{0}^{z} \frac{3\left(1+\omega_{d}\right)}{1+z} \mathrm{~d} z\right)+\Omega_{K}^{(0)}(1+z)^{2}\right] .
\end{aligned}
$$

Derivando essa equação com respeito a $z$, achamos que a equação de estado da energia escura pode ser expressa como [16]

$$
\omega_{d}(z)=\frac{(1+z)\left(E^{2}(z)\right)^{\prime}-3 E^{2}(z)-\Omega_{r}^{(0)}(1+z)^{4}+\Omega_{K}^{(0)}(1+z)^{2}}{3\left[E^{2}(z)-\Omega_{r}^{(0)}(1+z)^{4}-\Omega_{m}^{(0)}(1+z)^{3}-\Omega_{K}^{(0)}(1+z)^{4}\right]},
$$

onde $E(z)$ foi definido na equação $(2.53)$ e a linha representa uma derivada em relação a $z$. Através da equação (2.61), a quantidade $E(z)$ pode ser escrita em termos de $d_{L}$

$$
E^{2}(z)=\frac{(1+z)^{2}\left[c^{2}(1+z)^{2}+\Omega_{K}^{(0)} H_{0}^{2} d_{L}(z)^{2}\right]}{\left[(1+z) H_{0} d_{L}^{\prime}(z)-H_{0} d_{L}(z)\right]^{2}} .
$$

Para um universo plano essa relação reduz-se a

$$
E(z)=\frac{c}{H_{0}}\left[\frac{\mathrm{d}}{\mathrm{d} z}\left(\frac{d_{L}(z)}{1+z}\right)\right]^{-1} .
$$

Portanto, podemos ver que se a distância de luminosidade $d_{L}(z)$ for medida observacionalmente, podemos determinar a evolução de $E(z)$ através da equação (2.76) e então obter $\omega_{d}(z)$ através da equação (2.75). Todavia, para conseguirmos isso, a determinação da distância de luminosidade precisa tornar-se muito mais precisa e confiável do que conseguimos obter atualmente com observações de supernovas do tipo Ia, por exemplo. 



\section{Capítulo 3}

\section{Teoria de Perturbação}

No capítulo anterior desta dissertação, o universo foi tratado como sendo isotrópico e homogêneo em larga escala, com o campo gravitacional descrito pela métrica de FRW. Isso obviamente é somente uma aproximação que ignora a maioria das estruturas presentes no universo, como os aglomerados de galáxias e as estrelas. Neste capítulo, trabalharemos com uma métrica que descreve um campo gravitacional quase homogêneo e isotrópico. Como sabemos que as inomogeneidades na distribuição da matéria do universo foram, por boa parte de sua história, muito pequenas, precisaremos somente perturbar linearmente a métrica de FRW e estudar as equações de Einstein em primeira ordem. Com esse formalismo, poderemos estudar as anisotropias da radiação cósmica de fundo e a distribuição da matéria não-relativística no universo.

\subsection{Perturbações Lineares na Relatividade Geral}

Uma métrica que é sutilmente distinta da métrica que descreve o espaço-tempo de FRW pode ser escrita como a soma da métrica não-perturbada de FRW e mais alguma outra contribuição que podemos chamar de métrica perturbada. Se a parte perturbada for considerada pequena, então essa divisão da métrica total em uma parte de fundo e uma parte perturbada nos levará a resultados extremamente úteis, que, do ponto de vista histórico, revolucionaram a Cosmologia.

Para considerarmos as equações relativísticas perturbadas, devemos primeiramente perturbar a métrica, que em primeira ordem em teoria de perturbação é dada por

$$
g_{\mu \nu}=\bar{g}_{\mu \nu}+\delta g_{\mu \nu}
$$


onde todas as componentes da métrica perturbada $\delta g_{\mu \nu}$ devem ser pequenas com respeito a métrica de fundo $\bar{g}_{\mu \nu}$. Neste capítulo, escreveremos a métrica diretamente em termos do tempo conforme $\eta=\int a^{-1} \mathrm{~d} t$. Consideraremos as perturbações cosmológicas sobre a métrica de FRW plana, que é dada por

$$
\mathrm{d} s^{2}=\bar{g}_{\mu \nu} \mathrm{d} x^{\mu} \mathrm{d} x^{\nu}=a^{2}\left(-\mathrm{d} \eta^{2}+\delta_{i j} \mathrm{~d} x^{i} \mathrm{~d} x^{j}\right) .
$$

Também utilizaremos o parâmetro de Hubble conforme,

$$
\mathcal{H} \equiv \frac{1}{a} \frac{\mathrm{d} a}{\mathrm{~d} \eta}=H a
$$

Na Relatividade Geral, as equações de campo são invariantes sob uma mudança de coordenada geral. Isso significa que a divisão entre uma métrica de fundo e uma métrica perturbada não é dada univocamente. Gostaríamos, entretanto, de manter a métrica de FRW como a métrica de fundo toda vez que fizermos uma transformação de coordenadas geral. Para isso, podemos selecionar uma classe de transformações infinitesimais que deixa $\bar{g}_{\mu \nu}$ a mesma, enquanto a métrica perturbada $\delta g_{\mu \nu}$ é sujeita a uma transformação. Essa classe de transformações é chamada de transformações de gauge.

Embora uma transformação de gauge seja expressa como uma mudança de coordenadas ordinária, é importante destacar que, ao contrário de transformações de coordenadas ordinárias, elas não conectam diferentes observadores no mesmo espaçotempo, mas sim dois espaços-tempos distintos vistos pelo mesmo observador. Essa é a razão do porquê de quantidades escalares transformarem-se sob transformações de gauge, mas não sob uma transformação de coordenadas geral [17].

\subsection{Gauge Newtoniano}

Neste capítulo, consideraremos somente o gauge newtoniano, visto que as equações e a interpretação física delas são mais compatíveis com nossos presentes propósitos. A métrica perturbada mais geral pode ser escrita como $g_{\mu \nu}=\bar{g}_{\mu \nu}+\delta g_{\mu \nu}$, onde a parte perturbada é dada por $[8,18]$

$$
\delta g_{\mu \nu}=a^{2}\left(\begin{array}{cc}
-2 \Psi & w_{i} \\
w_{i} & 2 \Phi \delta_{i j}+h_{i j}
\end{array}\right),
$$

onde $\Psi$ e $\Phi$ são escalares espaciais, $w_{i}$ é um vetor espacial, e $h_{i j}$ é um tensor de traço zero. Toda quantidade representando uma perturbação $(\Psi, \Phi$, etc.) depende do espaço e do tempo, mas omitiremos essa dependência aqui por simplificação. Essa 
divisão da métrica em termos escalares, vetoriais e tensoriais é totalmente geral. Por exemplo, é fácil de mostrar que $g_{00}$ é um escalar espacial. Se $\tilde{x}^{\mu}=f\left(x^{\mu}\right)$ for uma transformação geral, o tensor métrico transforma-se como [6]

$$
\tilde{g}_{\mu \nu}=\frac{\partial x^{\alpha}}{\partial \tilde{x}^{\mu}} \frac{\partial x^{\beta}}{\partial \tilde{x}^{\nu}} g_{\alpha \beta}
$$

Se realizarmos uma transformação puramente espacial, $\tilde{x}^{0}=x^{0}, \tilde{x}^{i}=f\left(x^{i}\right)$, temos imediatamente que $\tilde{g}_{00}=g_{00}$, como é exigido para um escalar espacial. Exemplos similares mostram que $g_{0 i}$ comporta-se como um vetor espacial e $g_{i j}$ como um tensor espacial.

Se escrevermos a métrica perturbada como $g_{\mu \nu}=\bar{g}_{\mu \nu}+\delta g_{\mu \nu}$, a condição $g_{\alpha \gamma} g^{\gamma \beta}=$ $\delta_{\alpha}^{\beta}$ impõe a seguinte relação, em primeira ordem, para a métrica perturbada,

$$
\delta g^{\mu \nu}=-\delta g_{\alpha \beta} \bar{g}^{\alpha \mu} \bar{g}^{\beta \nu}
$$

Uma decomposição análoga à do $g_{\mu \nu}$ pode ser feita para qualquer tensor de rank dois, como, por exemplo, o tensor de energia-momento. Utilizando o teorema de Helmholtz [19] podemos decompor o vetor $w_{i}$ em uma componente longitudinal e uma transversal,

$$
w_{i}=w_{i}^{\|}+w_{i}^{\perp}
$$

onde por construção

$$
\nabla \cdot w_{i}^{\perp}=\nabla \times w_{i}^{\|}=0 .
$$

O componente longitudinal, $w_{i}^{\|}$, sendo irrotacional, é o gradiente de uma quantidade escalar $w_{s}$, isto é, $w_{i}^{\|}=\nabla_{i} w_{s}$. Quando derivarmos as equações de Einstein para os componentes $(0 i)$, teremos termos longitudinais e transversais, ambos em $G_{0 i}$ e $T_{0 i}$. Tomando o rotacional das equações, temos somente equações transversais. Por outro lado, tomando a divergência, temos somente equações longitudinais. Portanto, os dois componentes desacoplam-se completamente um do outro e evoluem independentemente, de forma que podemos tratá-los separadamente. Como a perturbação da densidade $\delta$ é uma quantidade escalar, somente termos longitudinais, que podem ser derivados de uma quantidade escalar, acoplam-se com perturbações de densidade.

Um argumento similar pode ser utilizado para a parte espacial do tensor de traço zero $h_{i j}$. Esse tensor pode ser escrito, em geral, como uma soma de três termos de traço zero, 


$$
h_{i j}=h_{i j}^{\|}+h_{i j}^{\perp}+h_{i j}^{T},
$$

onde as divergências $\partial^{i} h_{i j}^{\|}$e $\partial^{i} h_{i j}^{\perp}$ (que são vetores) são do tipo longitudinal e transversal, respectivamente, e $h_{i j}^{T}$ é transverso, isto é,

$$
\epsilon_{l j k} \partial_{i} \partial_{k} h_{i j}^{\|}=0, \quad \partial_{i} \partial_{j} h_{i j}^{\perp}=0, \quad \partial_{i} h_{i j}^{T}=0 .
$$

Aqui o tensor de Levi-Civita, $\epsilon_{i j k}$, é +1 para permutações pares (123), -1 para permutações ímpares e 0 para índices repetidos. Isso significa que o rotacional de $\partial_{i} h_{i j}^{\|}$, assim como as divergências de $\partial_{j} h_{i j}^{\perp}$ e $h_{i j}^{T}$, se anula. Agora, como $\partial_{i} h_{i j}^{\|}$tem rotacional zero, ele pode ser escrito em termos de uma função escalar $E$. É fácil de verificar que $\epsilon_{l j k} \partial_{i} \partial_{k} h_{i j}^{\|}=0$ se tivermos

$$
h_{i j}^{\|}=\left(\partial_{i} \partial_{j}-\frac{1}{3} \delta_{i j} \nabla^{2}\right) E \equiv D_{i j} E .
$$

Por outro lado, as perturbações $h_{i j}^{\perp}$ e $h_{i j}^{T}$ não podem ser derivadas de funções escalares. A primeira perturbação é um vetor dando origem a perturbações rotacionais da velocidade, enquanto a segunda é um tensor que dá origem a ondas gravitacionais. Essas duas perturbações se desacoplam completamente dos termos escalares e podem ser tratadas separadamente. Os termos que são intrinsecamente vetoriais se acoplam com modos puramente rotacionais, enquanto termos tensoriais representam ondas gravitacionais acopladas com a matéria somente através de perturbações anisotrópicas. Esses modos não serão mais considerados neste trabalho por não serem de grande interesse na atual pesquisa da energia escura.

Dessa maneira, utilizaremos somente a parte de $w_{i}$ e $h_{i j}$ oriunda de funções escalares. Isso pode ser feito ao introduzirmos dois novos escalares, $B$ e $E$, que produzem o vetor $B_{, i}$ e o tensor $D_{i j} E$. A métrica perturbada é, então, dada por

$$
\delta g_{\mu \nu}=a^{2}\left(\begin{array}{cc}
-2 \Psi & B_{, i} \\
B_{, i} & 2 \Phi \delta_{i j}+D_{i j} E
\end{array}\right) .
$$

Das quatro funções escalares $\Psi, \Phi, B$ e $E$, podemos construir quantidades que são invariantes de gauge, isto é, combinações que continuam invariantes, em primeira ordem, após uma transformação de coordenadas infinitesimal, $\tilde{x}^{\mu}=x^{\mu}+\xi^{\mu}$. Contudo, a análise da teoria de perturbações é mais simples quando escolhemos um gauge específico. Isso pode ser feito ao impormos quatro condições de gauge para a métrica, o que corresponde às quatro transformações de coordenadas possíveis. Aqui, vamos escolhê-las de forma que $w_{i}=0$, isto é, $B=0$ e $E=0$. Essa escolha finalmente nos dá a métrica perturbada no gauge newtoniano, 


$$
\mathrm{d} s^{2}=a^{2}(\eta)\left[-(1+2 \Psi) \mathrm{d} \eta^{2}+(1+2 \Phi) \delta_{i j} \mathrm{~d} x^{i} \mathrm{~d} x^{j}\right]
$$

Para derivarmos as equações de Einstein em primeira ordem, vamos decompor o tensor de Einstein $G_{\nu}^{\mu}$ e o tensor de energia-momento $T_{\nu}^{\mu}$ em uma parte de fundo e uma perturbada: $G_{\nu}^{\mu}=\bar{G}_{\nu}^{\mu}+\delta G_{\nu}^{\mu}$ e $T_{\nu}^{\mu}=\bar{T}_{\nu}^{\mu}=\delta T_{\nu}^{\mu}$. A evolução do fundo cosmológico é obtida ao resolvermos as equações de Einstein em ordem zero, $\bar{G}_{\nu}^{\mu}=$ $8 \pi G \bar{T}_{\nu}^{\mu}$. As equações de Einstein em primeira ordem, por sua vez, são dadas por

$$
\delta G_{\nu}^{\mu}=8 \pi G \delta T_{\nu}^{\mu}
$$

O lado esquerdo da equação (3.14) pode ser facilmente calculado. Primeiramente, calcularemos os símbolos de Christoffel perturbados $\delta \Gamma_{\mu \lambda}^{\nu}$ através da relação $[10,18]$

$$
\delta \Gamma_{\mu \lambda}^{\nu}=\frac{1}{2} \delta g^{\mu \alpha}\left(g_{\alpha \nu, \lambda}+g_{\alpha \lambda, \nu}-g_{\nu \lambda, \alpha}\right)+\frac{1}{2} g^{\mu \alpha}\left(\delta g_{\alpha \nu, \lambda}+\delta g_{\alpha \lambda, \nu}-\delta g_{\nu \lambda, \alpha}\right) .
$$

Para a métrica (3.13), os componentes diferentes de zero dos símbolos de Christoffel perturbados são

$$
\begin{aligned}
& \delta \Gamma_{i j}^{0}=\delta_{i j}\left[2 \mathcal{H}(\Phi-\Psi)+\Phi^{\prime}\right] \\
& \delta \Gamma_{00}^{0}=\Psi^{\prime} \\
& \delta \Gamma_{0 i}^{0}=\delta \Gamma_{00}^{i}=\Psi_{, i} \\
& \Gamma_{j 0}^{i}=\delta_{j}^{i} \Phi^{\prime}
\end{aligned}
$$

onde a linha representa a derivada com respeito ao tempo conforme.

O próximo passo é derivar as perturbações no tensor de Ricci e no escalar de Ricci,

$$
\begin{gathered}
\delta R_{\mu \nu}=\delta \Gamma_{\mu \nu, \alpha}^{\alpha}-\delta \Gamma_{\mu \alpha, \nu}^{\alpha}+\delta \Gamma_{\mu \nu}^{\alpha} \Gamma_{\alpha \beta}^{\beta}+\Gamma_{\mu \nu}^{\alpha} \delta \Gamma_{\alpha \beta}^{\beta}-\delta \Gamma_{\mu \beta}^{\alpha} \Gamma_{\alpha \nu}^{\beta}-\Gamma_{\mu \beta}^{\alpha} \delta \Gamma_{\alpha \nu}^{\beta} \\
\delta R=\delta g^{\mu \alpha} R_{\mu \alpha}+g^{\mu \alpha} \delta R_{\alpha \mu} .
\end{gathered}
$$

Finalmente, o tensores de Einstein perturbados são dados por

$$
\delta G_{\mu \nu}=\delta R_{\mu \nu}-\frac{1}{2} \delta g_{\mu \nu} R-\frac{1}{2} g_{\mu \nu} \delta R
$$




$$
\delta G_{\nu}^{\mu}=\delta g^{\mu \alpha} G_{\alpha \nu}+g^{\mu \alpha} \delta G_{\alpha \nu}
$$

Para a métrica (3.13), obtemos [10,18]

$$
\begin{aligned}
& \delta G_{0}^{0}=2 a^{-2}\left[3 \mathcal{H}\left(\mathcal{H} \Psi-\Phi^{\prime}\right)+\nabla^{2} \Phi\right] \\
& \delta G_{i}^{0}=2 a^{-2}\left(\Phi^{\prime}-\mathcal{H} \Psi\right)_{\mid i} \\
& \delta G_{j}^{i}=2 a^{-2}\left[\left(\mathcal{H}^{2}+2 \mathcal{H}^{\prime}\right) \Psi+\mathcal{H} \Psi^{\prime}-\Phi^{\prime \prime}-2 \mathcal{H} \Phi^{\prime}\right] \delta_{j}^{i} \\
& +a^{-2}\left[\nabla^{2}(\Psi+\Phi) \delta_{j}^{i}-(\Psi-\Phi)_{\mid j}^{i}\right],
\end{aligned}
$$

onde o índice inferior | representa uma derivada covariante com respeito à métrica espacial e $\nabla^{2} f \equiv f_{; \mu}^{; \mu}$. Quando a fonte da matéria é especificada, o tensor de energiamomento perturbado $\delta T_{\nu}^{\mu}$ pode ser determinado. Dessa maneira, da equação (3.14), obtemos as equações perturbadas lineares para a evolução do universo. O tensor de energia-momento também satisfaz a equação de continuidade $T_{\nu ; \mu}^{\mu}=0$. A parte de primeira ordem dessa equação é

$$
\delta T_{\nu ; \mu}^{\mu}=0
$$

Com o intuito de calcular o tensor de energia-momento, precisamos perturbar a quadrivelocidade $u^{\mu}=\frac{\mathrm{d} x^{\mu}}{\mathrm{d} s}$. Desprezando as perturbações além da primeira ordem, temos que

$$
\begin{aligned}
& u^{\mu}=\left[\frac{1}{a}(1-\Psi), \frac{v^{i}}{a}\right], \\
& u_{\mu}=g_{\mu \nu} u^{\nu}=\left[-a(1+\Psi), a v_{i}\right], \\
& u_{\mu} u^{\mu}=-1,
\end{aligned}
$$

onde $v^{i}=\frac{\mathrm{d} x^{i}}{\mathrm{~d} \eta}=a \frac{\mathrm{d} x^{i}}{\mathrm{~d} t}$ é a velocidade peculiar da matéria com respeito à expansão geral.

\subsection{Modelo Cosmológico com um Único Fluido}

Como nosso primeiro e mais simples exemplo, consideraremos um modelo constituído por um único fluido com um tensor de energia-momento $T_{\mu \nu}$ dado por

$$
T_{\mu \nu}=(\rho+P) u_{\mu} u_{\nu}+P g_{\mu \nu}+\left[q_{\mu} u_{\nu}+q_{\nu} u_{\mu}+\pi_{\mu \nu}\right]
$$


onde $q_{\mu}$ é o vetor de fluxo de calor e $\pi_{\mu \nu}$ é o tensor de estresse anisotrópico. Os termos dentro dos colchetes na equação (3.29) são importantes somente para fluidos com energia interna de tamanho comparável a densidade de energia total do fluido. Para fluidos perfeitos, temos que $q_{\mu}=0$ e $\pi_{\mu \nu}=0$. Nesta seção, vamos somente nos atentar aos fluidos perfeitos. Assumiremos também que o fluido perturbado mantém-se perfeito. Isso implica que $\Sigma_{j}^{i} \equiv \delta T_{j}^{i}=0(i \neq j)$.

A seguir, consideraremos as seguintes grandezas como representantes das quantidades perturbadas,

$$
\delta \equiv \frac{\delta \rho}{\rho}, \quad \theta \equiv \nabla_{i} v^{i}
$$

onde $\delta \rho / \rho \equiv(\rho(x)-\bar{\rho}) / \bar{\rho}$ é o contraste de densidade $(\rho(x)$ é o campo de densidade e $\bar{\rho}$ é a média espacial da densidade) e $\theta$ é a divergência da velocidade.

A partir da equação (3.29), o tensor de energia-momento perturbado para um fluido perfeito com uma equação de estado $\omega=P / \rho$ pode ser escrito como $[10,18]$

$$
\delta T_{\nu}^{\mu}=\rho\left[\delta\left(1+c_{s}^{2}\right) u_{\nu} u^{\mu}+(1+\omega)\left(\delta u_{\nu} u^{\mu}+u_{\nu} \delta u^{\mu}\right)+c_{s}^{2} \delta \delta_{\nu}^{\mu}\right]
$$

Nessa expressão, introduzimos a velocidade do som, $c_{s}^{2} \equiv \delta P / \delta \rho$. Se $P$, mesmo quando perturbado, depender somente de $\rho$, caso de um fluido barotrópico, temos que

$$
c_{s}^{2} \equiv \frac{\delta P}{\delta \rho}=\frac{\mathrm{d} P}{\mathrm{~d} \rho}=\frac{\dot{P}}{\dot{\rho}} .
$$

A última passagem é válida somente na métrica de FRW, onde no nível de fundo todas as grandezas físicas dependem somente do tempo. $c_{s}$ é calculado em primeira ordem, visto que ele sempre aparecerá como um fator composto por variáveis de primeira ordem, afinal, em um fluido totalmente homogêneo e isotrópico, não temos ondas de som propagando-se. Em geral, entretanto, a pressão $P$ pode depender de graus de liberdade internos do fluido, como, por exemplo, da entropia $s$. Assim,

$$
c_{s}^{2}=\frac{\delta P(\rho, s)}{\delta \rho}=\frac{\partial P}{\partial \rho}+\frac{\partial P}{\partial s} \frac{\partial s}{\partial \rho}=c_{s(a)}^{2}+c_{s(n a)}^{2},
$$

onde $c_{s(a)} \equiv \sqrt{\dot{P} / \dot{\rho}}$ é a velocidade do som adiabática e $c_{s(n a)}$ é a velocidade do som não-adiabática. A velocidade do som não-adiabática, em geral, dependerá de propriedades microscópicas do fluido e aparecerá como uma função livre somente no nível perturbativo. Portanto, as equações gravitacionais de primeira ordem são completamente especificadas se, para cada fluido, determinarmos a equação de estado $\omega(a)$ e a velocidade do som total $c_{s}(a)$ ou, equivalentemente, se determinarmos 
para um fluido a função $P(\rho, s)$ que determinará ambos. Contudo, neste capítulo, consideraremos somente fluidos barotrópicos.

Os componentes do tensor de energia-momento são

$$
\begin{aligned}
& \delta T_{0}^{0}=-\delta \rho \\
& \delta T_{i}^{0}=-\delta T_{0}^{i}=(1+\omega) \rho v^{i} \\
& \delta T_{1}^{1}=\delta T_{2}^{2}=\delta T_{3}^{3}=c_{s}^{2} \delta \rho .
\end{aligned}
$$

Dessa forma, as equações perturbadas de Einstein (3.14) nos dão

$$
\begin{aligned}
& 3 \mathcal{H}\left(\mathcal{H} \Psi-\Phi^{\prime}\right)+\nabla^{2} \Phi=-4 \pi G a^{2} \delta \rho \\
& \nabla^{2}\left(\Phi^{\prime}-\mathcal{H} \Psi\right)=4 \pi G a^{2}(1+\omega) \rho \theta \\
& \Psi=-\Phi \\
& \Phi^{\prime \prime}+2 \mathcal{H} \Phi^{\prime}-\mathcal{H} \Psi^{\prime}-\left(\mathcal{H}^{2}+2 \mathcal{H}^{\prime}\right) \Psi=-4 \pi G a^{2} c_{s}^{2} \delta \rho .
\end{aligned}
$$

Note que essas equações são oriundas das componentes (00), $(0 i),(i j)$ e $(i i)$ das equações de Einstein, respectivamente.

Podemos obter outras equações para nossas grandezas perturbadas através da equação de continuidade (3.27). Lembrando que

$$
T_{\nu ; \mu}^{\mu}=T_{\nu, \mu}^{\mu}-\Gamma_{\nu \beta}^{\alpha} T_{\alpha}^{\beta}+\Gamma_{\beta \alpha}^{\alpha} T_{\nu}^{\beta}
$$

temos que a componente $\nu=0$ da equação (3.27) é dada por

$$
\delta T_{0, \mu}^{\mu}-\delta \Gamma_{0 \beta}^{\alpha} T_{\alpha}^{\beta}-\Gamma_{0 \beta}^{\alpha} \delta T_{\alpha}^{\beta}+\delta \Gamma_{\beta \alpha}^{\alpha} T_{0}^{\beta}+\Gamma_{\beta \alpha}^{\alpha} T_{0}^{\beta}=0 .
$$

Essa equação pode ser reescrita como

$$
(\delta \rho)^{\prime}+3 \mathcal{H}(\delta \rho+\delta P)=-(\rho+P)\left(\theta+3 \Phi^{\prime}\right)
$$

onde utilizamos as equações (3.16)-(3.19). Usando a equação de conservação nãoperturbada $\rho^{\prime}+3 \mathcal{H}(\rho+P)=0$ juntamente com as relações $\omega=P / \rho$ e $c_{s}^{2}=\delta P / \delta \rho$, temos que a equação (3.43) pode ser expressa como

$$
\delta^{\prime}+3 \mathcal{H}\left(c_{s}^{2}-\omega\right) \delta=-(1+\omega)\left(\theta+3 \Phi^{\prime}\right) .
$$

Essa equação é conhecida como a equação de continuidade perturbada. 
A equação $\delta T_{\nu ; \mu}^{\mu}=0$ para $\nu=i$ nos dá que

$$
\delta q^{\prime}+3 \mathcal{H} \delta q=-a \delta P-(\rho+P) a \Psi
$$

onde $\delta q \equiv a(\rho+P) v$ e $v$ é o potencial da velocidade relacionado a $v^{i}$ através de $v^{i}=\nabla^{i} v$. Escrevendo a equação (3.45) em termos de $v^{i}$ e tomando a divergência $\nabla_{i}$, obtemos

$$
\theta^{\prime}+\left[\mathcal{H}(1-3 \omega)+\frac{\omega^{\prime}}{1+\omega}\right] \theta=-\nabla^{2}\left(\frac{c_{s}^{2}}{1+\omega} \delta+\Psi\right)
$$

Para facilitar a manipulação das equações, trabalharemos no espaço de Fourier. Isso significa que todas as perturbações serão expandidas em função de suas transformadas de Fourier:

$$
\begin{gathered}
\Phi=\int \mathrm{d}^{3} k e^{i \mathbf{k} \cdot \mathbf{r}} \Phi_{k}, \quad \Psi=\int \mathrm{d}^{3} k e^{i \mathbf{k} \cdot \mathbf{r}} \Psi_{k} . \\
\delta=\int \mathrm{d}^{3} k e^{i \mathbf{k} \cdot \mathbf{r}} \delta_{k}, \quad \theta=\int \mathrm{d}^{3} k e^{i \mathbf{k} \cdot \mathbf{r}} \theta_{k} .
\end{gathered}
$$

O subscrito $k$ representa um modo de Fourier para cada número de onda $k$. Note que, em nossa notação, $k$ é uma quantidade comóvel que se mantém fixa. A seguir, descartaremos o subscrito $k$ por questão de simplicidade na notação. No espaço de Fourier, assumiremos que as variáveis de perturbação $(\delta, \theta, \Psi$, $\Phi$, etc.) são somas de ondas planas $A_{k} e^{i \mathbf{k} \cdot \mathbf{r}}$. Como as equações são lineares, cada onda plana obedece a equações idênticas, mas com números de onda comóveis $k$ diferentes. Durante a evolução linear, a escala física $\lambda_{p}$ da perturbação expande-se com a expansão cósmica com $\lambda_{p}=(2 \pi / k) a$. Obviamente, se as perturbações entrarem em um regime não-linear, então o tratamento descrito deixa de ser válido e as perturbações se desacoplam da expansão do universo e começam a se colapsar nas estruturas que compõe o universo, como estrelas e galáxias.

Cada quantidade perturbada $\phi$ e suas derivadas devem, ao passarmos para o espaço de Fourier, ser substituídas nas equações escritas no espaço das posições pelas relações

$$
\begin{gathered}
\phi(x, \eta) \rightarrow e^{i \mathbf{k} \cdot \mathbf{r}} \phi(\eta), \\
\nabla \phi(x, \eta) \rightarrow i e^{i \mathbf{k} \cdot \mathbf{r}} \mathbf{k} \phi(\eta),
\end{gathered}
$$




$$
\nabla^{2} \phi(x, \eta) \equiv \nabla_{i} \nabla^{i} \phi(x, \eta) \rightarrow-e^{i \mathbf{k} \cdot \mathbf{r}} k^{2} \phi(\eta)
$$

Note que somamos sobre dois índices espaciais sem o uso dos coeficientes da métrica $g_{i j}$ (mais exatamente, usamos a métrica tridimensional espacial induzida pelas simetrias do espaço-tempo homogêneo e isotrópico, que, para espaços planos, é igual à métrica euclidiana).

Das equações (3.37)-(3.40), (3.44) e (3.46), obtemos as seguintes equações para cada modo de Fourier,

$$
\begin{aligned}
& k^{2} \Phi+3 \mathcal{H}\left(\Phi^{\prime}-\mathcal{H} \Psi\right)=4 \pi G a^{2} \rho \delta \\
& k^{2}\left(\Phi^{\prime}-\mathcal{H} \Psi\right)=-4 \pi G a^{2}(1+\omega) \rho \theta \\
& \Psi=-\Phi \\
& \Phi^{\prime \prime}+2 \mathcal{H} \Phi^{\prime}-\mathcal{H} \Psi^{\prime}-\left(\mathcal{H}^{2}+2 \mathcal{H}^{\prime}\right) \Psi=-4 \pi G a^{2} c_{s}^{2} \rho \delta \\
& \delta^{\prime}+3 \mathcal{H}\left(c_{s}^{2}-\omega\right) \delta=-(1+\omega)\left(\theta+3 \Phi^{\prime}\right) \\
& \theta^{\prime}+\left[\mathcal{H}(1-3 \omega)+\frac{\omega^{\prime}}{1+\omega}\right] \theta=k^{2}\left(\frac{c_{s}^{2}}{1+\omega} \delta+\Psi\right),
\end{aligned}
$$

onde agora temos que

$$
\theta=i \mathbf{k} \cdot \mathbf{v}
$$

Embora as seis equações acima não sejam independentes, todas elas são úteis para entendermos a evolução das perturbações lineares.

Finalmente, podemos combinar as equações (3.52) e (3.53) para obtermos a equação de Poisson relativística

$$
k^{2} \Phi=4 \pi G a^{2} \rho\left[\delta+3 \mathcal{H}(\omega+1) \theta / k^{2}\right]=4 \pi G a^{2} \rho \delta^{*},
$$

onde definimos a variável relacionada à matéria-total [18],

$$
\delta^{*} \equiv \delta+3 \mathcal{H}(\omega+1) \theta / k^{2}
$$

Combinando as equações (3.52), (3.54) e (3.55), obtemos uma equação para $\Phi$,

$$
\Phi^{\prime \prime}+3 \mathcal{H}\left(1+c_{s}^{2}\right) \Phi^{\prime}+\left(c_{s}^{2} k^{2}+3 \mathcal{H}^{2} c_{s}^{2}+2 \mathcal{H}^{\prime}+\mathcal{H}^{2}\right) \Phi=0
$$

Utilizando a equação de Poisson relativística (3.59), essa expressão torna-se uma equação para $\delta^{*}[18]$, 


$$
\left(\delta^{*}\right)^{\prime \prime}+\mathcal{H}\left(1+3 c_{s}^{2}-6 \omega\right)\left(\delta^{*}\right)^{\prime}-\left[\frac{3}{2} \mathcal{H}^{2}\left(1-6 c_{s}^{2}-3 \omega^{2}+8 \omega\right)-c_{s}^{2} k^{2}\right] \delta^{*}=0
$$

onde $c_{s}^{2}$ e $\omega$ são funções arbitrárias do tempo e onde utilizamos a relação

$$
\mathcal{H}^{\prime}=-\frac{1}{2}(1+3 \omega) \mathcal{H}^{2}
$$

\subsubsection{Escalas Maiores do que o Horizonte}

Agora que derivamos as equações para as perturbações, tentaremos resolvê-las analiticamente para entendermos fisicamente o que elas representam. Entretanto, para conseguirmos isso, teremos que fazer algumas aproximações. Primeiramente, trabalharemos no limite de larga escala, isto é, $k \ll \mathcal{H}=a H$. Isso corresponde à escala na qual o comprimento de onda físico $\lambda_{p}=(2 \pi / k) a$ das perturbações é muito maior do que o raio de Hubble $H^{-1}$, isto é, as escalas físicas estão fora do horizonte. Se a pressão depender somente da densidade de energia e a equação de estado $\omega$ for uma constante, então temos que $c_{s}^{2}=\omega$, o que é válido para o caso da matéria e da radiação. Nesse caso, a equação (3.61), usando a equação (3.63), reduz-se a

$$
\Phi^{\prime \prime}+3 \mathcal{H}\left(1+c_{s}^{2}\right) \Phi^{\prime}=0 .
$$

Assim, vemos que $\Phi^{\prime}=0$ é uma solução. A equação (3.52) torna-se

$$
3 \mathcal{H}^{2} \Phi=4 \pi G a^{2} \rho \delta .
$$

Usando a equação de Friedmann, $3 \mathcal{H}^{2}=8 \pi G \rho a^{2}$, temos que

$$
\delta=2 \Phi
$$

Dessa maneira, $\Phi=$ constante, no limite de larga escala, implica em $\delta=$ constante. A equação (3.64) é uma equação diferencial de segunda ordem, portanto, deve ter duas soluções. Contudo, como a segunda solução decai no tempo, temos que $\Phi=$ constante é a solução dominante (pelo menos para $c_{s}^{2}>-1$ ). Assim, vemos que o potencial gravitacional mantém-se constante em escalas fora do raio de Hubble sempre que temos que $c_{s}^{2}=\omega$ para o fluido total que descreve a matéria do universo. 


\subsubsection{Escalas Menores do que o Horizonte}

Agora, estudaremos o caso oposto do estudado na seção anterior, $k \gg \mathcal{H}$, isto é, escalas dentro do raio de Hubble. No caso em que não temos uma força que impeça a acreção causada pela gravidade, a flutuação na densidade de energia de um fluido sem pressão pode crescer indefinidamente. Em geral, porém, a pressão do fluido resiste à gravidade e o colapso em algum momento termina. É interessante, portanto, derivar as equações para um fluido que não tenha pressão $(\omega=0)$ na ausência de perturbações, mas que tenha uma pequena velocidade do som em nível perturbativo,

$$
c_{s}^{2}=\frac{\delta P}{\delta \rho} \ll 1 .
$$

Dessa maneira a equação (3.53) nos diz que $\Phi^{\prime}-\mathcal{H} \Psi \simeq 0$, de forma que a equação (3.52) corresponde à equação de Poisson

$$
k^{2} \Phi=4 \pi G a^{2} \rho \delta=\frac{3}{2} \mathcal{H}^{2} \delta .
$$

Tomando a derivada em relação ao tempo conforme da equação (3.68) e substituindo o resultado na equação (3.56), obtemos

$$
\delta^{\prime}=-\theta-\frac{9}{2} \frac{\mathcal{H}^{2}}{k^{2}} \delta\left(2 \frac{\mathcal{H}^{\prime}}{\mathcal{H}}+\frac{\delta^{\prime}}{\delta}\right) \simeq-\theta
$$

Assim, as equações perturbadas, no limite em que as perturbações estão dentro do horizonte, tornam-se

$$
\begin{aligned}
& \delta^{\prime}=-\theta \\
& \theta^{\prime}=-\mathcal{H} \theta+c_{s}^{2} k^{2} \delta-k^{2} \Phi .
\end{aligned}
$$

Essas equações, juntamente com a equação (3.68), formam um sistema fechado de equações diferenciais. Diferenciando a equação (3.70) com respeito a $\eta$ e usando a equação (3.71), temos que

$$
\delta^{\prime \prime}+\mathcal{H} \delta^{\prime}+\left(c_{s}^{2} k^{2}-\frac{3}{2} \mathcal{H}^{2}\right) \delta=0
$$

No limite em que estamos em um espaço-tempo de Minkowski, $\mathcal{H} \rightarrow 0$, e essa equação reduz-se na equação clássica para fluidos $\delta^{\prime \prime}+c_{s}^{2} k^{2} \delta=0$, onde $c_{s}$ é a velocidade do som. A equação (3.72) mostra que a perturbação não crescerá se 


$$
c_{s}^{2} k^{2}-\frac{3}{2} \mathcal{H}^{2}>0
$$

isto é, se o comprimento de onda físico $\lambda_{p}=(2 \pi / k) a$ for menor que o comprimento de Jeans [18],

$$
\lambda_{J}=c_{s} \sqrt{\frac{\pi}{G \rho}} .
$$

Para escalas menores que $\lambda_{J}$, as perturbações estão sujeitas a uma oscilação amortecida. Para partículas da matéria escura, a dispersão da velocidade é sempre desprezível, pelo menos no regime de validade do tratamento linear. Para os fótons, temos que $c_{s}=c / \sqrt{3}$, de modo que

$$
\lambda_{J} \approx H^{-1}
$$

Portanto, o crescimento das perturbações não ocorre para todas as escalas menores que o raio de Hubble. Para os bárions, na época anterior ao desacoplamento dos fótons, a velocidade do som é comparável com a dos fótons, portanto, temos que as perturbações dos bárions são amortecidas e elas não crescem.

Quando $c_{s} k \ll \mathcal{H}$, as perturbações crescem livremente porque a gravidade é dominante quando comparada à pressão do fluido. A equação para um único fluido sem pressão e no limite dentro do horizonte torna-se

$$
\delta^{\prime \prime}+\mathcal{H} \delta^{\prime}-\frac{3}{2} \mathcal{H}^{2} \delta=0
$$

Na análise desse tipo de equações é comum empregar o número de $e$-foldings definido como $N=\ln a$ (lembrando que $a_{0}=1$ ) [18]. Dessa forma, a equação (3.76) pode ser escrita como

$$
\frac{\mathrm{d}^{2} \delta}{\mathrm{d} N^{2}}+\left(\frac{1}{\mathcal{H}} \frac{\mathrm{d} \mathcal{H}}{\mathrm{d} N}+1\right) \frac{\mathrm{d} \delta}{\mathrm{d} N}-\frac{3}{2} \delta=0 .
$$

A equação (3.63), por sua vez, pode ser escrita como

$$
\frac{1}{\mathcal{H}} \frac{\mathrm{d} \mathcal{H}}{\mathrm{d} N}=-\frac{1}{2}-\frac{3}{2} \omega
$$

Para um fluido sem pressão $(\omega=0)$, a equação (3.77) reduz-se, portanto, a

$$
\frac{\mathrm{d}^{2} \delta}{\mathrm{d} N^{2}}+\frac{1}{2} \frac{\mathrm{d} \delta}{\mathrm{d} N}-\frac{3}{2} \delta=0 .
$$

A solução analítica dessa equação pode ser obtida através da substituição direta de $\delta=A e^{\lambda N}$, o que nos dá a solução $\lambda=1,-3 / 2$. Então, a evolução do modo crescente 
e decrescente durante a era da matéria é dada por

$$
\delta_{+}=A a, \quad \delta_{-}=B a^{-3 / 2} .
$$

Em termos do tempo cósmico, o modo crescente evolui, na época dominada pela matéria, como $\delta_{+} \propto t^{2 / 3}$. O pré-fator é fixado pelas condições iniciais, que são estabelecidas durante o período inflacionário do universo. O modo decrescente logo se torna desprezível com respeito ao modo crescente e pode ser desconsiderado.

Inserindo $\delta_{+}$na equação de Poisson (3.68), vemos que $\Phi \propto a^{2} H^{2} \delta_{+} \propto a^{2} a^{-3} a^{1}$ $\propto$ constante. Portanto, o potencial gravitacional é constante durante a era dominada pela matéria, dentro ou fora do horizonte.

\subsection{Solução Cosmológica com Dois Fluidos}

Nesta seção, generalizaremos o caso de um único fluido para o caso mais realista em que tanto matéria $\left(\omega_{m}=c_{s}^{2}=0\right)$ quanto a radiação $\left(\omega_{r}=c_{s}^{2}=1 / 3\right)$ - sendo que consideraremos como radiação todo componente que tenha massa igual a zero ou que seja relativístico - estão presentes. Como estamos considerando a matéria escura como sendo o componente dominante da matéria, por ora, não consideraremos um termo explícito de interação entre a matéria e a radiação. A fração bariônica da matéria depois do redshift $z \approx 1000$ está efetivamente desacoplada da radiação, entretanto, antes dessa época ela pode ser considerada como parte de um plasma relativístico fóton-bárion. No espaço de Fourier, temos que o sistema de equações acopladas para as perturbações é dado por

$$
\begin{aligned}
& \delta_{m}^{\prime}=-\left(\theta_{m}+3 \Phi^{\prime}\right) \\
& \theta_{m}^{\prime}=-\mathcal{H} \theta_{m}-k^{2} \Phi \\
& \delta_{r}^{\prime}=-\frac{4}{3}\left(\theta_{r}+3 \Phi^{\prime}\right) \\
& \theta_{r}^{\prime}=k^{2}\left(\frac{3}{4} c_{s}^{2} \delta_{r}-\Phi\right) \\
& k^{2}\left(\Phi^{\prime}+\mathcal{H} \Phi\right)=-4 \pi G\left(1+\omega_{\mathrm{ef}}\right) a^{2} \rho_{t} \theta_{t} \\
& k^{2} \Phi+3 \mathcal{H}\left(\Phi^{\prime}+\mathcal{H} \Phi\right)=4 \pi G a^{2} \rho_{t} \delta_{t},
\end{aligned}
$$

onde definimos [18] 


$$
\begin{aligned}
& \rho_{t}=\rho_{m}+\rho_{r} \\
& \omega_{\mathrm{ef}}=\Omega_{r} \omega_{r}+\Omega_{m} \omega_{m}=\frac{\rho_{r} / 3}{\rho_{m}+\rho_{r}} \\
& \theta_{t}=\frac{\left(1+\omega_{m}\right) \Omega_{m} \theta_{m}+\left(1+\omega_{r}\right) \Omega_{r} \theta_{r}}{1+\omega_{\mathrm{ef}}} \\
& \delta_{t}=\Omega_{m} \delta_{m}+\Omega_{r} \delta_{r} .
\end{aligned}
$$

A equação de estado efetiva $\omega_{\mathrm{ef}}=P_{t} / \rho_{t}$ é dada por

$$
\omega_{\mathrm{ef}}=-1-\frac{2}{3} \frac{\dot{H}}{H^{2}}
$$

Na escala em que as perturbações são muito menores do que o horizonte, $k \gg \mathcal{H}$, a equação (3.86) nos dá

$$
k^{2} \Phi \simeq 4 \pi G a^{2}\left(\rho_{m} \delta_{m}+\rho_{r} \delta_{r}\right)=\frac{3}{2} \mathcal{H}^{2}\left(\Omega_{m} \delta_{m}+\Omega_{r} \delta_{r}\right)
$$

Seguindo os mesmos passos que nos levaram à equação (3.72), temos as seguintes equações para as perturbações bem menores do que o horizonte

$$
\begin{aligned}
& \delta_{m}^{\prime \prime}+\mathcal{H} \delta_{m}^{\prime}-\frac{3}{2} \mathcal{H}^{2}\left(\Omega_{m} \delta_{m}+\Omega_{r} \delta_{r}\right)=0 \\
& \delta_{r}^{\prime \prime}+\frac{k^{2}}{3} \delta_{r}=0
\end{aligned}
$$

Durante a época dominada pela radiação, temos que $\Omega_{m} \simeq 0$ e $\Omega_{r} \simeq 1$. A segunda equação mostra que o contraste de densidade da radiação oscila rapidamente ao redor de zero (já que estamos no limite em que $k^{2} \gg \mathcal{H}$ ). O mesmo é válido para o plasma acoplado de bárions e fótons. Portanto, podemos tomar a média das oscilações da perturbação da radiação e considerarmos $\left\langle\delta_{r}\right\rangle \simeq 0$ na primeira equação. Temos assim que $\Omega_{m} \delta_{m}+\Omega_{r} \delta_{r} \simeq 0$ e que

$$
\delta_{m}^{\prime \prime}+\mathcal{H} \delta_{m}^{\prime} \simeq 0
$$

A solução para esta equação é dada por $\delta_{m}=C_{1}+C_{2} \int \mathrm{d} \eta / a$. Durante a era da radiação, a integral $\int \mathrm{d} \eta / a$ representa apenas uma correção logarítmica ao resultado que obtivemos com somente um fluido de matéria. Assim, a perturbação da matéria não-relativística cresce lentamente durante a era da radiação.

Se supusermos a matéria escura fria $\left(\delta_{c}\right)$ e a matéria bariônica $\left(\delta_{b}\right)$ ao invés da 
matéria e da radiação, a equação (3.93) pode ser generalizada como

$$
\begin{aligned}
\delta_{c}^{\prime \prime}+\mathcal{H} \delta_{c}^{\prime}-\frac{3}{2} \mathcal{H}^{2}\left(\Omega_{c} \delta_{c}+\Omega_{b} \delta_{b}\right) & =0 \\
\delta_{b}^{\prime \prime}+\mathcal{H} \delta_{b}^{\prime}-\frac{3}{2} \mathcal{H}^{2}\left(\Omega_{c} \delta_{c}+\Omega_{b} \delta_{b}\right) & =0 .
\end{aligned}
$$

Como os bárions correspondem a uma pequena fração da matéria total, podemos assumir que $\left|\Omega_{b} \delta_{b}\right| \ll\left|\Omega_{c} \delta_{c}\right|$. Isso mostra que a equação (3.96) desacopla-se de $\delta_{b}$ e reduz-se à equação padrão para perturbações da matéria. Contudo, a equação para os bárions depende de $\Omega_{c} \delta_{c}$. Para essa equação diferencial, que está acoplada à equação para a matéria escura, a solução assintótica de $\delta_{b}$ se aproximará da de $\delta_{c}$.

Podemos, analogamente, considerar o caso de matéria sem pressão com uma constante cosmológica $\Lambda$. Contudo, $\rho_{\Lambda}$ é constante por definição e $\delta_{\Lambda}=0$, de modo que a equação (3.76) torna-se

$$
\delta_{m}^{\prime \prime}+\mathcal{H} \delta_{m}^{\prime}-\frac{3}{2} \mathcal{H}^{2} \Omega_{m} \delta_{m}=0 .
$$

Essa equação pode ser escrita em termos da derivada em relação a $N$,

$$
\frac{\mathrm{d}^{2} \delta_{m}}{\mathrm{~d} N^{2}}+\left(\frac{1}{\mathcal{H}} \frac{\mathrm{d} \mathcal{H}}{\mathrm{d} N}+1\right) \frac{\mathrm{d} \delta_{m}}{\mathrm{~d} N}-\frac{3}{2} \Omega_{m} \delta_{m}=0 .
$$

Se assumirmos que $\Omega_{m}=$ constante, então temos que a solução é dada por $\delta_{m} \sim a^{\lambda_{ \pm}}$ com [18]

$$
\lambda_{ \pm}=\frac{1}{4}\left(-1 \pm \sqrt{1+24 \Omega_{m}}\right) .
$$

Esse caso ocorre quando a fração $1-\Omega_{m}$ da matéria do Universo está na forma de uma densidade de energia que tem $\omega \approx 0$, mas que, ao contrário da matéria escura fria, não se aglomera em escalas menores que o horizonte. Um exemplo desse tipo de matéria são os neutrinos massivos depois que eles se tornam não-relativísticos. Para o caso em que a equação de estado da energia escura é $\omega=-1$, pode-se encontrar uma solução aproximada para a perturbação de densidade da matéria que é dada pela taxa de crescimento $f$, que é definida como [20]

$$
f \equiv \frac{\mathrm{d} \ln \delta_{m}}{\mathrm{~d} \ln a}=\Omega_{m}^{\gamma}
$$

Essa solução aproximada é

$$
\delta_{m}(a)=\delta_{m}\left(a_{i}\right) \exp \left(\int_{a_{i}}^{a} \Omega_{m}(\tilde{a})^{\gamma} \frac{\mathrm{d} \tilde{a}}{\tilde{a}}\right)
$$


Para o modelo $\Lambda$ CDM, o índice de crescimento é dado por $\gamma \approx 0,55$ [20]. Com esse comportamento, vemos que o termo $\mathcal{H}^{2} \delta_{m}$ na equação de Poisson, (3.92), não é mais constante, portanto, o potencial gravitacional $\Phi$ em escalas menores que a do horizonte também não é mais constante. Para o modelo $\Lambda$ CDM, o potencial é aproximadamente constante durante a era da matéria, mas começa a decrescer depois que o universo entra na era dominada pela energia escura.

\subsection{Equação de Boltzmann para Bárions, Fótons e Neutrinos}

Até o momento, consideramos somente fluidos perfeitos com estresse anisotrópico igual a zero, isto é, $T_{j}^{i}=0$, e que não interagem entre si a não ser via gravidade. Por causa disso, a dinâmica das perturbações desses fluidos foi descrita completamente por funções dependentes do módulo do número de onda $k$, do contraste da densidade e do gradiente do potencial de velocidade.

O universo, contudo, contém fluidos imperfeitos para os quais esse tipo de descrição é insuficiente. Quando os termos de interação ou o tensor de energia-momento dependem do vetor de momento total $\mathbf{P}$, o fluido precisa ser descrito por sua função de distribuição total $f(\mathbf{P}, \mathbf{x}, t)$. Radiação, bárions e neutrinos são fluidos imperfeitos e precisam de um tratamento mais sofisticado nesse sentido. Esse tratamento é extremamente útil para descrever o universo jovem. Consequentemente, ele é fundamental para descrever as anisotropias da radiação cósmica de fundo. Mas, para o universo tardio, o formalismo mais simples dos fluidos perfeitos continua válido e é uma ótima aproximação para a realidade.

Nesta seção, adotaremos a métrica perturbada com o tempo cósmico $t=\int a \mathrm{~d} \eta$, isto é,

$$
\mathrm{d} s^{2}=-(1+2 \Psi) \mathrm{d} t^{2}+a^{2}(t)(1+2 \Phi) \delta_{i j} \mathrm{~d} x^{i} \mathrm{~d} x^{j} .
$$

Dada uma função de distribuição $f(\mathbf{P}, \mathbf{x}, t)$, o tensor de energia-momento para um fluido é [10]

$$
T_{\nu}^{\mu}(\mathbf{x}, t)=\frac{g_{i}}{(2 \pi)^{3}} \int \mathrm{d} P_{1} \mathrm{~d} P_{2} \mathrm{~d} P_{3} \sqrt{-g} \frac{P^{\mu} P_{\nu}}{P^{0}} f(\mathbf{P}, \mathbf{x}, t),
$$

onde $P^{0} \equiv \mathrm{d} t / \mathrm{d} \lambda, P^{i} \equiv \mathrm{d} x^{i} / \mathrm{d} \lambda$ ( $\lambda$ é um parâmetro que caracteriza a trajetória da partícula) e $g_{i}$ são os graus de liberdade internos da partícula. Para uma partícula de massa $m$, temos que $P^{\mu} P_{\mu}=-m^{2}$. Através da expressão (3.104), podemos determinar a densidade de energia $-T_{0}^{0}$ e a pressão $T_{i}^{i} / 3$. Vemos que o termo que 
contribui para o estresse anisotrópico do fluido é proporcional a

$$
\frac{P^{i} P_{j}}{\left(P^{0}\right)^{2}}=\frac{\mathrm{d} x^{i} \mathrm{~d} x_{j}}{\mathrm{~d} t^{2}}=v^{i} v_{j}
$$

Essa é uma quantidade de segunda ordem, portanto, é desprezível para partículas massivas no formalismo das perturbações lineares. Para partículas de massa zero, contudo, isso não é verdade. Fótons e neutrinos (contanto que relativísticos) contribuem para o termo de anisotropia do tensor de energia-momento. Por causa do vínculo em relação à massa de $P^{\mu}$, existem somente três graus de liberdade para o momento. Podemos escolher esses graus de liberdade como sendo a magnitude espacial [8]

$$
p^{2} \equiv g_{i j} P^{i} P^{j}
$$

e o vetor de direção unitário

$$
\hat{p}^{i} \equiv P^{i} /|P|,
$$

de modo que $\delta_{i j} \hat{p}^{i} \hat{p}^{j}=1$.

Colocando $P^{i}=|P| \hat{p}^{i}$ na equação (3.106), achamos que

$$
p^{2}=a^{2}(1+2 \Phi)\left(\delta_{i j} \hat{p}^{i} \hat{p}^{j}\right) P^{2}=a^{2}(1+2 \Phi) P^{2} .
$$

Em primeira ordem, essa expressão nos dá que $|P|=p(1-\Phi) / a$. Assim, o vetor espacial $P^{i}$ pode ser escrito como

$$
P^{i}=\frac{1-\Phi}{a} p \hat{p}^{i}
$$

Os fótons satisfazem a relação $g_{\mu \nu} P^{\mu} P^{\nu}=0$, que se traduz na condição

$$
-(1+2 \Psi)\left(P^{0}\right)^{2}+p^{2}=0
$$

Obtemos, assim, a componente temporal de $P^{\mu}$,

$$
P^{0}=p(1-\Psi)
$$

O processo de colisões entre partículas pode ser descrito através da equação de Boltzmann [8] 


$$
\begin{aligned}
\frac{\mathrm{d} f}{\mathrm{~d} t} & =\frac{\partial f}{\partial t}+\frac{\partial f}{\partial x^{i}} \frac{\mathrm{d} x^{i}}{\mathrm{~d} t}+\frac{\partial f}{\partial p} \frac{\mathrm{d} p}{\mathrm{~d} t}+\frac{\partial f}{\partial \hat{p}^{i}} \frac{\mathrm{d} \hat{p}^{i}}{\mathrm{~d} t} \\
& =C[f],
\end{aligned}
$$

onde $f\left(p, \hat{p}^{i}, x^{i}, t\right)$ é a função de distribuição e $C[f]$ descreve o termo de colisão entre as partículas. O último termo da primeira linha da equação (3.112) anulase em primeira ordem, já que tanto $\partial f / \partial \hat{p}^{i}$ quanto $\mathrm{d} \hat{p}^{i} / \mathrm{d} t$ são termos de primeira ordem nas perturbações. O termo de colisão depende da partícula na qual estamos interessados, para os fótons, por exemplo, ele é dado pela interação Compton entre fótons e bárions.

Agora, consideraremos a equação de Boltzmann (3.112) para os fótons. Das equações (3.109) e (3.111), temos que

$$
\frac{\mathrm{d} x^{i}}{\mathrm{~d} t}=\frac{P^{i}}{P^{0}}=\frac{1-\Phi-\Psi}{a} \hat{p}^{i} .
$$

Através da componente temporal da equação geodésica $\mathrm{d} P^{0} / \mathrm{d} \lambda=-\Gamma_{\mu \nu}^{0} P^{\mu} P^{\nu}$, obtemos a expressão

$$
\frac{\mathrm{d} p}{\mathrm{~d} t}=-p\left(H+\frac{\partial \Phi}{\partial t}+\frac{\hat{p}^{i}}{a} \frac{\partial \Psi}{\partial x^{i}}\right)
$$

onde a relação $\mathrm{d} t / \mathrm{d} \lambda=P^{0}$ foi utilizada. Temos, então, que o lado esquerdo da equação de Boltzmann (3.112) torna-se, mantendo somente termos lineares,

$$
\frac{\mathrm{d} f}{\mathrm{~d} t}=\frac{\partial f}{\partial t}+\frac{\hat{p}^{i}}{a} \frac{\partial f}{\partial x^{i}}-p \frac{\partial f}{\partial p}\left(H+\frac{\partial \Phi}{\partial t}+\frac{\hat{p}^{i}}{a} \frac{\partial \Psi}{\partial x^{i}}\right)
$$

No universo de fundo não-perturbado, os fótons com temperatura $T$ possuem a função de distribuição de Bose-Einstein [8]

$$
f^{(0)}(t, p)=\frac{1}{[\exp (p / T)-1]},
$$

onde o potencial químico $\mu$ foi desprezado. No fundo cosmológico, a temperatura $T$ depende somente do tempo $t: T \propto 1 / a(t)$. No universo perturbado, por sua vez, podemos definir a perturbação da temperatura como

$$
\Theta\left(t, \mathbf{x}, \hat{p}^{i}\right) \equiv \delta T / T
$$

A partir de agora, essa será a perturbação para o fóton que ficará no lugar de $\delta_{\gamma}=4 \delta T / T$. Assumiremos que $\Theta$ não depende da magnitude $p$ do momento linear 
já que para o espalhamento Compton ela é aproximadamente conservada. Assim, a função de distribuição é dada por

$$
f(t, p, \mathbf{x}, \hat{p})=\left[\exp \left[\frac{p}{T(t)\left[1+\Theta\left(t, \mathbf{x}, \hat{p}^{i}\right)\right]}\right]-1\right]^{-1} .
$$

Se $\Theta \ll 1$, podemos expandir essa expressão ao redor de $f^{(0)}$ ao usarmos a relação $T \partial f^{(0)} / \partial T=-p \partial f^{(0)} / \partial p[8]$

$$
f=f^{(0)}-p \frac{\partial f^{(0)}}{\partial p} \Theta .
$$

Colocando a equação (3.119) na equação (3.115) e tomando somente termos de primeira ordem, temos que

$$
\frac{\mathrm{d} f^{(1)}}{\mathrm{d} t}=-p \frac{\partial f^{(0)}}{\partial p}\left[\frac{\partial \Theta}{\partial t}+\frac{\hat{p}^{i}}{a} \frac{\partial \Theta}{\partial x^{i}}+\frac{\partial \Phi}{\partial t}+\frac{\hat{p}^{i}}{a} \frac{\partial \Psi}{\partial x^{i}}\right]
$$

Podemos expandir $\Theta$ no espaço de Fourier,

$$
\Theta(\mathbf{r})=\frac{1}{(2 \pi)^{3}} \int \mathrm{d}^{3} k \Theta_{k} e^{i \mathbf{k} \cdot \mathbf{r}} .
$$

Com a dependência no tempo do lado esquerdo dessa equação implícita. Ao invés do vetor unitário $\hat{p}$, podemos utilizar o cosseno diretor [8]

$$
\mu=\frac{\mathbf{k} \cdot \hat{p}}{k}
$$

onde $k=|\mathbf{k}|$.

É conveniente integrar a dependência angular de $\Theta(k)$ e definir os multipolos da perturbação na temperatura [8],

$$
\Theta_{\ell} \equiv \frac{1}{(-i)^{\ell}} \int_{-1}^{1} \frac{\mathrm{d} \mu}{2} \mathcal{P}_{\ell}(\mu) \Theta(\mu),
$$

onde $\mathcal{P}_{\ell}$ é o polinômio de Legendre de ordem $\ell$.

Agora, devemos determinar o termo de colisão $C[f]$ para os fótons, que é, como foi dito anteriormente, predominantemente dado pelo espalhamento Compton: $e^{-}(\vec{q})+$ $\gamma(\vec{p}) \leftrightarrow e^{-}\left(\vec{q}^{\prime}\right)+\gamma\left(\vec{p}^{\prime}\right)$. O termo de colisão, nesse caso, é dado por [8]

$$
\begin{aligned}
C[f(\vec{p})] & =\frac{1}{p} \int \frac{\mathrm{d}^{3} q}{(2 \pi)^{3} 2 E_{e}(q)} \int \frac{\mathrm{d}^{3} q^{\prime}}{(2 \pi)^{3} 2 E_{e}\left(q^{\prime}\right)} \int \frac{\mathrm{d}^{3} p^{\prime}}{(2 \pi)^{3} 2 E\left(p^{\prime}\right)}|\mathcal{M}|^{2}(2 \pi)^{4} \\
& \times \delta^{3}\left[\vec{p}+\vec{q}-\vec{p}^{\prime}-\vec{q}^{\prime}\right] \delta\left[E(p)+E_{e}(q)-E\left(p^{\prime}\right)-E_{e}\left(q^{\prime}\right)\right] \\
& \times\left[f_{e}\left(\vec{q}^{\prime}\right) f\left(\vec{p}^{\prime}\right)-f_{e}(\vec{q}) f(\vec{p})\right]
\end{aligned}
$$


Aqui, as funções delta garantem a conservação de energia e de momento, os termos $2 E$ no denominador são o resultado da integração na energia, ou podem ser vistos como necessários para essa expressão ser invariante de Lorentz, enquanto o fator $f_{e}\left(\vec{q}^{\prime}\right) f\left(\vec{p}^{\prime}\right)-f_{e}(\vec{q}) f(\vec{p})$ garante que elétrons e fótons são criados a partir de elétrons e fótons. A amplitude $\mathcal{M}$ é determinada pelo processo em questão, no nosso caso, o espalhamento Compton. Utilizando as ferramentas da eletrodinâmica quântica pode-se mostrar que $|\mathcal{M}|^{2}=8 \pi \sigma_{T} m_{e}^{2}$, onde $\sigma_{T}$ é a seção de choque de Thomson [8,21]. Da equação (3.124), obtemos

$$
C[f]=-p \frac{\partial f^{(0)}}{\partial p} n_{e} \sigma_{T}\left[\Theta_{0}-\Theta(\hat{p})+\hat{p} \cdot \mathbf{v}_{b}\right],
$$

onde $n_{e}$ é a densidade de elétrons e $\mathbf{v}_{b}$ é a velocidade dos elétrons. A dedução dessa expressão é longa e não muito iluminadora. Por essa razão, ela foi omitida aqui, mas pode ser encontrada na referência [8].

Igualando a equação (3.125) com a equação (3.120), temos que

$$
\frac{\partial \Theta}{\partial t}+\frac{\hat{p}^{i}}{a} \frac{\partial \Theta}{\partial x^{i}}+\frac{\partial \Phi}{\partial t}+\frac{\hat{p}^{i}}{a} \frac{\partial \Psi}{\partial x^{i}}=n_{e} \sigma_{T}\left[\Theta_{0}-\Theta(\hat{p})+\hat{p} \cdot \mathbf{v}_{b}\right] .
$$

No espaço de Fourier, temos a transformação $\partial \Theta / \partial x^{j} \rightarrow i k_{j} \Theta$. Ainda, assumindo o fluido como sendo irrotacional, a velocidade dos bárions $\mathbf{v}_{b}$ é dada na direção de $\mathbf{k}$, de forma que

$$
v_{b}^{i}=v_{b} k^{i} / k
$$

onde $v_{b}$ é o módulo da velocidade. Assim, a equação (3.126) pode ser escrita como

$$
\frac{\partial \Theta}{\partial t}+\frac{i k \mu}{a} \Theta+\frac{\partial \Phi}{\partial t}+\frac{i k \mu}{a} \Psi=n_{e} \sigma_{T}\left[\Theta_{0}-\Theta(\hat{p})+\mu v_{b}\right] .
$$

Em termos do tempo conforme $\eta$, essa equação reduz-se em

$$
\Theta^{\prime}+i k \mu \Theta+\Phi^{\prime}+i k \mu \Psi=-\tau_{\text {op }}^{\prime}\left[\Theta_{0}-\Theta(\hat{p})+\mu v_{b}\right]
$$

onde introduzimos a profundidade óptica que é definida como [8]

$$
\tau_{\mathrm{op}} \equiv \int_{\eta}^{\eta_{0}} n_{e} \sigma_{T} a \mathrm{~d} \eta
$$

O cálculo para o caso de partículas massivas pode ser feito utilizando o mesmo formalismo exposto acima. Desse modo, obtemos as equações de Boltzmann para os bárions. Porém, nesse caso, ao invés da magnitude do momento $p$, devemos utilizar a energia $E=\sqrt{p^{2}+m^{2}}$ como um dos graus de liberdade da partícula. Para a 
matéria não-relativística, todos os termos de ordem $v^{2}=(p / E)^{2}$ ou maior podem ser desprezados. Isso permite que escrevamos as equações de Boltzmann para a distribuição $f_{m}$ em termos do primeiro e do segundo momento, isto é, da densidade de número e da velocidade

$$
n_{m} \equiv \int \frac{\mathrm{d}^{3} p}{(2 \pi)^{3}} f_{m}, \quad v_{m}^{i} \equiv \frac{1}{n_{m}} \int \frac{\mathrm{d}^{3} p}{(2 \pi)^{3}} \frac{p \hat{p}^{i}}{E} f_{m} .
$$

Anteriormente, nós já derivamos o conjunto de equações para o fluido perfeito composto por uma matéria sem pressão e que é dado pelas equações (3.44) e (3.46). Para o caso dos bárions, essas equações são modificadas somente pelo termo de colisão da equação de Boltzmann. Esse termo contribui somente para a equação da velocidade (3.46), isto é, para o primeiro momento da distribuição dos bárions $f_{b}$, visto que o espalhamento Compton (que acopla os elétrons e os fótons) e o espalhamento Coulomb (que acopla os elétrons e os prótons) conservam o número de bárions. Portanto, a equação da velocidade ganha um termo adicional vindo do processo de espalhamento. No espaço de Fourier, o conjunto completo de equações acopladas para o plasma de fótons e bárions é dado por

$$
\begin{aligned}
\Theta^{\prime} & =-\Phi^{\prime}-i k \mu(\Theta+\Psi)-\tau_{\mathrm{op}}^{\prime}\left(\Theta_{0}-\Theta+\mu v_{b}\right) \\
\delta_{b}^{\prime} & =-i k v_{b}-3 \Phi^{\prime} \\
v_{b}^{\prime} & =-\mathcal{H} v_{b}-i k \Psi+\frac{\tau_{\mathrm{op}}}{R_{s}}\left(3 i \Theta_{1}+v_{b}\right)
\end{aligned}
$$

onde

$$
R_{s} \equiv \frac{3}{4} \frac{\rho_{b}}{\rho_{\gamma}}
$$

Note que utilizamos a relação $\theta_{b}=i k_{j} v_{b}^{j}=i k v_{b}$ para converter $\theta_{b}$ em $v_{b}$ nas equações (3.133) e (3.134). A dedução das equações dos bárions também foi omitida, pois ela repete passo a passo o cálculo que fizemos para os fótons com a a diferença que agora temos um termo de massa dos bárions e que além do termo de colisão do tipo Compton, temos também um termo de colisão do tipo Coulomb. Essa dedução, contudo, também pode ser encontrada na referência [8].

A matéria escura, em princípio, não sofre nenhuma interação eletromagnética, portanto, sua perturbação não é afetada por um termo de colisão, de forma que temos as seguintes equações para $\delta_{c}$ e $v_{c}$,

$$
\delta_{c}^{\prime}=-i k v_{c}-3 \Phi^{\prime}
$$




$$
v_{c}^{\prime}=-\mathcal{H} v_{c}-i k \Psi
$$

Agora, podemos derivar uma equação para o termo de monopolo da radiação $\Theta_{0}$. Multiplicando a equação (3.132), primeiramente, por $\mathcal{P}_{0}(\mu)$ e depois por $\mathcal{P}_{1}(\mu)$ e integrando as expressões resultantes em termos de $\mu$ no intervalo $[-1,1]$, obtemos, utilizando a definição (3.123) [8]

$$
\begin{gathered}
\Theta_{0}^{\prime}+k \Theta_{1}=-\Phi^{\prime}, \\
\Theta_{1}^{\prime}-\frac{k}{3}\left(\Theta_{0}+\Psi\right)=\tau_{\text {op }}^{\prime}\left(\Theta_{1}-\frac{i}{3} v_{b}\right),
\end{gathered}
$$

onde o quadrupolo $\Theta_{2}$ foi desprezado na segunda equação. No regime de acoplamento forte $\left(\tau_{\text {op }} \gg 1\right)$, temos que $\left|\tau_{\text {op }}^{\prime} \Theta_{1}\right| \gg\left|\Theta_{1}^{\prime}\right|$ na equação (3.139). Assim, o segundo termo, $(k / 3) \Theta_{0}$, na equação (3.139) é da mesma ordem que o lado direito da equação, dando-nos que $\Theta_{1} \sim k \Theta_{0} / \tau_{\text {op }}^{\prime} \sim\left(k \eta / \tau_{\text {op }}\right) \Theta_{0}$. No limite de acoplamento forte, isso significa que $\Theta_{1} \ll \Theta_{0}$ para os modos ao redor do raio de Hubble $(k \eta \sim 1)$. Essa propriedade geralmente é válida para qualquer momento de multipolo $\Theta_{\ell}$, isto é, $\Theta_{\ell+1} / \Theta_{\ell} \sim k \eta / \tau_{\text {op }}$. Portanto, é uma boa aproximação desprezar os momentos $\Theta_{\ell}$ $(\ell \geq 2)$ em relação ao monopolo e o dipolo no regime de acoplamento forte.

Ao reescrevermos a equação (3.134), ainda na aproximação de um acoplamento forte, na forma $v_{b}=-3 i \Theta_{1}+\left(R_{s} / \tau_{\text {op }}^{\prime}\right)\left[v_{b}^{\prime}+\mathcal{H} v_{b}+i k \Psi\right]$, temos que o termo $\left(R_{s} / \tau_{\text {op }}^{\prime}\right)\left[v_{b}^{\prime}+\right.$ $\left.\mathcal{H} v_{b}+i k \Psi\right]$ é suprimido relativo aos outros por um fator de $1 / \tau_{\text {op }}$, dando-nos a relação $v_{b} \simeq-3 i \Theta_{1}$ na menor ordem da teoria de perturbação. Substituindo essa relação na equação (3.134), temos que

$$
v_{b} \simeq-3 i \Theta_{1}-3 i \frac{R_{s}}{\tau_{\mathrm{op}}^{\prime}}\left(\Theta_{1}^{\prime}+\mathcal{H} \Theta_{1}-\frac{k}{3} \Psi\right) .
$$

Colocando a equação (3.140) na equação (3.139) e usando a derivada da equação (3.138) para eliminar o termo $\Theta_{1}^{\prime}$, temos que

$$
\Theta_{0}^{\prime \prime}+\frac{R_{s}}{1+R_{s}} \mathcal{H} \Theta_{0}^{\prime}+k^{2} c_{s}^{2} \Theta_{0}=-\frac{k^{2}}{3} \Psi-\frac{R_{s}}{1+R_{s}} \mathcal{H} \Phi^{\prime}-\Phi^{\prime \prime}
$$

onde

$$
c_{s}^{2} \equiv \frac{\delta P_{\gamma}}{\delta \rho_{\gamma}+\delta \rho_{b}}=\frac{1}{3\left(1+R_{s}\right)} .
$$

Essa é a velocidade do som efetiva do plasma acoplado de bárions e fótons.

Os potenciais gravitacionais $\Phi$ e $\Psi$ são determinados pela ação conjunta de todos 
os campos de matéria: bárions, matéria escura, fótons, neutrinos e energia escura. As perturbações da temperatura dos neutrinos, $\mathcal{N}\left(t, \mathbf{x}, \hat{p}^{i}\right) \equiv \delta T_{\nu} / T_{\nu}$, podem ser obtidas de forma análoga às obtidas para os fótons. A equação dinâmica para $\mathcal{N}$ é idêntica à equação (3.132) para a perturbação $\Theta$ dos fótons, só que, como os neutrinos não interagem eficientemente com os bárions, não temos o termo que depende da profundidade óptica. Se desprezarmos a contribuição da energia escura, aproximação válida no universo jovem, a componente (00) da equação de Einstein (3.52) é dada por

$$
k^{2} \Phi+3 \mathcal{H}\left(\Phi^{\prime}-\mathcal{H} \Psi\right)=4 \pi G a^{2}\left(\rho_{m} \delta_{m}+4 \rho_{r} \Theta_{r, 0}\right)
$$

onde

$$
\begin{gathered}
\rho_{m} \delta_{m} \equiv \rho_{b} \delta_{b}+\rho_{c} \delta_{c} \\
\rho_{r} \Theta_{r, i} \equiv \rho_{\gamma} \Theta_{i}+\rho_{\nu} \mathcal{N}_{i},
\end{gathered}
$$

onde $\Theta_{0}$ e $\mathcal{N}_{0}$ são os monopolos dos fótons e dos neutrinos de massa zero, respectivamente, que são relacionados ao tensor de energia momento através de $T_{(\gamma) 0}^{0}=$ $-\rho_{\gamma}\left(1+4 \Theta_{0}\right)$ e $T_{(\nu) 0}^{0}=-\rho_{\nu}\left(1+4 \mathcal{N}_{0}\right)$. Das equações $(3.53)$ e $(3.143)$, temos que

$$
k^{2} \Phi=4 \pi G a^{2}\left[\rho_{m} \delta_{m}+4 \rho_{r} \Theta_{r, 0}+\frac{3 \mathcal{H}}{k}\left(i \rho_{m} v_{m}+4 \rho_{r} \Theta_{r, 1}\right)\right]
$$

onde utilizamos o fato, como vimos anteriormente, que a velocidade $v_{r}$ para radiação é relacionada ao dipolo $\Theta_{r, 1}$ através de $v_{r}=-3 i \Theta_{r, 1}$.

Existe outra relação, oriunda do termo anisotrópico das equações de campo de Einstein, entre a soma dos potenciais gravitacionais com o estresse anisotrópico dos fótons e dos neutrinos que é dada por [8]

$$
k^{2}(\Phi+\Psi)=-32 \pi G a^{2} \rho_{r} \Theta_{r, 2} .
$$

Se o quadrupolo $\Theta_{r, 2}$ for desprezível, temos que $\Phi \simeq-\Psi$. A equação (3.147) assume uma forma particularmente simples durante a era dominada pela radiação e para escalas maiores que o horizonte. Durante a era dominada pela radiação e no regime de acoplamento forte, podemos utilizar a equação de Friedmann (2.15) no espaço plano para reescrever a equação (3.147) como

$$
k^{2}(\Phi+\Psi)=-12 \mathcal{H}^{2} f_{\nu} \mathcal{N}_{2}
$$


onde

$$
f_{\nu}=\frac{\rho_{\nu}}{\rho_{\nu}+\rho_{\gamma}}
$$

Na equação (3.148), utilizamos o fato que o termo dominante no quadrupolo $\Theta_{r, 2}$ é o relacionado com os neutrinos, já que o plasma de fótons e bárions comporta-se quase como um fluido perfeito, portanto, não possui um quadrupolo significante. Para condições adiabáticas iniciais, a flutuação na radiação em larga escala é igual à flutuação na parte dos neutrinos. Portanto, podemos colocar $\Theta_{r, 0}=\mathcal{N}_{0}$. Agora, da equação (3.143), assumindo $k \ll \mathcal{H}$ e $\Phi$ constante, e desprezando $\rho_{m}$, temos que

$$
3 \mathcal{H}^{2} \Psi=-16 \pi a^{2} \rho_{r} \Theta_{r, 0} .
$$

Temos, assim, que

$$
\Psi=-2 \Theta_{r, 0}=-2 \mathcal{N}_{0} .
$$

Isso substitui a equação (3.66) quando não podemos assumir $\Phi=-\Psi$. Para os neutrinos, podemos utilizar as equações (3.138) e (3.139) sem o acoplamento com os bárions. Contudo, o quadrupolo que desprezamos anteriormente na equação (3.139) agora tem que ser considerado. Multiplicando a equação (3.132) por $\mathcal{P}_{2}(\mu)$ e integrando com respeito a $\mu$, obtemos a equação para $\mathcal{N}_{2}$. Desprezando $\mathcal{N}_{3}$ para $k \ll \mathcal{H}$ e eliminando $\mathcal{N}_{1}$, temos que [18]

$$
\mathcal{N}_{2}^{\prime \prime}=\frac{2 k^{2}}{15}\left(\Psi+\mathcal{N}_{0}-2 \mathcal{N}_{2}\right)
$$

A equação (3.148) pode ser escrita como

$$
\mathcal{N}_{2}=A \mathcal{H}^{-2}
$$

onde $A=-k^{2}(\Phi+\Psi) /\left(12 f_{\nu}\right)$ é aproximadamente constante já que os potenciais são constantes em escalas maiores que o horizonte e $f_{\nu} \approx \Omega_{\nu} \approx$ constante durante a época dominada pela radiação. Diferenciando a equação (3.153) duas vezes com respeito a $\eta$ (lembrando que $\mathcal{H}^{\prime} / \mathcal{H}^{2} \simeq-1$ nesse regime), temos que

$$
\mathcal{N}_{2}^{\prime \prime}=2 A=-k^{2}(\Phi+\Psi) /\left(6 f_{\nu}\right) .
$$

Combinando as equações (3.151), (3.152) e (3.154) e tomando o limite $k \ll \mathcal{H}$, temos finalmente que 


$$
\Phi=-\Psi\left(1+\frac{2}{5} f_{\nu}\right)
$$

Essa é a condição inicial para a relação entre os potenciais em tempos remotos dominados pela radiação.

\subsection{O Espectro de Potência da Matéria}

As estruturas do universo, isto é, as galáxias e os aglomerados de galáxias, começaram a crescer substancialmente somente depois da época da igualdade entre a radiação e a matéria. Como a matéria não-relativística possui uma pressão desprezível em relação a sua densidade de energia, a atração gravitacional torna-se mais forte que a repulsão da pressão na época dominada pela matéria. As perturbações da matéria sem pressão, especialmente da matéria escura, são responsáveis pela formação das galáxias. Por sua vez, podemos quantificar a distribuição da matéria no Universo ao medirmos a função de correlação ou o espectro de potência das galáxias que observamos no céu.

Para podermos derivar o espectro das perturbações hoje, necessitamos saber a evolução de $\Phi(k, t)$ do universo jovem até a época presente. A inflação cósmica do universo extremamente jovem, que é necessária para resolver o problema da planura e do horizonte, é vista como a provável responsável por gerar as inomogeneidades primordiais do universo. Nos modelos mais simples para a inflação, essas inomogeneidades primordiais são formadas através das flutuações quânticas de um campo escalar canônico [22].

Durante a expansão acelerada do universo na época inflacionária, a escala $\lambda_{p}=$ $(2 \pi / k) a$ das perturbações torna-se maior do que o raio de Hubble $H^{-1}$, que é aproximadamente constante nesse período (estamos considerando somente as perturbações com comprimentos de onda menores que o raio de Hubble no início da inflação, que são as perturbações importantes para a formação de estruturas no universo observável). Fora do raio de Hubble (regime caracterizado por $k \lesssim \mathcal{H}=a H$ ), as perturbações tornam-se constantes. Assim, as perturbações, no regime seguinte à sua saída do raio de Hubble, evoluem somente de forma adiabática. Contudo, depois de findada a inflação, o raio comóvel de Hubble, $\mathcal{H}^{-1}$, começa a crescer assim que o universo começa uma expansão desacelerada $\left(a \propto t^{p}\right.$ com $\left.p<1\right)$. Durante as eras da radiação e da matéria, as perturbações cruzam novamente o raio de Hubble $(k \gtrsim \mathcal{H})$. A época do segundo cruzamento do raio de Hubble depende do comprimento de onda da perturbação. Esse cruzamento, obviamente, ocorre mais cedo para as escalas menores das perturbações. 
Durante a inflação, as perturbações da densidade são geradas de forma aproximadamente invariante quanto à escala pelas flutuações quânticas do campo escalar. São essas perturbações no campo escalar que geram as condições iniciais para o potencial gravitacional. O espectro de potência inicial de $\Phi$ gerado durante a inflação é dado por [22]

$$
P_{\phi}^{(i)} \equiv\left\langle\left|\Phi\left(k, a_{i}\right)\right|^{2}\right\rangle=\frac{50 \pi^{2}}{9 k^{3}}\left(\frac{k}{H_{0}}\right)^{n_{s}-1} \delta_{H}^{2},
$$

onde $n_{s}$ é o índice espectral e $\delta_{H}$ representa a amplitude do potencial. Esses parâmetros são dados pelo WMAP como sendo $n_{s}=0,9608 \pm 0,0080$ e $\delta_{H}^{2}=3,2 \times 10^{-10}$ [7]. O valor do índice espectral menor do que 1, isto é, um espectro não exatamente invariante em relação à escala da perturbação, é uma das previsões dos modelos de inflação com um campo escalar canônico. Vemos, assim, que a ideia de um universo inflacionário é robusta quando comparada com as anisotropias da radiação cósmica de fundo.

Para obtermos o potencial gravitacional hoje, precisamos resolver a equação para $\Phi(k, t)$ do início da época dominada pela radiação até o presente. A evolução do potencial gravitacional durante as eras cosmológicas remotas depende do modo $k$. $\mathrm{O}$ comprimento de onda $k_{\text {eq }}$ que caracteriza a fronteira entre modos de larga e pequena escala corresponde ao modo que entrou no raio de Hubble na época da igualdade entre a matéria e a radiação, isto é, $k_{\text {eq }}=a_{\text {eq }} H\left(a_{\text {eq }}\right)$. Utilizando (2.69) juntamente com $H\left(a_{\text {eq }}\right) / H_{0}=\left[2 \Omega_{m}^{(0)} / a_{\text {eq }}^{3}\right]^{1 / 2}$, temos que

$$
k_{\mathrm{eq}}=H_{0} \sqrt{\frac{2 \Omega_{m}^{(0)}}{a_{\mathrm{eq}}}}=0,073 \Omega_{m}^{(0)} h^{2} \mathrm{Mpc}^{-1} .
$$

Primeiramente, consideraremos os modos de larga escala com $k \ll k_{\text {eq }}$. Anteriormente, nós já vimos que existe uma solução com $\Phi=$ constante para um sistema de fluido único. Porém, ainda temos que verificar o que acontece durante a transição da era da radiação para a era da matéria. Aqui, desconsideraremos as contribuições dos bárions e do quadrupolo $\Theta_{r, 2}$. Na aproximação de escalas muito maiores que o horizonte $(k \ll \mathcal{H})$, temos as seguintes equações aproximadas vindas das equações (3.143), (3.136), e (3.138),

$$
\begin{aligned}
& 3 \mathcal{H}\left(\Phi^{\prime}+\mathcal{H} \Phi\right)=4 \pi G a^{2}\left(\rho_{c} \delta_{c}+4 \rho_{r} \Theta_{r, 0}\right) \\
& \delta_{c}^{\prime}=-3 \Phi^{\prime} \\
& \Theta_{r, 0}^{\prime}=-\Phi^{\prime} .
\end{aligned}
$$


Nas equações (3.158) e (3.160), $\Theta_{r, 0}$ inclui as contribuições tanto da radiação quanto dos neutrinos. Das equações (3.159) e (3.160), temos que $\delta_{c}=3 \Theta_{r, 0}+C$, onde $C$ é uma constante de integração. Como mencionamos anteriormente, as perturbações da radiação $\delta_{r}=\delta \rho_{r} / \rho_{r}$ são relacionadas às perturbações da matéria $\delta_{m}=\delta \rho_{m} / \rho_{m}$ através de $\delta_{r}=(4 / 3) \delta_{m}$; essa relação é facilmente derivada ao notarmos que $\rho_{r} \propto T^{4}$ e $\rho_{m} \propto T^{3}$. Isso implica que temos $\delta_{m}=3 \Theta_{r, 0}$, que é a condição adiabática para os fluidos. Portanto, temos que $C=0$ para matéria escura, enquanto a condição adiabática inicial for respeitada.

Colocando essa relação para $\Theta_{r, 0}$ na equação (3.158) e introduzindo a variável $y \equiv a / a_{\text {eq }}=\rho_{c} / \rho_{r}$, temos que [8]

$$
y \frac{\mathrm{d} \Phi}{\mathrm{d} y}+\Phi=\frac{3 y+4}{6(y+1)} \delta_{c},
$$

onde utilizamos que $\mathrm{d} y / \mathrm{d} \eta=\mathcal{H} y$ e $3 H^{2}=8 \pi G \rho_{c}(1+1 / y)$. Tomando a derivada em relação a y da equação (3.161) e usando a equação (3.159), temos que

$$
\frac{\mathrm{d}^{2} \Phi}{\mathrm{d} y^{2}}+\frac{21 y^{2}+54 y+32}{2 y(y+1)(3 y+4)} \frac{\mathrm{d} \Phi}{\mathrm{d} y}+\frac{1}{y(y+1)(3 y+4)} \Phi=0 .
$$

Essa equação diferencial possui a solução analítica $[8,23]$

$$
\Phi(y)=c_{1} \frac{\sqrt{y+1}}{y^{3}}+c_{2} \frac{9 y^{3}+2 y^{2}-8 y-16}{y^{3}} .
$$

Podemos determinar as constantes $c_{1}$ e $c_{2}$ ao impormos as condições $\Phi_{i}=\Phi(0)$ e $(\mathrm{d} \Phi / \mathrm{d} y)_{i}=0$. Essas condições nos dão $c_{1}=16 c_{2}=(8 / 5) \Phi(0)$, de modo que a solução é

$$
\Phi(y)=\Phi(0) \frac{9 y^{3}+2 y^{2}-8 y-16+16 \sqrt{y+1}}{10 y^{3}} .
$$

No limite em que $y=a / a_{\mathrm{eq}} \rightarrow \infty$, o potencial gravitacional aproxima-se de $\Phi \rightarrow$ $(9 / 10) \Phi(0)$. Portanto, para perturbações muito maiores que o horizonte, o potencial diminui em $10 \%$ durante a transição da era da radiação para a era da matéria.

Agora, consideraremos a evolução de $\Phi$ durante a época dominada pela radiação. Como $c_{s}^{2} \simeq 1 / 3, \mathcal{H}^{\prime} \simeq-\mathcal{H}^{2}$ e $\mathcal{H} \simeq 1 / \eta$ nesse regime, a equação (3.61) torna-se

$$
\Phi^{\prime \prime}+\frac{4}{\eta} \Phi^{\prime}+\frac{k^{2}}{3} \Phi=0
$$

A solução dessa equação que satisfaz as condições $\Phi=\Phi_{I}$ e $\mathrm{d} \Phi / \mathrm{d} \eta=0$ em $\eta=0$ é [8] 


$$
\Phi(k, \eta)=3 \Phi_{I} \frac{\sin (k \eta / \sqrt{3})-(k \eta / \sqrt{3}) \cos (k \eta / \sqrt{3})}{(k \eta / \sqrt{3})^{3}} .
$$

Para modos fora do raio de Hubble $(k \ll \mathcal{H}$, isto é, $k \eta \ll 1)$ temos que $\Phi(k, \eta) \simeq$ $\Phi_{I}\left[1-(k \eta)^{2} / 10\right]$, o que significa que o potencial é aproximadamente constante. Os modos com $k \gg k_{\text {eq }}$ cruzaram o raio de Hubble $(k \eta>1)$ antes da igualdade entre matéria e radiação. Essas perturbações de pequena escala começaram a decair depois de cruzarem o raio de Hubble. Assintoticamente (isto é, $k \eta \gg 1$ ), o potencial gravitacional decresce como $1 /(k \eta)^{2}$ com oscilações (durante a era da radiação). Para valores grandes de $k$ esse decaimento começou antes, o que faz com que a amplitude do potencial gravitacional resultante seja suprimida para as perturbações de pequena escala. Depois de o universo entrar a época dominada pela matéria, a amplitude de $\Phi$ aproxima-se de um valor constante.

Desse modo, mostramos que a evolução do potencial gravitacional depende da escala da perturbação. Para descrever a evolução de cada número de onda $k$ durante a época de transferência (da era da radiação para a época com $a=a_{T}$, isto é, uma época bem tardia quando a era da radiação já acabou, mas antes da época da aceleração cósmica), introduziremos a função de transferência

$$
T(k) \equiv \frac{\Phi\left(k, a_{T}\right)}{\Phi_{L E}\left(k, a_{T}\right)},
$$

onde $\Phi_{L E}\left(k, a_{T}\right)$ é a solução de larga escala dada por

$$
\Phi_{L E}\left(k, a_{T}\right)=\frac{9}{10} \Phi\left(k, a_{i}\right) .
$$

Note que, para $a>a_{T}$, a evolução de $\Phi$ torna-se independente de $k$ ( $\Phi=$ constante durante a era da matéria).

Ao resolvermos numericamente as equações para cada modo $k$, podemos ver que a função de transferência para grandes escalas é caracterizada por $T(x)=1$, onde $x=k / k_{\text {eq }}$, e para pequenas escalas por $T(k) \propto(\ln k) / k^{2}$, o que mostra que o potencial gravitacional $\Phi\left(k, a_{T}\right)$ é suprimido para grandes $k$ [8].

Quando $a>a_{T}$, o potencial gravitacional $\Phi$ mantém-se constante durante a era da matéria, porém depois do universo ter entrado na época da aceleração cósmica, $\Phi$ começa a variar. Para quantificar isso, introduziremos a função de crescimento $D(a)$,

$$
\frac{\Phi(a)}{\Phi\left(a_{T}\right)}=\frac{D(a)}{a} \quad\left(a>a_{T}\right) .
$$

No modelo $\Lambda$ CDM, a evolução constante de $\Phi(a)$ é seguida por um decaimento ao 
redor da era da matéria. Esse decaimento é associado com a diminuição tanto de $\Omega_{m}$ quanto da taxa de crescimento de $\delta_{m}$. Essa variação de $\Phi$ leva ao chamado efeito de Sachs-Wolfe integrado nas anisotropias da temperatura da radiação cósmica de fundo [8]. Esse efeito pode ser utilizado para discernir entre os diferentes modelos de energia escura.

Combinando as equações (3.167), (3.168), e (3.169), podemos determinar o potencial gravitacional hoje através de

$$
\Phi\left(k, a_{0}\right)=\frac{9}{10} \Phi\left(k, a_{i}\right) T(k) D\left(a_{0}\right),
$$

onde utilizamos $a_{0}=1$.

Depois de o universo entrar na era da matéria, a perturbação da radiação $\Theta_{r, 0}$ é desprezível em relação à perturbação da matéria $\delta_{m}$. Para o modelo $\Lambda$ CDM ou em qualquer modelo em que a energia escura não se aglomera, podemos também ignorar a perturbação da energia escura comparada com a da matéria. Na aproximação de escalas bem menores que o horizonte $(k \gg \mathcal{H})$, a equação $(3.143)$ reduz-se a

$$
k^{2} \Phi=4 \pi G a^{2} \rho_{m} \delta_{m}
$$

Usando as relações $\rho_{m}=\rho_{m}^{(0)} / a^{3}$ e $\Omega_{m}^{(0)}=8 \pi G \rho_{m}^{(0)} /\left(3 H_{0}^{2}\right)$, essa equação pode ser expressa como

$$
\delta_{m}(k, a)=\frac{2 k^{2} a}{3 \Omega_{m}^{(0)} H_{0}^{2}} \Phi(k, a) .
$$

Usando as equações (3.156), (3.170), e (3.172), o espectro de potência da matéria na presente época é

$$
P_{\delta_{m}} \equiv\left\langle\left|\delta_{m}(k, a)\right|^{2}\right\rangle=\frac{2 \pi^{2} \delta_{H}^{2}}{\left(\Omega_{m}^{(0)}\right)^{2}}\left(\frac{k}{H_{0}}\right)^{n_{s}} T^{2}(k) D^{2}\left(a_{0}\right) H_{0}^{-3} .
$$

Em grandes escalas $\left(k / k_{\text {eq }} \ll 1\right)$ o espectro de potência da matéria é dependente da escala $P_{\delta_{m}} \propto k^{n_{s}}$, e vemos que ele cresce com $k$. Em pequenas escalas $\left(k / k_{\text {eq }} \gg 1\right)$, temos que $P_{\delta_{m}} \propto k^{n_{s}-4}(\ln k)^{2}$, o que decresce com $k$. Portanto, o espectro de potência possui um pico cujo número de onda é determinado por $k_{\text {eq }}$.

O estudo do espectro de potência da matéria é um ótimo caminho para vincular os modelos de energia escura, visto que a dinâmica tardia do potencial gravitacional, $\Phi$, depende fortemente da evolução da energia escura. 


\section{Capítulo 4}

\section{Energia Escura}

Desde a publicação dos dados observacionais das supernovas do tipo Ia e de sua posterior análise [24,25], as evidências de que o universo está se expandindo aceleradamente somente se acumulam. A origem física para a presente aceleração cósmica do universo é o que denominamos energia escura. Apesar de anos de pesquisa, a origem da energia escura ainda não foi determinada de forma conclusiva [16]. Essa energia escura é facilmente distinguível da matéria ordinária, como bárions e radiação, visto que ela possui pressão negativa. Essa pressão negativa permite que a força da gravidade seja superada, o que faz o universo expandir-se aceleradamente. As observações das supernovas do tipo Ia mostraram que $70 \%$ da energia do presente universo consiste em energia escura. Neste capítulo, estudaremos, primeiramente, as observações que nos levam naturalmente a constatação da existência da energia escura no universo e depois detalharemos dois possíveis candidatos à energia escura: a constante cosmológica e um campo escalar canônico chamado de quintessência.

\subsection{Evidências Observacionais da Energia Escura}

A existência da energia escura é confirmada por várias observações. Entre elas temos as observações de supernovas, a radiação cósmica de fundo em microondas, as oscilações acústicas dos bárions (BAO), a estrutura em larga-escala do universo e também o fato que a idade do universo no modelo CDM é inferior à idade das estrelas mais velhas já encontradas. Mesmo antes da análise feita em 1998 das supernovas do tipo Ia, sabia-se que um universo dominado pela matéria escura possuía uma idade inferior à idade das estrelas mais antigas. A energia escura pode eliminar essa discrepância ao fazer o universo ser muito mais antigo que em um modelo com somente matéria escura e bariônica. Mas, definitivamente, a primeira grande evidência para a aceleração do universo atual foi obtida pela medição da distância 
de luminosidade de supernovas do tipo Ia. As observações da radiação cósmica de fundo (CMB) também são consistentes com a presença da energia escura, embora as observações da CMB sozinhas não possam indicar precisamente o domínio da energia escura no universo recente. As medidas de BAO também propiciaram outro teste independente da existência de energia escura. O espectro de potência da distribuição da matéria é outro observável que indica a existência da energia escura. A seguir, discutiremos brevemente algumas das evidências observacionais da energia escura.

\subsubsection{A Idade do Universo}

O inverso da constante de Hubble, $H_{0}$, é uma medida aproximada da idade do universo atual, $t_{0}$. Contudo, podemos calcular essa idade $t_{0}$ mais precisamente e compará-la com a idade dos objetos mais antigos do universo observável que temos conhecimento. Por simplicidade, consideraremos a equação de estado da energia escura igual a uma constante, de modo que $\rho_{d}=\rho_{d}^{(0)}(1+z)^{3\left(1+\omega_{d}\right)}$. A equação $(2.74)$ nos dá o parâmetro de Hubble $H(z)$ normalizado por $H_{0}$ como

$$
E(z)=\left[\Omega_{r}^{(0)}(1+z)^{4}+\Omega_{m}^{(0)}(1+z)^{3}+\Omega_{d}^{(0)}(1+z)^{3\left(1+\omega_{d}\right)}+\Omega_{K}^{(0)}(1+z)^{2}\right]^{1 / 2} .
$$

Usando a relação $\mathrm{d} t=-\mathrm{d} z /[(1+z) H]$, a idade do universo pode ser expressa como

$$
t_{0}=H_{0}^{-1} \int_{0}^{\infty} \frac{\mathrm{d} z}{E(z)(1+z)}
$$

A integral (4.2) é dominada por termos de baixo redshift. Como $\Omega_{r}^{(0)}$ é de ordem $10^{-5}$, a radiação torna-se importante somente para altos redshifts $(z \gtrsim 1000)$, de modo que podemos desprezar a contribuição da radiação para a integral (4.2). Considerando o caso de uma constante cosmológica $\left(\omega_{d}=-1\right)$, a idade do universo é dada por

$$
t_{0}=H_{0}^{-1} \int_{1}^{\infty} \frac{\mathrm{d} x}{x\left[\Omega_{m}^{(0)} x^{3}+\Omega_{d}^{(0)}+\Omega_{K}^{(0)} x^{2}\right]^{1 / 2}},
$$

onde $x \equiv 1+z$ e $\Omega_{m}^{(0)}+\Omega_{d}^{(0)}+\Omega_{K}^{(0)}=1$.

Para um universo plano $\left(\Omega_{K}^{(0)}=0\right)$, a integral (4.3) pode ser resolvida e obtemos um resultado analítico igual a 


$$
t_{0}=\frac{H_{0}^{-1}}{3 \sqrt{1-\Omega_{m}^{(0)}}} \ln \left(\frac{1+\sqrt{1-\Omega_{m}^{(0)}}}{1-\sqrt{1-\Omega_{m}^{(0)}}}\right)
$$

onde $\Omega_{m}^{(0)}+\Omega_{d}^{(0)}=1$ foi utilizado. No limite $\Omega_{d}^{(0)} \rightarrow 0$, temos que

$$
t_{0}=\frac{2}{3} H_{0}^{-1}
$$

Se utilizarmos $h=0,72 \pm 0,08$, a idade do universo na ausência de uma constante cosmológica é limitada ao intervalo $8,2<t_{0}<10,2$, com o tempo medido em bilhões de anos. A idade dos aglomerados globulares de estrelas mais antigos da Via Láctea é observada como sendo dada aproximadamente por 13, $0 \pm 2$ bilhões de anos [26,27]. Desse modo, vemos que a idade cósmica estimada pela equação (4.5) é inconsistente com as idades dos aglomerados mais antigos da Via Láctea.

Esse problema pode ser solucionado se uma constante cosmológica for levada em consideração. A equação (4.4) mostra que $t_{0}$ torna-se maior para $\Omega_{m}^{(0)}$ decrescente. No limite $\Omega_{m}^{(0)} \rightarrow 0$ temos que $t_{0} \rightarrow \infty$. Para que a condição $t_{0}>11$ bilhões de anos seja respeitada, temos que satisfazer $0<\Omega_{m}^{(0)}<0,55$. A Figura 4.1 ilustra essa condição. Por outro lado, a idade do universo é dada pelas observações das anisotropias da radiação cósmica de fundo feitas pelo satélite WMAP, que assume o modelo $\Lambda$ CDM em suas análises, como sendo $t_{0}=13,772 \pm 0,006$ bilhões de anos [7]. Com esses limites para $t_{0}$, temos que o parâmetro de densidade da matéria não-relativística é limitado a $0,245<\Omega_{m}^{(0)}<0,261$ para $h=0,72$.

\subsubsection{Observações de Supernovas}

A explosão de uma supernova é um evento de intensidade espetacular. Isso faz com que elas sejam extremamente luminosas e, assim, possam ser facilmente catalogadas e estudadas. As supernovas podem ser classificadas de acordo com as linhas de absorção observadas em seu espectro. Se o espectro de uma supernova inclui a linha espectral do hidrogênio, ela é classificada como do tipo II, senão ela é do tipo I. Se a supernova contém a linha de absorção do silício simplesmente ionizado, ela é classificada como do tipo Ia. A explosão de uma supernova tipo Ia ocorre, acredita-se, quando a massa de uma anã branca em um sistema binário ultrapassa o limite de Chandrasekhar ao absorver o gás de sua estrela companheira. Como a luminosidade absoluta de uma supernova tipo Ia é aproximadamente constante no seu pico de luminosidade, a distância de uma supernova Ia pode ser determinada ao observarmos sua luminosidade aparente. Com essas características, as supernovas 


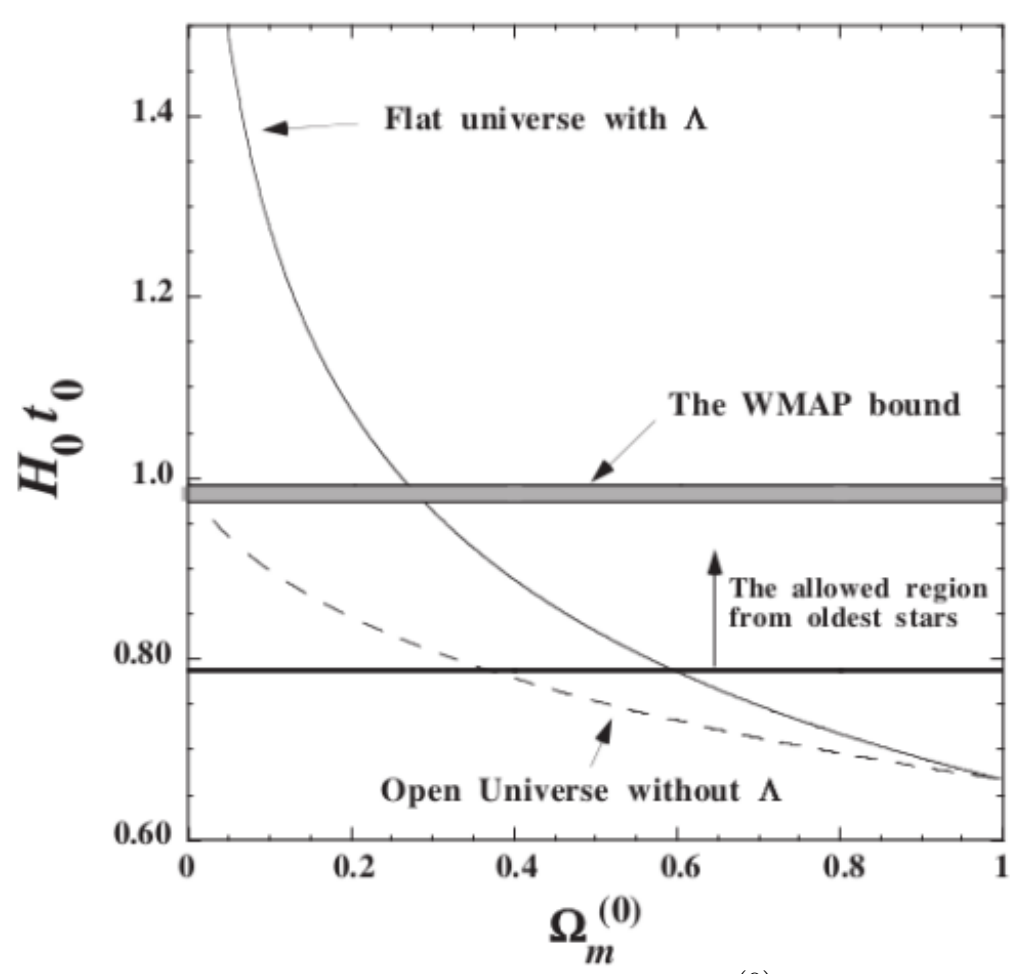

Figura 4.1: Idade cósmica $t_{0}$ em unidades de $H_{0}^{-1}$ versus $\Omega_{m}^{(0)}$. A curva sólida fina descreve o universo plano na presença de uma constante cosmológica com a relação $\Omega_{m}^{(0)}+\Omega_{d}^{(0)}=1$. A curva tracejada corresponde a um universo aberto sem constante cosmológica. A linha sólida grossa é a idade mínima permitida baseado na idade dos aglomerados globulares mais antigos (> 10 bilhões de anos). Também é mostrado o limite vindo dos dados do WMAP com $h=0,70$. O universo plano com constante cosmológica é consistente com o limite do WMAP para $0,271<\Omega_{m}^{(0)}<0,289$ [18].

Ia são um tipo de vela padrão através das quais a distância de luminosidade pode ser medida observacionalmente.

A magnitude aparente, $m$, é geralmente utilizada para medir o brilho das estrelas observadas na Terra. Dois objetos estrelares com fluxos aparentes dados por $\mathcal{F}_{1}$ e $\mathcal{F}_{2}$ possuem suas magnitudes aparentes relacionadas através da expressão

$$
m_{1}-m_{2}=-\frac{5}{2} \log \left(\frac{\mathcal{F}_{1}}{\mathcal{F}_{2}}\right) .
$$

Isso implica que uma estrela com $m_{1}=1$ é 100 vezes mais brilhante que uma com $m_{2}=6$.

A magnitude absoluta $M$ de um objeto é definida em termos da magnitude aparente e da distância de luminosidade $d_{L}$ como

$$
m-M=5 \log \left(\frac{d_{L}}{10 \mathrm{pc}}\right) .
$$

Se a distância for expressa em Megaparsec, então essa relação pode ser escrita como 


$$
m-M=5 \log d_{L}+25 .
$$

A magnitude absoluta das supernovas Ia é conhecida como sendo algo ao redor de $M=-19$ no pico de luminosidade. Se considerarmos duas supernovas Ia cujas magnitudes aparentes são $m_{1}$ e $m_{2}$ e suas distâncias de luminosidade $d_{L_{1}}$ e $d_{L_{2}}$, obtemos através da equação (4.8) a relação

$$
m_{1}-m_{2}=5 \log \left(\frac{d_{L_{1}}}{d_{L_{2}}}\right) .
$$

Consideraremos o caso em que o universo é dominado por um fluido não-relativístico e pela energia escura com uma equação de estado $\omega_{d}=-1$. Nesse caso, o parâmetro de Hubble $H(z)$ é dado pela equação (2.74) com $\Omega_{r}^{(0)} \simeq 0$. Assim, a expressão da distância de luminosidade para um universo plano (2.61) é dada por

$$
d_{L}(z)=\frac{c(1+z)}{H_{0}} \int_{0}^{z} \frac{\mathrm{d} z}{\left[\left(1-\Omega_{d}^{(0)}\right)(1+z)^{3}+\Omega_{d}^{(0)}\right]^{1 / 2}} .
$$

Essa distância luminosa pode ser avaliada numericamente e então comparada com as observações de várias supernovas Ia em diferentes redshifts.

Utilizando 42 supernovas do tipo Ia com redshifts entre 0,18 e 0,83 juntamente com 18 supernovas do tipo Ia com redshifts pequenos, mostrou-se que uma constante cosmológica (ou uma energia escura) está presente no universo em um nível de confiança de $99 \%$ [24,25]. Mostrou-se também que um universo aberto sem constante cosmológica não é compatível com os dados observados. O valor do parâmetro de densidade da matéria não-relativística foi limitada a $\Omega_{m}^{(0)}=0,28 \pm 0,09 \mathrm{em} \mathrm{um}$ universo com uma constante cosmológica. A Figura 4.2 mostra a relação entre dados observacionais de supernovas do tipo Ia e curvas teóricas para a distância de luminosidade. Apesar de novos avanços na compreensão da física das supernovas e de mais supernovas do tipo Ia sendo observadas, o cenário pouco mudou; o estudo da distância de luminosidade de supernovas do tipo Ia mostra elegantemente a necessidade de uma energia escura para explicar os dados observados.

\subsubsection{A Radiação Cósmica de Fundo em Microondas}

A observação das anisotropias na temperatura da radiação cósmica de fundo em microondas (CMB) pode ser utilizada para testar a existência da energia escura. Os fótons mais antigos do universo que podemos observar são oriundos da época conhecida como o último espalhamento. Essa época corresponde ao período em que os elétrons conseguiram ligar-se ao hidrogênio para formar os primeiros átomos 


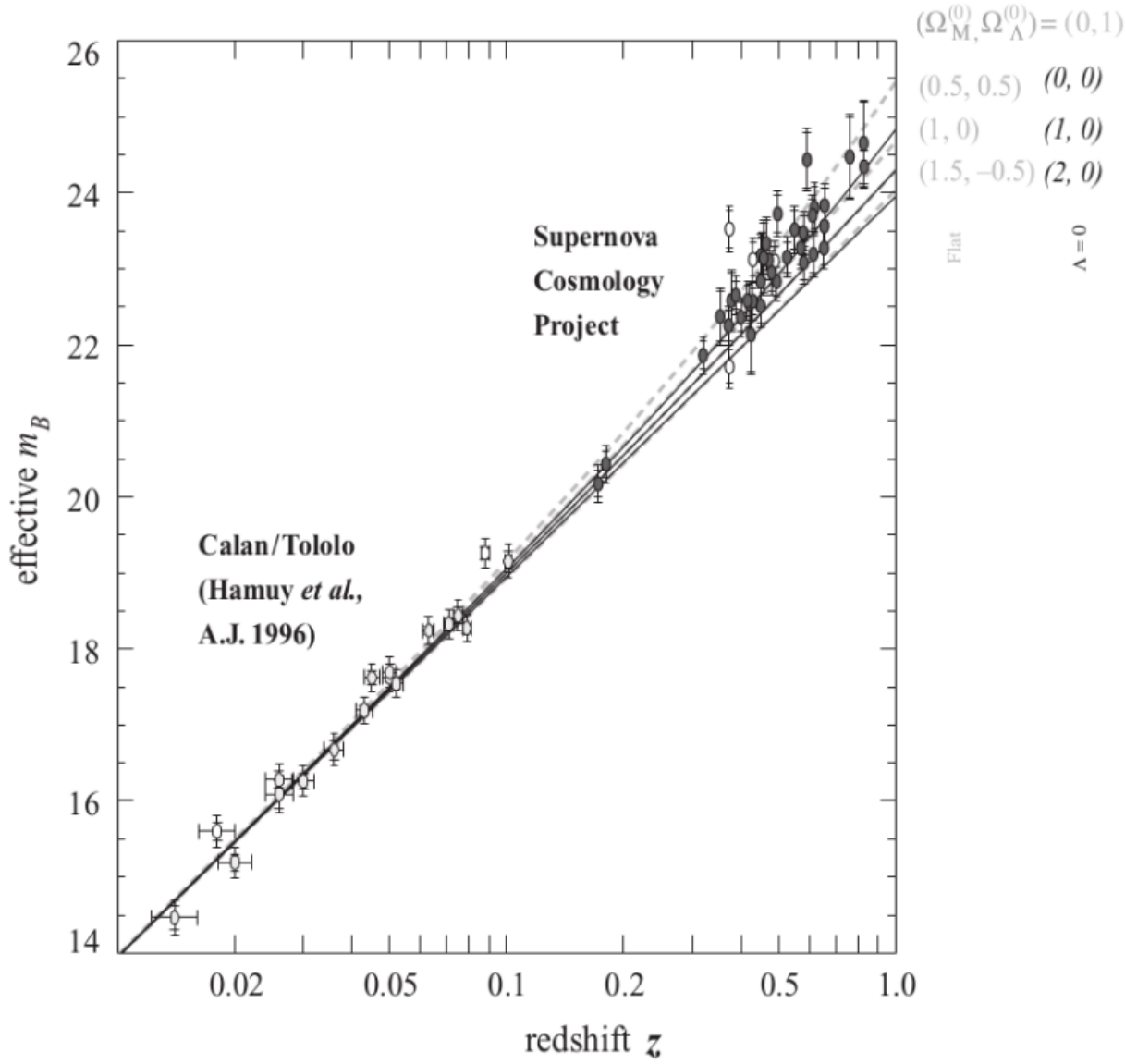

Figura 4.2: A luminosidade efetiva aparente $m_{B}$ versus o redshift para 42 supernova do tipo Ia de alto redshift do Supernova Cosmology Project [24] e 18 supernovas do tipo Ia do Calan/Tololo Supernova Survey [28]. As barras de erro verticais internas e externas representam a incerteza da medida e a incerteza total quando a dispersão de luminosidade da largura da curva de luz correta das supernovas do tipo Ia é adicionada na quadratura, respectivamente. As barras de erro horizontais mostram a incerteza da velocidade peculiar. As curvas sólidas são a previsão teórica para $m_{B}$ para um número de modelos cosmológicos sem constante cosmológica: $\left(\Omega_{m}^{(0)}, \Omega_{\Lambda}^{(0)}\right)=(0,0)$ (topo), (1,0) (meio) e $(2,0)$ (fundo). As linhas tracejadas correspondem a modelos cosmológicos planos: $\left(\Omega_{m}^{(0)}, \Omega_{\Lambda}^{(0)}\right)=(0,1)$ (topo), $(0,5,0,5)$ (segundo), $(1,0)$ (terceiro) e $(1,5,-0,5)$ (fundo) [18].

neutros. Esse processo é conhecido como recombinação e ocorreu ao redor do redshift $z \simeq 1090$. Antes dessa época, os fótons eram fortemente acoplados aos bárions e somente depois da recombinação eles puderam mover-se livremente até nós para que pudéssemos observá-los.

Como vimos anteriormente, a equação em primeira ordem na teoria de perturbações lineares para o monopolo da anisotropia da temperatura $\Theta_{0}$, no regime em que os fótons e os bárions estão fortemente acoplados $\left(\tau_{\mathrm{op}} \gg 1\right)$, é dada por

$$
\Theta_{0}^{\prime \prime}+\frac{R_{s}}{1+R_{s}} \mathcal{H} \Theta_{0}^{\prime}+k^{2} c_{s}^{2} \Theta_{0}=-\frac{k^{2}}{3} \Psi-\frac{R_{s}}{1+R_{s}} \mathcal{H} \Phi^{\prime}-\Phi^{\prime \prime}
$$


onde a velocidade do som é dada por $c_{s}=\sqrt{1 / 3\left(1+R_{s}\right)}$ com a razão entre as densidade dos bárions e a densidade dos fótons sendo dada por $R_{s}=3 \rho_{b} / \rho_{\gamma}$. Essa razão evolui, devido à expansão cósmica, como

$$
R_{s}=\frac{3 \omega_{b}}{4 \omega_{\gamma}} \frac{1}{1+z}
$$

onde definimos

$$
\omega_{b} \equiv \Omega_{b}^{(0)} h^{2}, \quad \omega_{\gamma} \equiv \Omega_{\gamma}^{(0)} h^{2}
$$

Se tomarmos os seguintes valores para a quantidade de fótons e bárions, $\omega_{b}=$ 0,02267 e $\omega_{\gamma}=2,469 \times 10^{-5}$, obtemos que $R_{s}=2,7 \times 10^{4} /(1+z)$, o que é muito menor que a unidade para redshifts maiores que $10^{5}$, época que corresponde ao regime de acoplamento forte entre fótons e bárions.

Tomando a derivada de $R_{s}$ com respeito a $\eta$ e usando a definição de redshift (2.26), obtemos a relação $R_{s}^{\prime}=\mathcal{H} R_{s}$. Assim, a equação (4.11) pode ser escrita como

$$
\left[\frac{\mathrm{d}^{2}}{\mathrm{~d} \eta^{2}}+\frac{R_{s}^{\prime}}{1+R_{s}} \frac{\mathrm{d}}{\mathrm{d} \eta}+k^{2} c_{s}^{2}\right]\left(\Theta_{0}+\Phi\right)=\frac{k^{2}}{3}\left(\frac{1}{1+R_{s}} \Phi-\Psi\right) .
$$

O segundo termo do lado esquerdo da equação (4.14) é da ordem de $R_{s} \eta^{-2}\left(\Theta_{0}+\Phi\right) \approx$ $R_{s} \mathcal{H}^{2}\left(\Theta_{0}+\Phi\right)$, enquanto o terceiro termo é da ordem de $k^{2} c_{s}^{2}\left(\Theta_{0}+\Phi\right)$. Portanto, o segundo termo pode ser desprezado na condição em que $R_{s} \ll c_{s}^{2}(k / \mathcal{H})^{2}$. No regime fortemente acoplado, temos que $R_{s} \ll 1$ e essa condição é satisfeita para comprimentos de onda menores que o raio de Hubble $(k>\mathcal{H})$. Desse modo, a solução homogênea da equação (4.14) pode ser descrita pela soma de soluções do tipo $\exp \left( \pm i \int k c_{s} \mathrm{~d} \eta\right)$, isto é,

$$
\left(\Theta_{0}+\Phi\right)^{\operatorname{hom}}(k, \eta)=c_{1} f_{1}(k, \eta)+c_{s} f_{2}(k, \eta)
$$

onde $f_{1}(\eta)=\sin \left[k r_{s}(\eta)\right], f_{2}(\eta)=\cos \left[k r_{s}(\eta)\right]$ e $r_{s}$ é o horizonte sonoro definido por

$$
r_{s}(\eta) \equiv \int_{0}^{\eta} \mathrm{d} \eta c_{s}(\eta)
$$

Podemos ver claramente com a equação (4.15) que a solução homogênea leva a oscilações nas anisotropias da CMB com uma frequência dependente do tempo $\omega_{k}(\eta)=k r_{s}(\eta)$.

A solução geral da equação (4.14) pode ser obtida pelo método da função de Green $[8,18]$. No regime fortemente acoplado $\left(R_{s} \ll 1\right)$, ela é dada por 


$$
\begin{aligned}
\left(\Theta_{0}+\Phi\right)(k, \eta) & =c_{1} f_{1}(\eta)+c_{2} f_{2}(\eta) \\
& +\frac{k^{2}}{3} \int_{0}^{\eta} \mathrm{d} \tilde{\eta}[\Phi(\tilde{\eta})-\Psi(\tilde{\eta})] \frac{f_{1}(\tilde{\eta}) f_{2}(\eta)-f_{1}(\eta) f_{2}(\tilde{\eta})}{f_{1}(\tilde{\eta}) f_{2}^{\prime}(\tilde{\eta})-f_{1}^{\prime}(\tilde{\eta}) f_{2}(\tilde{\eta})}
\end{aligned}
$$

Tomando as condições iniciais como sendo $\Theta_{0}^{\prime}=0$ e $\Phi^{\prime}=0$, os coeficientes $c_{1}$ e $c_{2}$ podem ser determinados. Assim, a equação (4.17) reduz-se a

$$
\begin{aligned}
\left(\Theta_{0}+\Phi\right)(k, \eta) & =\left[\Theta_{0}(0)+\Phi(0)\right] \cos \left(k r_{s}\right) \\
& +\frac{k}{\sqrt{3}} \int_{0}^{\eta} \mathrm{d} \tilde{\eta}[\Phi(\tilde{\eta})-\Psi(\tilde{\eta})] \sin \left[k\left(r_{s}(\eta)-r_{s}(\tilde{\eta})\right)\right]
\end{aligned}
$$

onde a aproximação $k c_{s}(\eta) \simeq k / \sqrt{3}$ foi utilizada. Usando as equações (3.138) e (4.18), a solução para o momento de dipolo é dada por $[8,18]$

$$
\begin{aligned}
\Theta_{1}(k, \eta) & =\frac{1}{\sqrt{3}}\left[\Theta_{0}(0)+\Phi(0)\right] \sin \left(k r_{s}\right) \\
& -\frac{k}{3} \int_{0}^{\eta} \mathrm{d} \tilde{\eta}[\Phi(\tilde{\eta})-\Psi(\tilde{\eta})] \cos \left[k\left(r_{s}(\eta)-r_{s}(\tilde{\eta})\right)\right] .
\end{aligned}
$$

O primeiro termo do lado direito da equação (4.18) mostra que existe um pico para as soluções homogêneas de $\Theta_{0}+\Phi$ nas posições que satisfazem a relação

$$
k r_{s}=n \pi,
$$

onde $n$ são números inteiros. Essa equação é válida somente como uma aproximação para a posição dos picos, visto que a posição dos picos é sujeita a correções devido à presença do último termo na equação (4.18). Além disso, temos que a solução do dipolo (4.19) também contribui para o espectro da CMB.

Para obtermos as anisotropias da CMB observadas hoje, precisamos estudar a evolução das perturbações depois que os fótons começaram a propagar-se livremente até que fossem observados por nós. Nessa situação, contribuições dos momentos de ordem superiores $\left(\Theta_{\ell}\right.$ com $\left.\ell \geq 2\right)$ e o efeito de Sachs-Wolfe integrado devem ser considerados. A solução para as anisotropias observadas pode ser escrita na forma semi-analítica [8], 


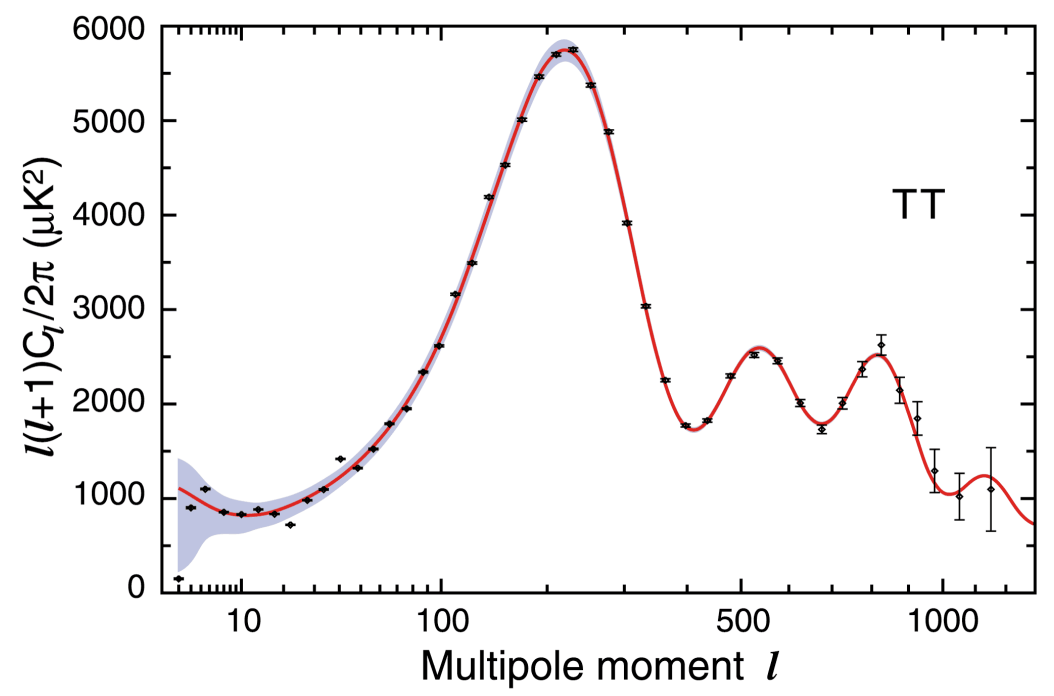

Figura 4.3: $\mathrm{O}$ espectro de potência da $\mathrm{CMB}, \ell(\ell+1) C_{\ell} / 2 \pi$, versus o momento do multipolo $\ell$. A curva mostra a previsão teórica para o espectro de potência, enquanto os pontos representam os dados do WMAP [7].

$$
\begin{aligned}
\Theta_{\ell}\left(k, \eta_{0}\right) & \simeq\left[\Theta_{0}\left(k, \eta_{*}\right)+\Psi\left(k, \eta_{*}\right)\right] j_{\ell}\left[k\left(\eta_{0}-\eta_{*}\right)\right] \\
& +3 \Theta_{1}\left(k, \eta_{*}\right)\left[j_{\ell-1}\left[k\left(\eta_{0}-\eta_{*}\right)\right]-\frac{(\ell+1) j_{\ell}\left[k\left(\eta_{0}-\eta_{*}\right)\right]}{k\left(\eta_{0}-\eta_{*}\right)}\right] \\
& +\int_{0}^{\eta_{0}} \mathrm{~d} \eta e^{-\tau_{\mathrm{op}}}\left[\Psi^{\prime}(k, \eta)-\Phi^{\prime}(k, \eta)\right] j_{\ell}\left[k\left(\eta_{0}-\eta\right)\right],
\end{aligned}
$$

onde $j_{\ell}(x)$ é a função esférica de Bessel e $\eta_{*}$ é o tempo conforme que corresponde ao pico da função de visibilidade definida como [8]

$$
g(\eta) \equiv-\tau_{\mathrm{op}}^{\prime} e^{-\tau_{\mathrm{op}}}
$$

A função de visibilidade quantifica a probabilidade de que a última interação entre fótons e elétrons ocorreu em $\eta$. Ela obedece a condição de normalização $\int_{0}^{\eta_{0}} \mathrm{~d} \eta g(\eta)=$ 1. Antes da época da recombinação (quando a profundidade ótica $\tau_{\mathrm{op}}$ era muito maior que 1), a função de visibilidade é aproximadamente zero. Ela tem um pico ao redor de $z \sim 1000$. A taxa de espalhamento, $-\tau_{\text {op }}^{\prime}$ (ver equação (3.130)), decresce rapidamente depois da recombinação, visto que os fótons param de interagir com os elétrons e os prótons livres, e assim $g(\eta)$ aproxima-se de zero novamente.

A solução analítica (4.21) reproduz soluções numéricas com $10 \%$ de acurácia, o que para os atuais níveis de precisão das observações cosmológicas não é suficiente, mas ainda assim é esclarecedora em relação à física por trás das anisotropias da CMB. O primeiro termo do lado direito da equação (4.21) é oriundo da inte- 
gral $\int_{0}^{\eta_{0}} \mathrm{~d} \eta g(\eta)\left[\Theta_{0}(k, \eta)+\Psi(k, \eta)\right] j_{\ell}\left[k\left(\eta-\eta_{0}\right)\right]$. Esse termo carrega a informação da solução de monopolo (4.18). O efeito da posterior propagação livre dos fótons aparece na função de visibilidade $g(\eta)$, cuja principal contribuição é dada por seu valor ao redor de $\eta=\eta_{*}$. A posição dos picos estimados pela equação (4.20) é deslocada para valores maiores de $k$ devido à propagação livre dos fótons. O segundo termo da equação (4.21) surge da contribuição do dipolo $\Theta_{1}$, que também leva a modificações no espectro da CMB. O terceiro termo na equação (4.21) é responsável pelo efeito Sachs-Wolfe integrado, que é induzido pela variação dos potenciais gravitacionais $\Psi$ e $\Phi$. Os potenciais $\Psi$ e $\Phi$ mantém-se constantes durante a época dominada pela matéria, entretanto, quando a energia escura torna-se dominante, esses potenciais variam substancialmente.

Para podermos confrontar as anisotropias da temperatura da CMB previstas pela teoria com as observações feitas, temos que expandir a perturbação $\Theta$ em função dos esféricos harmônicos [10],

$$
\Theta(\mathbf{x}, \eta)=\sum_{\ell=1}^{\infty} \sum_{m=-\ell}^{\ell} a_{\ell m}(\mathbf{x}, \eta) Y_{\ell m}(\hat{n}),
$$

onde os subscritos $\ell$ e $m$ são parâmetros conjugados ao vetor unitário $\hat{n}$ que representa a direção dos fótons que se propagam na direção do observador. Os esféricos harmônicos $Y_{\ell m}$ satisfazem a condição de normalização

$$
\int \mathrm{d} \Omega Y_{\ell m}(\hat{n}) Y_{\ell^{\prime} m^{\prime}}^{*}(\hat{n})=\delta_{\ell \ell^{\prime}} \delta_{m m^{\prime}}
$$

Os coeficientes na equação (4.23) podem ser considerados estatisticamente independentes [10]. Isso significa que o valor médio de $a_{\ell m}$ é zero $\left(\left\langle a_{\ell m}\right\rangle=0\right)$ com a variância dada por

$$
C_{\ell} \equiv\left\langle\left|a_{\ell m}\right|^{2}\right\rangle
$$

A variância $C_{\ell}$ pode ser expressa em termos dos momentos da temperatura $\Theta_{\ell}(k)$ através da expressão

$$
C_{\ell}=\frac{2}{\pi} \int_{0}^{\infty} \mathrm{d} k k^{2}\left|\Theta_{\ell}(k)\right|^{2}
$$

A equação (4.20) mostra que o comprimento de onda comóvel correspondente aos picos acústicos da CMB podem ser aproximados como $\lambda_{c}=2 \pi / k=(2 / n) r_{s}$. Podemos, então, definir o ângulo característico para a localização dos picos como sendo dado por 


$$
\theta_{A} \equiv \frac{r_{s}\left(z_{\mathrm{rec}}\right)}{d_{A}^{(c)}\left(z_{\mathrm{rec}}\right)}
$$

onde $z_{\text {rec }}$ é o redshift da época da recombinação e $d_{A}^{(c)}$ é a distância de diâmetro angular comóvel definida como

$$
d_{A}^{(c)}(z) \equiv \frac{d_{A}(z)}{a}=(1+z) d_{A}(z) .
$$

A distância de diâmetro angular física é definida pela equação (2.64). O multipolo $\ell$ correspondente ao ângulo (4.27) é

$$
\ell_{A}=\frac{\pi}{\theta_{A}}=\pi \frac{d_{A}^{(c)}\left(z_{\mathrm{rec}}\right)}{r_{s}\left(z_{\mathrm{rec}}\right)}
$$

Das equações $(2.64)$ e (4.28), a distância de diâmetro angular comóvel $d_{A}^{(c)}\left(z_{\text {rec }}\right)$ é dada por

$$
d_{A}^{(c)}\left(z_{\mathrm{rec}}\right)=\frac{c}{H_{0}} \frac{1}{\sqrt{\Omega_{m}^{(0)}}} \mathcal{R},
$$

onde $\mathcal{R}$ é chamado de parâmetro de deslocamento da CMB e é definido por $[18,29]$

$$
\mathcal{R}=\sqrt{\frac{\Omega_{m}^{(0)}}{\Omega_{K}^{(0)}}} \sinh \left(\sqrt{\Omega_{K}^{(0)}} \int_{0}^{z_{\mathrm{rec}}} \frac{\mathrm{d} z}{E(z)}\right) .
$$

Das equações $(3.142)$ e (4.16), o horizonte sonoro $r_{s}\left(z_{\text {rec }}\right)$ é

$$
r_{s}\left(z_{\mathrm{rec}}\right)=\frac{c}{\sqrt{3} a_{0} H_{0}} \int_{z_{\mathrm{rec}}}^{\infty} \frac{\mathrm{d} z}{\sqrt{1+R_{s}} E(z)},
$$

onde o redshift $z_{\text {rec }}$ é aproximadamente igual a 1090.

Como a contribuição da energia escura para $E(z)$ na equação (4.32) é desprezível para $z>z_{\text {rec }}$, podemos estimar essa quantidade como $E(a)=\left(\frac{\sqrt{a+a_{\mathrm{eq}}}}{a^{2}}\right) \sqrt{\Omega_{m}^{(0)}}$, onde $a_{\mathrm{eq}}=\left(1+z_{\mathrm{eq}}\right)^{-1}$ é o fator de escala na época da igualdade entre matéria e radiação. Assim, a integral (4.32) pode ser escrita como

$$
r_{s}\left(z_{\mathrm{rec}}\right)=\frac{c}{\sqrt{3} H_{0}} \frac{1}{\sqrt{\Omega_{m}^{(0)}}} \int_{0}^{a_{\mathrm{rec}}} \frac{1}{\sqrt{1+R_{s}(a)}} \frac{1}{\sqrt{a+a_{\mathrm{eq}}}} \mathrm{d} a
$$

onde

$$
R_{s}(a)=\left(3 \omega_{b} / 4 \omega_{\gamma}\right) a
$$




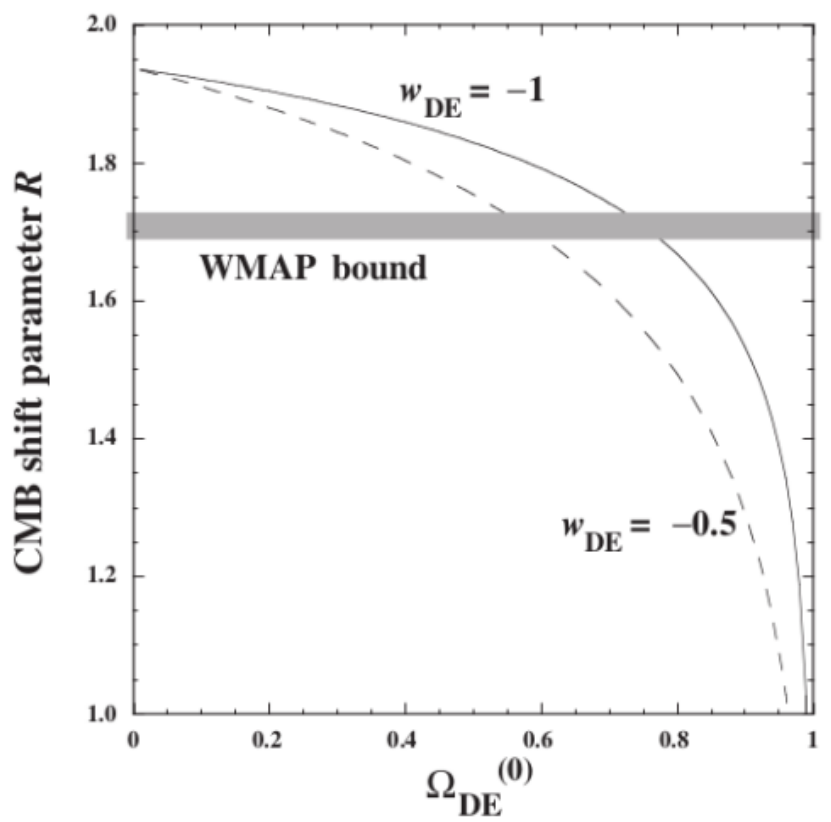

Figura 4.4: O parâmetro de deslocamento da CMB $\mathcal{R}$ versus $\Omega_{d}^{(0)}$ para $\omega_{d}=-1$ e $\omega_{d}=$ $-0,5$ em um universo plano. Temos também o limite vindo dos dados do WMAP. Para a constante cosmológica $\left(\omega_{d}=-1\right)$, o limite observacional para $\Omega_{d}^{(0)}$ vindo do parâmetro de deslocamento da CMB é $0,72<\Omega_{d}^{(0)}<0,77$ [18].

A equação (4.33) pode ser integrada, sendo o resultado obtido dado por

$$
r_{s}\left(z_{\mathrm{rec}}\right)=\frac{4}{3} \frac{c h}{H_{0}} \sqrt{\frac{\omega_{\gamma}}{\omega_{m} \omega_{b}}} \ln \left(\frac{\sqrt{R_{s}^{(\mathrm{rec})}+R_{s}^{(\mathrm{eq})}}+\sqrt{1+R_{s}^{(\mathrm{rec})}}}{1+\sqrt{R_{s}^{(\mathrm{eq})}}}\right),
$$

onde $R_{s}^{(\mathrm{rec})}=R_{s}\left(a_{\mathrm{rec}}\right)$ e $R_{s}^{(\mathrm{eq})}=R_{s}\left(a_{\mathrm{eq}}\right)$.

Das equações (4.30) e (4.35), o multipolo $\ell_{A}$ na equação (4.29) é

$$
\ell_{A}=\frac{3 \pi}{4} \sqrt{\frac{\omega_{b}}{\omega_{\gamma}}} \mathcal{R}\left[\ln \left(\frac{\sqrt{R_{s}^{(\mathrm{rec})}+R_{s}^{(\mathrm{eq})}}+\sqrt{1+R_{s}^{(\mathrm{rec})}}}{1+\sqrt{R_{s}^{(\mathrm{eq})}}}\right)\right]^{-1} .
$$

Essa expressão mostra que $\ell_{A}$ é proporcional a $\mathcal{R}$. O parâmetro de deslocamento da CMB definido pela equação (4.31) depende da expansão cósmica desde a recombinação até o presente. Durante esse período a contribuição da energia escura torna-se cada vez mais importante. Portanto, o parâmetro de deslocamento da CMB pode ser utilizado para determinar os parâmetros da energia escura. Segundo os resultados do WMAP, $\mathcal{R}$ é limitado a [7]

$$
\mathcal{R}=1,70 \pm 0,019
$$


Quando utilizamos uma constante cosmológica com $\omega_{d}=-1$ e consideramos um universo plano $\Omega_{K}^{(0)}=0$, o parâmetro de densidade da energia escura é limitado a $0,72<\Omega_{d}^{(0)}<0,77$ pelo parâmetro de deslocamento dado por (4.37). A Figura 4.4 mostra a dependência do parâmetro de deslocamento com o parâmetro de densidade da energia escura. Isso faz da análise das anisotropias da CMB outra observação que indica a existência de uma energia escura dominante no universo atual.

\subsection{Constante Cosmológica}

O candidato mais simples para a energia escura é a constante cosmológica $\Lambda$, que possui densidade de energia constante no tempo e no espaço. Na atual conjuntura observacional, o modelo $\Lambda$ CDM é totalmente consistente com as observações realizadas. A densidade lagrangiana para o modelo $\Lambda$ CDM é dada simplesmente por um termo linear em $R$ mais uma constante $\Lambda$. Apesar de sua atraente simplicidade, é consideravelmente difícil explicar o porquê da escala de energia da constante cosmológica requerida pela aceleração cósmica do universo atual ser muito menor do que o que é previsto pela física de partículas para a energia do vácuo. A densidade de energia do vácuo calculada ao somarmos a energia de ponto-zero do campo escalar é aproximadamente $10^{121}$ de vezes maior que a densidade de energia observada para a energia escura [30].

O problema de um valor desconcertantemente grande para $\Lambda$ é independente da observação da aceleração cósmica do atual universo. Mesmo sem nenhuma evidência observacional para a energia escura, ainda seria necessária uma explicação para que a constante cosmológica seja igual a zero. Os modelos para a energia escura alternativos ao $\Lambda \mathrm{CDM}$ são baseados na suposição de que $\Lambda$ é zero ou desprezível. Assim, o problema da constante cosmológica é achar um mecanismo que a faça igual a zero ou a faça desprezível em relação à densidade cosmológica atual, sendo que, nesse cenário, uma outra fonte para a expansão do universo precisa ser proposta.

\subsubsection{Equações de Einstein com uma Constante Cosmológica}

O tensor de energia-momento $T_{\mu \nu}$ do lado direito das equações de Einstein (2.8) obedece a lei de conservação $\nabla^{\nu} T_{\mu \nu}=0$. Como a métrica $g_{\mu \nu}$ satisfaz a relação $\nabla^{\nu} g_{\mu \nu}=0$, é possível adicionar o termo $\Lambda g_{\mu \nu}$ nas equações de Einstein sem desrespeitar a lei de conservação,

$$
R_{\mu \nu}-\frac{1}{2} g_{\mu \nu} R+\Lambda g_{\mu \nu}=8 \pi G T_{\mu \nu}
$$


É interessante notar que essas equações são as equações de segunda ordem na métrica em quatro dimensões mais gerais que podemos construir, resultado conhecido como Teorema de Lovelock [6,31].

As equações de Einstein (4.38) podem ser derivadas através do princípio da ação. A ação, nesse caso, é linear no escalar de Ricci, $R=g^{\mu \nu} R_{\mu \nu}$, e a ação total para o sistema é a ação geométrica somada à ação da matéria,

$$
S=\frac{1}{16 \pi G} \int \mathrm{d}^{4} x \sqrt{-g}(R-2 \Lambda)+S_{m} .
$$

A variação da ação (4.39) com respeito a $g^{\mu \nu}$ nos leva a [6]

$$
\begin{aligned}
& \delta S=\frac{1}{16 \pi G} \int \mathrm{d}^{4} x[ \delta(\sqrt{-g})\left(g^{\mu \nu} R_{\mu \nu}-2 \Lambda\right) \\
&\left.+\sqrt{-g} \delta g^{\mu \nu} R_{\mu \nu}+\sqrt{-g} g^{\mu \nu} \delta R_{\mu \nu}\right]+\delta S_{m} .
\end{aligned}
$$

Como $\delta R_{\mu \nu}=\left(\delta \Gamma_{\mu \nu}^{\alpha}\right)_{; \alpha}-\left(\delta \Gamma_{\mu \alpha}^{\alpha}\right)_{; \nu}$, temos que $g^{\mu \nu} \delta R_{\mu \nu}=\left(g^{\mu \nu} \delta \Gamma_{\mu \nu}^{\alpha}-g^{\mu \alpha} \delta \Gamma_{\mu \nu}^{\nu}\right)_{; \alpha}$ portanto,

$$
\int \mathrm{d}^{4} x \sqrt{-g} g^{\mu \nu} \delta R_{\mu \nu}=\int \mathrm{d}^{4} x \sqrt{-g}\left(g^{\mu \nu} \delta \Gamma_{\mu \nu}^{\alpha}-g^{\mu \alpha} \delta \Gamma_{\mu \nu}^{\nu}\right)_{; \alpha}=0
$$

onde utilizamos o teorema de Gauss na última igualdade. Temos também que $\delta(\sqrt{-g})=-(1 / 2) \sqrt{-g} g_{\mu \nu} \delta g^{\mu \nu}$. Assim, a equação (4.40) torna-se

$$
\delta S=\frac{1}{16 \pi G} \int \mathrm{d}^{4} x \sqrt{-g}\left(R_{\mu \nu}-\frac{1}{2} R g_{\mu \nu}+\Lambda g_{\mu \nu}\right) \delta g^{\mu \nu}+\delta S_{m}
$$

O tensor de energia-momento $T_{\mu \nu}$ é definido como [6]

$$
\delta S_{m}=-\frac{1}{2} \int \mathrm{d}^{4} x \sqrt{-g} T_{\mu \nu} \delta g^{\mu \nu} .
$$

Assim, a equação (4.42) reduz-se a

$$
\delta S=\frac{1}{16 \pi G} \int \mathrm{d}^{4} x \sqrt{-g}\left(R_{\mu \nu}-\frac{1}{2} R g_{\mu \nu}+\Lambda g_{\mu \nu}-8 \pi G T_{\mu \nu}\right) \delta g^{\mu \nu}
$$

As equações de Einstein (4.38) seguem do princípio da ação, $\delta S=0$. Para a métrica de FRW, (2.1), as equações de Einstein (4.38) são dadas por

$$
H^{2}=\frac{8 \pi G}{3} \rho-\frac{K}{a^{2}}+\frac{\Lambda}{3}
$$




$$
\frac{\ddot{a}}{a}=-\frac{4 \pi G}{3}(\rho+3 p)+\frac{\Lambda}{3}
$$

\subsubsection{O Problema do Ajuste Fino}

Podemos ver através da equação (4.45) que, para termos a aceleração cósmica presente, precisamos que a constante cosmológica $\Lambda$ seja da ordem do quadrado do constante de Hubble $H_{0}$ :

$$
\Lambda \approx H_{0}^{2}=\left(2,1332 h \times 10^{-42} \mathrm{GeV}\right)^{2} .
$$

Se interpretarmos essa quantidade como uma densidade de energia, temos que

$$
\rho_{\Lambda} \approx \frac{\Lambda m_{\mathrm{pl}}^{2}}{8 \pi} \approx 10^{-47} \mathrm{GeV}^{4} \approx 10^{-123} \mathrm{~m}_{\mathrm{pl}}^{4}
$$

onde usamos que $h \approx 0,70$ e $m_{\mathrm{pl}}=1 / \sqrt{G} \approx 10^{19} \mathrm{GeV}$.

Suponhamos agora que a densidade de energia (4.48) venha da energia do vácuo $\langle\rho\rangle$ do espaço vazio - uma energia que, a não ser que uma simetria ou um mecanismo físico desconhecido torne zero, deve ser diferente de zero. A energia de ponto-zero de algum campo de massa $m$ com momento $k$ e frequência $\omega$ é dada por $E=\omega / 2=$ $\sqrt{k^{2}+m^{2}} / 2$ (em unidades em que $\hbar=c=1$ ). Somando todas as energias de pontozero desse campo em diferentes escalas de momento $k$, de zero até uma escala de corte arbitrária $k_{\max }(\gg m)$, obtemos que a densidade de energia do vácuo é

$$
\rho_{\mathrm{vac}}=\int_{0}^{k_{\max }} \frac{\mathrm{d}^{3} k}{(2 \pi)^{3}} \frac{1}{2} \sqrt{k^{2}+m^{2}} .
$$

Como essa integral é dominada pelos modos com grande $k(\gg m)$, temos que

$$
\rho_{\mathrm{vac}}=\int_{0}^{k_{\max }} \frac{4 \pi k^{2} \mathrm{~d} k}{(2 \pi)^{3}} \frac{1}{2} \sqrt{k^{2}+m^{2}} \approx \frac{k_{\max }^{4}}{16 \pi^{2}} .
$$

Como a relatividade geral é supostamente válida até a escala de Planck, $m_{\mathrm{pl}}$, tomaremos a escala de corte como $m_{\mathrm{pl}}$, de modo que a densidade de energia do vácuo é estimada como

$$
\rho_{\mathrm{vac}} \simeq 10^{74} \mathrm{GeV}^{4}
$$

Esse valor é aproximadamente $10^{121}$ vezes maior que o valor observado (4.48). Mesmo que outras escalas de energia oriundas da física de partículas sejam tomadas como a escala de corte, essa situação não muda muito. Para a escala de energia da cromodinâmica quântica, $k_{\max } \approx 0,1 \mathrm{GeV}$, temos que $\rho_{\mathrm{vac}} \approx 10^{-3} \mathrm{GeV}^{4}$, o que ainda 
é muito superior a $\rho_{\Lambda}$.

\subsubsection{O Problema da Coincidência}

Outro problema que temos quando a constante cosmológica é considerada como a fonte para a energia escura é que o seu valor é praticamente igual ao valor presente da densidade de energia da matéria. A densidade da matéria, $\rho_{m}=\rho_{m}^{(0)}(1+z)^{3}$, coincide com a densidade cosmológica, $\rho_{\Lambda}^{(0)}$, no redshift

$$
z_{\text {coinc }}=\left(\frac{\Omega_{\Lambda}^{(0)}}{1-\Omega_{\Lambda}^{(0)}}\right)^{1 / 3}-1
$$

que, para $\Omega_{\Lambda}^{(0)}=0,7$, corresponde a $z_{\text {coinc }} \approx 0,3$. Esse problema é conhecido como o problema da coincidência.

Entretanto, o problema da coincidência não é específico da constante cosmológica. Praticamente todos os modelos aceitáveis para a energia escura comportam-se de maneira similar à constante cosmológica e o redshift $z_{\text {coinc }}$ também é próximo de zero. Assim, podemos discutir esse problema em termos de uma densidade de energia escura geral $\rho_{d}$.

Excluindo o caso em que esta questão nada mais é do uma que fortuita coincidência ou que toda evidência observacional em favor da aceleração cósmica esteja sistematicamente errada, vários caminhos foram propostos para aliviar essa coincidência. O primeiro tipo de explicação consiste em modelos em que $\rho_{d}$ é sensível a $\rho_{m}$ e independentemente das condições iniciais sua densidade de energia será da mesma magnitude da densidade da matéria em tempos tardios. Nesse caso, $\Omega_{d}$ é diferente de zero por um período considerável e isso alivia o problema da coincidência. Porém, a aceleração é recente e a coincidência aparece novamente, mas, digamos, de uma maneira menos brusca.

O segundo tipo de explicação diz que não existe coincidência e que $\Omega_{m}$ e $\Omega_{d}$ sempre foram, ou pelo menos foram durante a maior parte do tempo, similares. Em princípio, isso não é difícil de ser alcançado. É suficiente postular dois componentes, um que se aglomera e outro que não se aglomera porque sua velocidade do som é muito grande. Feito isso, basta regular suas equações de estado de forma que suas densidades de energia sempre foram similares. O principal problema nesse cenário é que ou a equação de estado comum sempre satisfaz a condição para aceleração cósmica sendo, portanto, difícil ou impossível de ser consistente com as observações, ou a equação de estado muda exatamente quando necessário, isto é, hoje, de forma que uma nova coincidência aparece. Modelos desse tipo são, por exemplo, os modelos 
conhecidos como scaling.

Um terceiro tipo de explicação invoca o princípio antrópico. De acordo com esse princípio, nós vivemos em um universo com $\rho_{d} \approx \rho_{m}$, porque essa é a maior densidade de energia escura permitida pelo requisito fundamental de que a formação de estruturas no universo seja grande o suficiente para sustentar vida e, em geral, altos valores da energia do vácuo são mais prováveis do que os menores [32].

\subsection{Quintessência}

No modelo padrão da física de partículas, a fauna de campos escalares disponíveis é pequena, contudo, em modelos que tentam ir além do modelo padrão, o número de campos escalares disponíveis aumenta consideravelmente, como no caso da teoria das cordas e da supergravidade [16]. O termo quintessência [33] é utilizado para denotar um campo escalar $\phi$ com um potencial $V(\phi)$ que interage com todos os outros componentes do universo somente através da gravidade e que, diferentemente da constante cosmológica, possui uma equação de estado dinâmica. O modelo da quintessência é, portanto, descrito pela ação

$$
S=\int \mathrm{d}^{4} x \sqrt{-g}\left(\frac{1}{2 \kappa^{2}} R+\mathcal{L}_{\phi}\right)+S_{M}, \quad \mathcal{L}_{\phi}=-\frac{1}{2} g^{\mu \nu} \partial_{\mu} \phi \partial_{\nu} \phi-V(\phi),
$$

onde $\kappa^{2}=8 \pi G, R$ é o escalar de Ricci e $S_{M}$ é a ação da matéria.

Consideraremos que um fluido perfeito com densidade de energia $\rho_{M}$, pressão $P_{M}$ e equação de estado $\omega_{M}=P_{M} / \rho_{M}$ coexiste com a quintessência. Aqui, o subscrito $M$ é utilizado para um fluido perfeito geral. Esse fluido satisfaz a equação de continuidade (2.18), isto é,

$$
\dot{\rho}_{M}+3 H\left(\rho_{M}+P_{M}\right)=0 .
$$

Podemos, através da ação (4.53), facilmente encontrar o tensor de energia-momento da quintessência, que é dado por $[16,18]$

$$
\begin{aligned}
T_{\mu \nu}^{(\phi)} & =-\frac{2}{\sqrt{-g}} \frac{\delta\left(\sqrt{-g} \mathcal{L}_{\phi}\right)}{\delta g^{\mu \nu}} \\
& =\partial_{\mu} \phi \partial_{\nu} \phi-g_{\mu \nu}\left[\frac{1}{2} g^{\alpha \beta} \partial_{\alpha} \phi \partial_{\beta} \phi+V(\phi)\right] .
\end{aligned}
$$

Na métrica de FRW, a densidade de energia $\rho_{\phi}$ e a pressão $P_{\phi}$ do campo escalar são 


$$
\rho_{\phi}=-T_{0}^{0(\phi)}=\frac{1}{2} \dot{\phi}^{2}+V(\phi), \quad P_{\phi}=\frac{1}{3} T_{i}^{i(\phi)}=\frac{1}{2} \dot{\phi}^{2}-V(\phi) .
$$

A equação de estado, então, é dada por

$$
\omega_{\phi} \equiv \frac{P_{\phi}}{\rho_{\phi}}=\frac{\dot{\phi}^{2}-2 V(\phi)}{\dot{\phi}^{2}+2 V(\phi)} .
$$

Em um universo plano, as equações de movimento que seguem das equações de Friedmann são

$$
\begin{gathered}
H^{2}=\frac{\kappa^{2}}{3}\left[\frac{1}{2} \dot{\phi}^{2}+V(\phi)+\rho_{M}\right], \\
\dot{H}=-\frac{\kappa^{2}}{2}\left(\dot{\phi}^{2}+\rho_{M}+P_{M}\right) .
\end{gathered}
$$

A variação da ação (4.53) com respeito a $\phi$ determina a equação de movimento do campo escalar, que é dada por

$$
\ddot{\phi}+3 H \dot{\phi}+V_{, \phi}=0
$$

onde $V_{, \phi} \equiv \mathrm{d} V / \mathrm{d} \phi$.

Durante as épocas dominadas pela matéria ou pela radiação, a densidade de energia $\rho_{M}$ do fluido domina a dinâmica do universo comparada com a influência da densidade da quintessência para essa dinâmica, isto é, $\rho_{M} \gg \rho_{\phi}$. Uma característica desejada para nosso sistema é que $\rho_{\phi}$ siga $\rho_{M}$, de modo que a densidade da energia escura domine em tempos tardios. Se esse comportamento, chamado de tracking, realmente ocorrerá, dependerá da forma do potencial $V(\phi)$.

Para termos aceleração cósmica em tempos tardios, precisamos que a condição $\omega_{\phi}<-1 / 3$ seja respeitada, o que nos leva à condição $\dot{\phi}^{2}<V(\phi)$. Assim, o potencial escalar precisa ser plano o suficiente para que o campo evolua vagarosamente no tempo através do potencial. Essa situação é semelhante à encontrada na inflação cosmológica, de modo que é conveniente introduzir os parâmetros de slow-roll [34]

$$
\epsilon_{s} \equiv \frac{1}{2 \kappa^{2}}\left(\frac{V_{, \phi}}{V}\right)^{2}, \quad \eta_{s} \equiv \frac{V_{, \phi \phi}}{\kappa^{2} V}
$$

Se as condições $\epsilon_{s} \ll 1$ e $\left|\eta_{s}\right| \ll 1$ forem satisfeitas, a evolução do campo escalar é suficientemente lenta para que $\dot{\phi}^{2} \ll V(\phi)$ e $|\ddot{\phi}| \ll|3 H \dot{\phi}|$ nas equações (4.58) e (4.60).

Das equações (4.57) e (4.60), temos que o desvio de $\omega_{\phi}$ de -1 é dado por 


$$
1+\omega_{\phi}=\frac{V_{, \phi}^{2}}{9 H^{2}\left(\xi_{s}+1\right)^{2} \rho_{\phi}},
$$

onde $\xi_{s} \equiv \ddot{\phi} /(3 H \dot{\phi})$. Isso mostra que $\omega_{\phi}$ é sempre maior que -1 para um potencial positivo. No limite do slow-roll, $\left|\xi_{s}\right| \ll 1$ e $\dot{\phi}^{2} / 2 \ll V(\phi)$, temos que $1+\omega_{\phi} \simeq 2 \epsilon_{s} / 3$ se desprezarmos o fluido da matéria na equação (4.58), isto é, $3 H^{2} \simeq \kappa^{2} V(\phi)$, situação válida no universo tardio.

Vários modelos para o potencial da quintessência foram propostos na literatura. Eles podem ser divididos em dois grupos: modelos do tipo freezing e modelos do tipo thawing [18]. Nos modelos freezing, o campo evoluía através do potencial no passado, mas a evolução gradualmente diminui depois do sistema entrar na fase de aceleração cósmica. Os potenciais desse tipo são, por exemplo,

$$
\begin{aligned}
& V(\phi)=M^{4+n} \phi^{-n}(n>0) \\
& V(\phi)=M^{4+n} \phi^{-n} \exp \left(\alpha \phi^{2} / m_{\mathrm{pl}}^{2}\right),
\end{aligned}
$$

onde $m_{\mathrm{pl}}=1 / \sqrt{G}$. O primeiro potencial não possui um mínimo, portanto, o campo evolui através do potencial em direção ao infinito. O segundo potencial possui um mínimo, no qual o campo eventualmente fica aprisionado (correspondendo a $\left.\omega_{\phi}=-1\right)$.

Nos modelos thawing, o campo escalar (com massa $m_{\phi}$ ) fica congelado devido à fricção causada pelo termo $H \dot{\phi}$ até recentemente, quando então ele começa a evoluir assim que $H$ torna-se menor que $m_{\phi}$. A equação de estado da energia escura é $\omega_{\phi} \simeq-1$ em tempos remotos, contudo ela começa a crescer em tempos tardios. Os potenciais representativos dessa classe são

$$
\begin{aligned}
& V(\phi)=V_{0}+M^{4-n} \phi^{n}(n>0) \\
& V(\phi)=M^{4} \cos ^{2}(\phi / f) .
\end{aligned}
$$

No caso do primeiro potencial, o universo colapsará no futuro se o sistema entrar na região com $V(\phi)<0$. No caso do segundo potencial, o campo escalar fica aproximadamente congelado no máximo do potencial durante o período no qual a massa do campo $m_{\phi}$ é menor que $H$, porém ele começa a evoluir ao redor do tempo presente $\left(m_{\phi} \simeq H_{0}\right)$. 


\subsubsection{Sistema Dinâmico}

Para podermos estudar a dinâmica cosmológica na presença de um campo escalar e de fluidos da matéria e assim verificar se a presença de um campo escalar é compatível com o que sabemos da evolução cósmica, é conveniente introduzir as variáveis adimensionais [18,35],

$$
x_{1} \equiv \frac{\kappa \dot{\phi}}{\sqrt{6} H}, \quad x_{2} \equiv \frac{\kappa \sqrt{V}}{\sqrt{3} H} .
$$

Assim, a equação (4.58) pode ser escrita como

$$
\Omega_{M} \equiv \frac{\kappa^{2} \rho_{M}}{3 H^{2}}=1-x_{1}^{2}-x_{2}^{2}
$$

A fração de energia escura é dada por

$$
\Omega_{\phi} \equiv \frac{\kappa^{2} \rho_{\phi}}{3 H^{2}}=x_{1}^{2}+x_{2}^{2} .
$$

Isso faz com que a relação $\Omega_{M}+\Omega_{\phi}=1$ seja satisfeita. Da equação (4.59), obtemos

$$
\frac{\dot{H}}{H^{2}}=-3 x_{1}^{2}-\frac{3}{2}\left(1+\omega_{M}\right)\left(1-x_{1}^{2}-x_{2}^{2}\right) .
$$

Nesse caso, a equação de estado efetiva (3.91) é dada por

$$
\omega_{\mathrm{ef}}=\omega_{M}+\left(1-\omega_{M}\right) x_{1}^{2}-\left(1+\omega_{M}\right) x_{2}^{2} .
$$

A equação de estado da energia escura (4.57), por sua vez, pode ser escrita como

$$
\omega_{\phi}=\frac{x_{1}^{2}-x_{2}^{2}}{x_{1}^{2}+x_{2}^{2}}
$$

Diferenciando as variáveis $x_{1}$ e $x_{2}$ com respeito ao número de $e$-foldings $N=\ln a$ juntamente com o uso das equações (4.60) e (4.68), obtemos as equações

$$
\begin{aligned}
& \frac{\mathrm{d} x_{1}}{\mathrm{~d} N}=-x_{1}+\frac{\sqrt{6}}{2} \lambda x_{2}^{2}+\frac{3}{2} x_{1}\left[\left(1-\omega_{M}\right) x_{1}^{2}+\left(1+\omega_{M}\right)\left(1-x_{2}^{2}\right)\right] \\
& \frac{\mathrm{d} x_{2}}{\mathrm{~d} N}=-\frac{\sqrt{6}}{2} \lambda x_{1} x_{2}+\frac{3}{2} x_{2}\left[\left(1-\omega_{M}\right) x_{1}^{2}+\left(1+\omega_{M}\right)\left(1-x_{2}^{2}\right)\right]
\end{aligned}
$$

onde

$$
\lambda \equiv-\frac{V_{, \phi}}{\kappa V}
$$


A quantidade $\lambda$ caracteriza a inclinação do potencial do campo escalar, e obedece a equação

$$
\frac{\mathrm{d} \lambda}{\mathrm{d} N}=-\sqrt{6} \lambda^{2}(\Gamma-1) x_{1}
$$

onde

$$
\Gamma \equiv \frac{V V_{, \phi \phi}}{V_{, \phi}^{2}}
$$

Se $\lambda$ for constante, a integração da equação (4.73) fornece o potencial exponencial

$$
V(\phi)=V_{0} e^{\kappa \lambda \phi}
$$

Da equação (4.75), esse potencial corresponde a $\Gamma=1$. Nesse caso, as equações autônomas (4.71) e (4.72) formam um sistema fechado. Para potenciais gerais, $\Gamma$ não é necessariamente uma constante. Nesses casos, precisamos obter o campo $\phi$ como uma função de $N$ ao resolvermos as equações (4.68) e (4.72) juntamente com a relação $\kappa \sqrt{V}=\sqrt{3} H x_{2}$. Dessa maneira, podemos determinar a evolução da variável $\lambda=\lambda(\phi)$.

\section{Potencial Exponencial}

Nesta seção, ilustraremos o formalismo da análise dinâmica das equações cosmológicas para modelos de quintessência, para isso nos deteremos no caso mais simples que é o caso de um potencial do tipo exponencial. Os pontos fixos do sistema podem ser encontrados ao considerarmos $\mathrm{d} x_{1} / \mathrm{d} N=\mathrm{d} x_{2} / \mathrm{d} N=0$ nas equações (4.71) e (4.72). Os pontos fixos são geralmente a solução do sistema dinâmico [16]. Quando $\lambda$ é constante, eles são dados por

(a) $\left(x_{1}, x_{2}\right)=(0,0), \quad \Omega_{\phi}=0, \omega_{\text {ef }}=\omega_{M}, \omega_{\phi}$ : indefinida.

(b1) $\left(x_{1}, x_{2}\right)=(1,0), \quad \Omega_{\phi}=1, \quad \omega_{\mathrm{ef}}=1, \quad \omega_{\phi}=1$.

(b2) $\left(x_{1}, x_{2}\right)=(-1,0), \quad \Omega_{\phi}=1, \quad \omega_{\text {ef }}=1, \quad \omega_{\phi}=1$.

(c) $\left(x_{1}, x_{2}\right)=\left(\lambda / \sqrt{6},\left[1-\lambda^{2} / 6\right]^{1 / 2}\right), \quad \Omega_{\phi}=1, \quad \omega_{\text {ef }}=-1+\lambda^{2} / 3$, $\omega_{\phi}=-1+\lambda^{2} / 3$.

(d) $\left(x_{1}, x_{2}\right)=\left(\sqrt{3 / 2}\left(1+\omega_{m}\right) / \lambda,\left[3\left(1-\omega_{M}^{2}\right) / 2 \lambda^{2}\right]^{1 / 2}\right), \quad \Omega_{\phi}=3\left(1+\omega_{M}\right) / \lambda^{2}$, $\omega_{\mathrm{ef}}=\omega_{M}, \omega_{\phi}=\omega_{M}$. 
O ponto (a) é uma solução dominada pelo fluido da matéria $\left(\Omega_{M}=1\right)$. A energia cinética da quintessência é dominante para os pontos (b1) e (b2), caso em que $\rho_{\phi}$ decresce rapidamente $\left(\rho_{\phi} \propto a^{-6}\right)$ relativamente a densidade de fundo. O ponto (c) corresponde a uma solução dominada pelo campo escalar e existe para $\lambda^{2}<6$. A aceleração cósmica ocorre se tivermos $\omega_{\text {ef }}<-1 / 3$, isto é, $\lambda^{2}<2$. O ponto (d) é a solução do tipo scaling para a qual a razão $\Omega_{\phi} / \Omega_{M}$ é uma constante diferente de zero. A existência da solução do tipo scaling exige que $\lambda^{2}>3\left(1+\omega_{M}\right)$ devido à exigência de que $\Omega_{\phi}<1$. Como $\omega_{\phi}=\omega_{M}$ para a solução scaling, não é possível termos aceleração cósmica a não ser que o fluido da matéria tenha uma equação de estado $\omega_{M}<-1 / 3$.

Para determinar se os pontos fixos $\left(x_{1}^{(c)}, x_{2}^{(c)}\right)$ determinados acima são estáveis, temos que considerar perturbações lineares $\left(\delta x_{1}, \delta x_{2}\right)$ ao redor dos pontos fixos [16, $36]$,

$$
x_{1}=x_{1}^{(c)}+\delta x_{1}, \quad x_{2}=x_{2}^{(c)}+\delta x_{2} .
$$

Linearizando as equações (4.71) e (4.72), obtemos o sistema de equações diferenciais

$$
\frac{\mathrm{d}}{\mathrm{d} N}\left(\begin{array}{l}
\delta x_{1} \\
\delta x_{2}
\end{array}\right)=\mathcal{M}\left(\begin{array}{l}
\delta x_{1} \\
\delta x_{2}
\end{array}\right),
$$

onde $\mathcal{M}$ é uma matriz $2 \times 2$ cujas componentes dependem de $x_{1}^{(c)}$ e $x_{2}^{(c)}$. A solução linearizada totalmente geral ao redor de cada ponto fixo pode ser escrita como

$$
x_{i}=x_{i}^{(c)}+\alpha_{i 1} e^{\mu_{1} N}+\alpha_{i 2} e^{\mu_{2} N}
$$

onde $\mu_{1}$ e $\mu_{2}$ são os autovalores da matriz $\mathcal{M}$ e $\alpha_{i 1}$ e $\alpha_{i 2}$ são coeficientes obtidos dos autovetores de $\mathcal{M}$.

Denominando o determinante da matriz $\mathcal{M}$ como $\mathcal{D}$, a estabilidade dos pontos fixos podem ser classificados como $[16,36]$

(i) Estável: $\mathcal{D}>0$ e $\mu_{1}<0, \mu_{2}<0$.

(ii) Instável: $\mathcal{D}>0$ e $\mu_{1}>0, \mu_{2}>0$.

(iii) Ponto de cela: $\mathcal{D}>0$ e $\mu_{1}>0, \mu_{2}<0$ (ou $\left.\mu_{1}<0, \mu_{2}>0\right)$.

(iv) Espiral estável: $\mathcal{D}<0$ e as partes reais de $\mu_{1}$ e $\mu_{2}$ negativas.

(v) Espiral instável: $\mathcal{D}<0$ e as partes reais de $\mu_{1}$ e $\mu_{2}$ positivas.

Se $\mathcal{D}=0$, a matriz $\mathcal{M}$ é singular e o sistema torna-se efetivamente unidimensional ao redor do ponto fixo. Essa classificação pode ser estendida para mais dimensões. Um 
ponto fixo será estável se as partes reais de seus autovalores forem negativas, instável se elas forem positivas e de cela quando temos tanto partes reais positivas quanto negativas. Se algum autovalor for zero, a estabilidade pode ser obtida expandindo a solução em ordens mais altas.

Os autovalores dos pontos fixos acimas são dados por $[16,18]$

(a) $\mu_{1}=-\frac{3}{2}\left(1-\omega_{M}\right), \mu_{2}=\frac{3}{2}\left(1+\omega_{M}\right)$.

(b1) $\mu_{1}=3-\frac{\sqrt{6}}{2} \lambda, \mu_{2}=3\left(1-\omega_{M}\right)$.

(b2) $\mu_{1}=3+\frac{\sqrt{6}}{2} \lambda, \mu_{2}=3\left(1-\omega_{M}\right)$.

(c) $\mu_{1}=\frac{1}{2}\left(\lambda^{2}-6\right), \mu_{2}=\lambda^{2}-3\left(1+\omega_{M}\right)$.

(d) $\mu_{1,2}=-\frac{3\left(1-\omega_{M}\right)}{4}\left[1 \pm \sqrt{1-\frac{8\left(1+\omega_{M}\right)\left[\lambda^{2}-3\left(1+\omega_{M}\right)\right]}{\lambda^{2}\left(1-\omega_{M}\right)}}\right]$.

Considerando somente o caso em que a equação de estado da matéria está na região $0 \leq \omega_{M} \leq 1$, a estabilidade dos pontos fixos pode ser resumida como

(a) Ponto de cela.

(b1) Instável para $\lambda<\sqrt{6}$ e do tipo cela para $\lambda>\sqrt{6}$.

(b2) Instável para $\lambda>-\sqrt{6}$ e do tipo cela para $\lambda<-\sqrt{6}$.

(c) Estável para $\lambda^{2}<3\left(1+\omega_{M}\right)$ e do tipo cela para $3\left(1+\omega_{M}\right)<\lambda^{2}<6$.

(d) Do tipo cela para $\lambda^{2}<3\left(1+\omega_{M}\right)$, estável para $3\left(1+\omega_{M}\right)<\lambda^{2}<\frac{24\left(1+\omega_{M}\right)^{2}}{7+9 \omega_{M}}$ e espiral estável para $\lambda^{2}>\frac{24\left(1+\omega_{M}\right)^{2}}{7+9 \omega_{M}}$.

As épocas dominadas pela radiação $\left(\omega_{M}=1 / 3\right)$ e pela matéria $\left(\omega_{M}=0\right)$ podem ser realizadas tanto pelo ponto (a) quanto pelo (d). Quando $\lambda^{2}>3\left(1+\omega_{M}\right)$ as soluções aproximam-se do ponto estável (d) ao invés do ponto (a). Nesse caso, porém, a solução não deixa a era scaling $\left(\Omega_{\phi}=\right.$ constante) para alcançar a época da aceleração. Para permitir que $\Omega_{\phi}$ evolua para alcançar $\Omega_{M}$, precisamos que a inclinação do potencial gradualmente diminua. Isso pode ser realizado por potenciais em que $\lambda$ fique menor com o tempo (como o $\left.V(\phi)=M^{4+n} \phi^{-n}\right)$.

O ponto (c) é o único ponto fixo que permite um atrator estável e que leva à aceleração do universo para $\lambda^{2}<2$. Quando $\lambda^{2}<2$, uma solução (d) fisicamente viável não existe por causa que $\Omega_{\phi}>1$ tanto para um fluido de radiação quanto para um de matéria escura. Nesse caso, as épocas dominadas pela radiação e pela matéria são dadas pelo ponto (a). Podemos notar que, quando $\lambda$ é próximo de 
zero, a solução que começa no ponto (a) e aproxima-se do ponto (c) não é muito diferente de uma constante cosmológica. Contudo, ainda assim podemos encontrar uma diferença do caso que $\omega_{\phi}=-1$, visto que a equação de estado para o atrator é dada por $\omega_{\phi}=-1+\lambda^{2} / 3$.

Com essa análise, vemos que os modelos de quintessência são cosmologicamente viáveis. Além disso, eles possuem características distintas daquelas da constante cosmológica, como, por exemplo, a equação de estado atual da energia escura e quão longo foi o período dominado pela matéria. Essas previsões diferentes poderão, no futuro, ser comparadas com as observações cosmológicas. 


\section{Capítulo 5}

\section{Interação no Setor Escuro na Linguagem de Fluidos Interagentes}

Como vimos anteriormente, no modelo cosmológico padrão, a energia e a matéria escura são os componentes dominantes da evolução tardia do universo. Nesse caso, a energia e a matéria escura evoluem independentemente uma da outra. Como uma possível extensão desse cenário, podemos considerar uma interação no setor escuro do universo. Uma motivação para isso é que esse acoplamento entre a energia e a matéria escura poderia ajudar a explicar o porquê da energia escura somente se tornar dominante depois da formação das galáxias e de outras estruturas e assim aliviar o problema da coincidência. Este capítulo tratará dessa interação em um formalismo em que todos os componentes do universo são descritos por fluidos perfeitos e o termo de interação é dado por um ansatz baseado nas grandezas envolvidas na equação de balanço de um fluido.

\subsection{Universo Homogêneo e Isotrópico}

Um acoplamento geral no setor escuro pode ser descrito, no fundo cosmológico homogêneo e isotrópico, através das equações de conservação da matéria escura $(c)$ e da energia escura $(d)[1-3]$,

$$
\begin{aligned}
& \rho_{c}^{\prime}=-3 \mathcal{H} \rho_{c}+a Q \\
& \rho_{d}^{\prime}=-3 \mathcal{H}(1+\omega) \rho_{d}-a Q,
\end{aligned}
$$

onde $\omega=P_{d} / \rho_{d}, \mathcal{H}=\mathrm{d} \ln a / \mathrm{d} \eta$ e $\eta$ é o tempo conforme (lembrando que a métrica de FRW plana em função do tempo conforme pode ser escrita como $\mathrm{d} s^{2}=-a^{2} \mathrm{~d} \eta^{2}+$ 
$\left.a^{2} \mathrm{~d} \mathbf{x}^{2}\right)$. Nas equações de balanço, $Q$ é a taxa de transferência de energia; de modo que $Q<0(>0)$ implica que a direção de transferência de energia é da matéria escura para a energia escura (energia escura para a matéria escura).

Nessas equações, qualquer que seja a forma fenomenológica da interação entre os componentes do setor escuro, $Q$ deve ser, devido às grandezas envolvidas no lado esquerdo das equações de balanço, uma função das densidades de energia multiplicadas por uma quantidade com unidade do inverso do tempo. Em um contexto cosmológico, a escolha mais óbvia para essa quantidade com unidade do inverso do tempo é o fator de Hubble, $H$ [1]. Em primeira ordem nas densidades de energia tanto da matéria escura quanto da energia escura, $Q$ pode ser expresso como uma função ou de cada uma dessas densidades em separado, ou da soma das duas densidades.

Aqui, consideraremos $Q$ como uma combinação linear das densidades de energia do setor escuro, isto é, $Q=3 H\left(\lambda_{1} \rho_{c}+\lambda_{2} \rho_{d}\right)$. No estudo da estabilidade do modelo de interação fenomenológico, veremos que, quando a interação é proporcional à densidade de energia escura, $Q=3 H \lambda_{2} \rho_{d}$, temos uma perturbação da curvatura estável, isto é, sem a presença de nenhum mau comportamento. Contudo, quando a interação é proporcional à densidade de energia da matéria escura, $Q=3 H \lambda_{1} \rho_{c}$, ou do setor escuro total, $Q=3 H \lambda\left(\rho_{c}+\rho_{d}\right)$, a perturbação da curvatura só pode ser estável quando a equação de estado da energia escura, considerada constante, satisfaz $\omega<-1$

Quando um acoplamento no setor escuro é considerado, a evolução das densidades do setor escuro é distinta da evolução do modelo padrão. Podemos utilizar como parâmetro dessa mudança uma equação de estado efetiva para o setor escuro, de forma que podemos descrever o modelo acoplado como equivalente a um modelo não-acoplado no fundo cosmológico. Escrevendo $\rho_{c}^{\prime}+3 \mathcal{H}\left(1+\omega_{c, \text { ef }}\right) \rho_{c}=0$ e $\rho_{d}^{\prime}+3 \mathcal{H}\left(1+\omega_{d, \mathrm{ef}}\right) \rho_{d}=0$, temos que [4]

$$
\omega_{c, \text { ef }}=-\frac{a Q}{3 \mathcal{H} \rho_{c}}, \quad \omega_{d, \text { ef }}=\omega+\frac{a Q}{3 \mathcal{H} \rho_{d}} .
$$

Quando $Q<0$, temos que $\omega_{c \text {,ef }}>0$, de forma que a matéria escura evolui mais rapidamente do que $a^{-3}$, enquanto $\omega_{d, \text { ef }}<\omega$, de forma que a energia escura possui mais poder de aceleração. O oposto ocorre para $Q>0$. Quando $Q<0$, a energia escura acoplada pode se comportar como um modelo phantom desacoplado, isto é, com $\omega_{d, \text { ef }}<-1$, mas sem os problemas geralmente associados com a energia escura phantom [37]. Escolhendo diferentes formas para a interação, as equações de estado efetivas comportam-se diferentemente, como pode ser visto na Tabela 5.1.

Definimos $r=\rho_{c} / \rho_{d}$ como a razão das densidades de energia da matéria e da 
Tabela 5.1: Modelos de Interação

\begin{tabular}{|c|c|c|c|c|c|}
\hline Modelo & $Q$ & $\omega$ & $\omega_{c, \text { ef }}$ & $\omega_{d, \text { ef }}$ & Vínculos \\
\hline I & $3 H \lambda_{2} \rho_{d}$ & $-1<\omega<0$ & $-\lambda_{2} / r$ & $\omega+\lambda_{2}$ & $\lambda_{2}<-2 \omega \Omega_{c}$ \\
\hline II & $3 H \lambda_{2} \rho_{d}$ & $\omega<-1$ & $-\lambda_{2} / r$ & $\omega+\lambda_{2}$ & $\lambda_{2}<-2 \omega \Omega_{c}$ \\
\hline III & $3 H \lambda_{1} \rho_{c}$ & $\omega<-1$ & $-\lambda_{1}$ & $\omega+\lambda_{1} r$ & $0<\lambda_{1}<-\omega / 4$ \\
\hline IV & $3 H \lambda\left(\rho_{c}+\rho_{d}\right)$ & $\omega<-1$ & $-\lambda(1+1 / r)$ & $\omega+\lambda(r+1)$ & $0<\lambda<-\omega / 4$ \\
\hline
\end{tabular}

energia escura. Com o intuito de aliviar o problema da coincidência, requereremos que a razão $r$ seja uma constante durante parte da história da expansão do universo, de forma que a atual observação de que as densidades de energia do setor escuro possuem a mesma ordem de grandeza não seja uma coincidência forte. O regime em que $r$ é uma constante é conhecido como regime de scaling [38]. No caso em que temos $r$ constante e $r>1, \Omega_{d}$ é, no passado, diferente de zero por um tempo considerável e assim o problema da coincidência é aliviado. Já no caso em que temos $r$ constante e $r<1$, o comportamento de scaling fica no presente ou no futuro, de forma que o início da época de aceleração do universo, onde $r \simeq 1$, não é mais um ponto especial da história do universo, visto que um regime de scaling logo será alcançado. Esse caso também alivia o problema da coincidência. A suposição da existência de um regime de scaling nos leva à condição $r^{\prime}=\frac{\rho_{c}^{\prime}}{\rho_{d}}-r \frac{\rho_{d}^{\prime}}{\rho_{d}}=0$, que nos dá a equação quadrática

$$
\lambda_{1} r^{2}+\left(\lambda_{1}+\lambda_{2}+\omega\right) r+\lambda_{2}=0 .
$$

Quando o acoplamento é proporcional à densidade da energia escura (modelos I e II), isto é, $\lambda_{1}=0$, a equação (5.4) possui somente uma raiz, $r=-\frac{\lambda_{2}}{\lambda_{2}+\omega}$. Foi mostrado em [1] que quando o acoplamento é proporcional à densidade de energia escura, $\lambda_{2}>-2 \omega \Omega_{c}$ leva a uma densidade de energia da matéria escura negativa no passado, o que é uma situação não-física. Assim, exigimos que $\lambda_{2}<-2 \omega \Omega_{c}$, o que mostra que nossa raiz para $r$ está no futuro, isto é, $r<1$. Já a equação de estado efetiva da energia escura torna-se $\omega_{d, \mathrm{ef}}=\omega+\lambda_{2}$. Isso mostra que o acoplamento e a equação de estado da energia escura estão emaranhados. Assim, é impossível distinguir uma da outra ao investigarmos somente a evolução de fundo.

Quando o acoplamento é proporcional à densidade de matéria escura ou à densidade de todo o setor escuro (modelos III, IV), temos duas raízes para a equação quadrática (5.4) 


$$
\begin{aligned}
& \left(r \lambda_{1}\right)_{1}=-\frac{1}{2}\left(\omega+\lambda_{1}+\lambda_{2}\right)+\frac{1}{2} \sqrt{\left(\lambda_{1}+\lambda_{2}+\omega\right)^{2}-4 \lambda_{1} \lambda_{2}} \\
& \left(r \lambda_{1}\right)_{2}=-\frac{1}{2}\left(\omega+\lambda_{1}+\lambda_{2}\right)-\frac{1}{2} \sqrt{\left(\lambda_{1}+\lambda_{2}+\omega\right)^{2}-4 \lambda_{1} \lambda_{2}} .
\end{aligned}
$$

A primeira raiz aparece no passado e a segunda aparece no futuro. Para a interação proporcional à densidade de matéria escura (modelo III) $\left(\lambda_{2}=0\right.$ ), quando $\lambda_{1}<$ $-\omega / 4$, as raízes são reais e podem ser simplificadas, de forma que temos que

$$
\begin{aligned}
& \left(r \lambda_{1}\right)_{1}=-\left(\omega+\lambda_{1}\right)>0 \\
& \left(r \lambda_{1}\right)_{2}=0 .
\end{aligned}
$$

A equação de estado, nesse caso, se comporta diferentemente no passado e no futuro. No universo jovem, a equação de estado efetiva é da forma

$$
\omega_{d, \mathrm{ef}} \approx-\lambda_{1}
$$

Quando o universo evolui para o tempo presente ou futuro, temos que

$$
\omega_{d, \mathrm{ef}} \approx \omega
$$

Essa expressão não depende do acoplamento, eliminando a degenerescência entre a equação de estado da energia escura e o acoplamento.

Quando a interação é proporcional à densidade de energia total dos componentes do setor escuro (modelo IV com $\lambda_{1}=\lambda_{2}=\lambda$ ), a equação (5.4) tem duas raízes reais quando $\lambda<-\omega / 4$

$$
\begin{aligned}
(r \lambda)_{1} & =\frac{1}{2}(\omega+2 \lambda)+\frac{1}{2} \sqrt{(2 \lambda+\omega)^{2}-4 \lambda^{2}} \\
& \approx-(\omega+2 \lambda) \\
(r \lambda)_{2} & =\frac{1}{2}(\omega+2 \lambda)-\frac{1}{2} \sqrt{(2 \lambda+\omega)^{2}-4 \lambda^{2}} \\
& \approx-\frac{\lambda^{2}}{\omega} \approx \lambda^{2} .
\end{aligned}
$$

Isso nos leva a uma equação de estado para a energia escura da forma $\omega_{d, \mathrm{ef}} \approx-\lambda$ no passado e da forma $\omega_{d, \mathrm{ef}} \approx \omega+\lambda+\lambda^{2}$ no futuro.

Em geral, essa degenerescência não pode ser quebrada somente analisando a 
dinâmica do universo homogêneo e isotrópico, assim, para entendermos melhor as consequências de nosso modelo, devemos abandonar o universo homogêneo e isotrópico e estudar a evolução de um universo inomogêneo com pequenas perturbações lineares. Entretanto, antes disso, podemos fazer uma análise termodinâmica da interação entre os componentes do setor escuro no universo homogêneo e isotrópico para tentar antever a direção da transferência de energia no setor escuro.

\subsubsection{Considerações Termodinâmicas sobre a Interação no Setor Escuro}

Consideraremos, nesta seção, o universo tardio como sendo composto por dois fluidos representando o setor escuro, desprezando, assim, a contribuição da radiação, o que é uma ótima aproximação. Como a energia escura e a matéria escura são representados por fluidos clássicos, elas respeitam as leis da termodinâmica clássica. Obviamente, isso deixa de ser válido se a energia escura for um campo escalar em um estado puramente quântico, visto que, nessa situação, sua entropia se anula e não podemos definir uma temperatura. Mas, mesmo utilizando campos escalares para representar a energia escura, essa situação não é o caso mais geral. Podemos, por exemplo, considerar a energia escura como uma manifestação efetiva de uma mistura de campos escalares. Geralmente, essa mistura não estará em um estado puramente quântico o que nos permite definir uma entropia e uma temperatura global.

Se esses dois componentes conservam-se separadamente, temos, em um universo de FRW, que

$$
\begin{aligned}
& \dot{\rho}_{c}+3 H\left(\rho_{c}+P_{c}\right)=0 \\
& \dot{\rho}_{d}+3 H(1+\omega) \rho_{d}=0,
\end{aligned}
$$

onde temos a derivada temporal sendo representada por um ponto. A equação de estado da matéria escura pode ser aproximadamente escrita em forma paramétrica como $[39,40]$

$$
\begin{aligned}
& \rho_{c}=n_{c} M+\frac{3}{2} n_{c} T_{c} \\
& P_{c}=n_{c} T_{c}
\end{aligned}
$$

onde, lembrando, a constante de Boltzmann, nas unidades utilizadas, é dada pela unidade, $k_{B}=1$, e $T_{c} \ll M$, com $M$ sendo a massa das partículas da matéria 
escura. Como consequência dessa parametrização, temos que $\rho_{c} \sim a^{-3}$. Para a energia escura, temos da equação (5.10) que $\rho_{d} \propto \exp \left[\int-3(1+\omega) \mathrm{d} a / a\right]$.

Utilizando a equação da primeira lei da termodinâmica, $T \mathrm{~d}(s V)=\mathrm{d}(\rho V)+P \mathrm{~d} V$, e o fato que $s=\frac{\rho+P}{T}$ ( $s$ é a densidade de entropia), temos que a evolução da temperatura é dada por

$$
\frac{\dot{T}}{T}=-3 H\left(\frac{\partial P}{\partial \rho}\right)_{V} .
$$

Utilizando essa equação, a dependência das temperaturas com o fator de escala é dada por [40]

$$
\begin{aligned}
& T_{c} \propto a^{-2} \\
& T_{d} \propto \exp \left(-\int \mathrm{d} a \frac{3 \omega}{a}\right) .
\end{aligned}
$$

As equações (5.13) sugerem que atualmente temos $T_{c} \ll T_{d}$ e que o contrário era verdade no universo jovem (com $T_{c} \ll M$ ainda sendo respeitado).

Quando os dois componentes interagem, as equações (5.10) generalizam para as expressões

$$
\begin{aligned}
& \dot{\rho}_{c}+3 H\left(\rho_{c}+P_{c}\right)=Q \\
& \dot{\rho}_{d}+3 H(1+\omega) \rho_{d}=-Q .
\end{aligned}
$$

Aqui, por ora, assumiremos que o termo de interação possui a forma

$$
Q=3 H \lambda \rho_{d}
$$

onde $\lambda$ é uma pequena constante. Temos, assim, que $\dot{\rho}_{d}+3 H(1+\omega+\lambda) \rho_{d}=0$. Utilizando a relação $\frac{\dot{T}_{d}}{T_{d}}=-3(\omega+\lambda) \frac{\dot{a}}{a}$, obtemos

$$
T_{d} \propto \exp \left(\int-3(\omega+\lambda) \mathrm{d} a / a\right) .
$$

Consequentemente, se $\lambda>0$, isto é, $Q>0, T_{d}$ crescerá mais lentamente conforme o universo expande-se do que na ausência da interação. Por sua vez, $T_{c}$ decrescerá mais lentamente. Isso é consistente com a segunda lei da termodinâmica que implica que quando dois sistemas não estão em equilíbrio mútuo (térmico ou qualquer outro tipo de equilíbrio) e interagem entre si, a interação tende a levar os sistemas para um 
equilíbrio comum. Se deixados interagindo entre si, eles eventualmente atingirão o equilíbrio. No caso aqui exposto, o equilíbrio nunca é alcançado por causa da expansão do universo (que pode ser vista como um agente externo) que atua no sentido oposto. Se $\lambda$ for negativo, temos que $Q<0$, e a diferença de temperatura aumentará cada vez mais, algo contrário a segunda lei da termodinâmica. Claramente, nossa conclusão depende da validade de (5.15).

Considerando as relações (5.14), vemos que $Q$ deve ser uma função das densidades de energia multiplicadas por uma quantidade com unidades do inverso do tempo. Uma escolha óbvia é a constante de Hubble $H$; assim $Q=f\left(H \rho_{c}, H \rho_{d}\right)$, com $f$ uma função analítica qualquer e, como feito anteriormente, ao expandirmos em primeira ordem essa função, temos $Q \simeq \lambda_{1} H \rho_{c}+\lambda_{2} H \rho_{d}$. No regime em que $\rho_{c} / \rho_{d}$ é uma constante, a equação (5.15) é facilmente recuperada. Fora desse regime particular, excluindo tempos muito remotos, espera-se que essa razão varie lentamente (isto é, não muito mais rápido que o fator de escala $a(t)$ ), de forma que podemos considerála constante por partes durante a evolução do universo. Assim, recuperamos mais uma vez a equação (5.15), agora, contudo, $\lambda$ é constante por partes.

Podemos entender melhor esse cenário ao analisarmos o princípio de Le ChâtelierBraun [40,41], que diz que quando um sistema é perturbado para fora de seu estado de equilíbrio ele reage tentando recuperar o velho estado de equilíbrio ou atingir um novo. Em tempos suficientemente remotos, temos que $T_{c}>T_{d}$, e a expansão do universo rapidamente leva ambos os sistemas para uma temperatura de equilíbrio comum $T_{\text {eq }}$ em $a=a_{\text {eq }}$. Contudo, logo temos que $a>a_{\text {eq }}$ e o equilíbrio térmico é perdido, $T_{c}<T_{\text {eq }}<T_{d}$. No nosso caso, a resposta do sistema para a perda do equilíbrio é uma transferência continua de energia para a matéria escura. Mesmo que isso não leve o sistema para nenhum novo estado de equilíbrio, temos um cenário no qual a taxa em que o sistema move-se para além do equilíbrio é menor.

\subsection{Formalismo Perturbativo}

No universo homogêneo, a equação de balanço do fluido $A$ é dada por

$$
\rho_{A}^{\prime}=-\mathcal{H}\left(1+\omega_{A}\right) \rho_{A}+a Q_{A},
$$

onde $\mathcal{H}=a^{\prime} / a$ e a linha representa derivada em relação ao tempo conforme.

No universo perturbado, existem algumas peculiaridades e complicações que não existem na dinâmica do fundo homogêneo e isotrópico. Primeiro, precisamos de uma forma covariante para a transferência de energia e de momento entre os componentes do setor escuro que seja verdadeira em um universo inomogêneo e que se reduza à 
forma de fundo em um universo de FRW. Por exemplo, se utilizarmos o ansatz $Q=Q_{0} a^{n}$, então a dinâmica de fundo pode ser determinada e os parâmetros $Q_{0}$ e $n$ podem ser limitados por observações geométricas. Contudo, não existe uma forma covariante para tais ansatzes, de forma que não podemos calcular as perturbações.

Segundo, precisamos garantir que as perturbações da energia escura sejam estáveis, isto é, $c_{s, d}^{2}>0$, onde $c_{s, d}$ é a velocidade do som da energia escura (velocidade com que as flutuações de pressão se propagam). Para um modelo de campo escalar para a energia escura, temos $c_{s, d}^{2}$, sem que nenhuma suposição extra seja necessária. Entretanto, para modelos de fluidos, devemos exigir que $c_{s, d}^{2}>0$ de forma arbitrária, de modo que o fluido da energia escura é efetivamente não-adiabático.

Com tais ressalvas em mente, exploraremos a teoria de perturbação em primeira ordem na presença de um acoplamento entre energia e matéria escura. $\mathrm{O}$ espaço-tempo perturbado em primeira ordem, considerando somente as perturbações escalares, é dado por [4]

$$
\mathrm{d} s^{2}=a^{2}\left[-(1+2 \psi) \mathrm{d} \eta^{2}+2 \partial_{i} B \mathrm{~d} \eta \mathrm{d} x^{i}+(1+2 \phi) \delta_{i j} \mathrm{~d} x^{i} \mathrm{~d} x^{j}+D_{i j} E \mathrm{~d} x^{i} \mathrm{~d} x^{j}\right],
$$

onde $\psi, B, \phi$ e $E$, novamente, representam as perturbações escalares da métrica e

$$
D_{i j}=\left(\partial_{i} \partial_{j}-\frac{1}{3} \delta_{i j} \nabla^{2}\right) .
$$

A quadrivelocidade, no universo de fundo, é $\bar{u}^{\mu}=a^{-1} \delta_{0}^{\mu}$, de forma que a quadrivelocidade do fluido perfeito $A$, em um universo perturbado linearmente, é

$$
u_{A}^{\mu}=a^{-1}\left(1-\psi, \partial^{i} v_{A}\right), \quad u_{A \mu}=a\left(-1-\psi, \partial_{i}\left[v_{A}+B\right]\right),
$$

onde $v_{A}$ é o potencial da velocidade peculiar do fluido [2]. A taxa de expansão do volume, que generaliza a relação newtoniana $\theta=\nabla \cdot \vec{v}$, é dada no espaço de Fourier por

$$
\theta_{A}=-k^{2}\left(v_{A}+B\right)
$$

Escolheremos $u_{A}^{\mu}$ como a quadrivelocidade no referencial da energia, isto é, queremos que o fluxo de momento seja igual a zero relativo a $u_{A}^{\mu}$, de forma que $T_{A \nu}^{\mu} u_{A}^{\nu}=-\rho_{A} u_{A}^{\mu}$. Assim, o tensor de energia-momento do fluido perfeito A pode ser escrito como [42]

$$
T_{A}^{\mu \nu}=\rho_{A} u_{A}^{\mu} u_{A}^{\nu}+P_{A}\left(g^{\mu \nu}+u_{A}^{\mu} u_{A}^{\nu}\right)
$$


onde $\rho_{A}=\bar{\rho}_{A}+\delta \rho_{A}$ e $P_{A}=\bar{P}_{A}+\delta P_{A}$. O tensor de energia-momento total, que é conservado, é $T^{\mu \nu}=\sum_{A} T_{A}^{\mu \nu}$, de forma que

$$
(\rho+P) u^{\mu} u^{\nu}+P g^{\mu \nu}+q^{\mu} u^{\nu}+q^{\nu} u^{\mu}=\sum_{A}\left[\left(\rho_{A}+P_{A}\right) u_{A}^{\mu} u_{A}^{\nu}+P_{A}\right]
$$

onde $q^{\mu}$ é o fluxo de momento total relativo a quadrivelocidade total $u^{\mu}$. Estamos considerando que os tensores de estresse anisotrópicos em nossos tensores de energiamomento são insignificantes, o que é uma boa aproximação para a matéria e energia escura. Em geral, a quadrivelocidade total $u^{\mu}$ possui a forma

$$
u^{\mu}=a^{-1}\left(1-\psi, \partial^{i} v\right) .
$$

A escolha de $v$ depende de como a quadrivelocidade total é escolhida.

Segue das equações (5.23) e (5.24) que $\rho=\sum \rho_{A}, P=\sum P_{A}$ e que o fluxo de momento total é $q^{i}=a^{-1} \sum_{A}\left(\rho_{A}+P_{A}\right) \partial^{i} v_{A}-a^{-1}(\rho+P) \partial^{i} v$. Assim, o referencial de energia total $\left(q^{i}=0\right)$ é definido por

$$
(\rho+P) v=\sum\left(\rho_{A}+P_{A}\right) v_{A}
$$

Essa será a escolha de $v$ que utilizaremos a partir de agora.

A forma covariante da transferência de energia-momento entre os fluidos que compõe o universo é

$$
\begin{aligned}
& \nabla_{\nu} T_{A}^{\mu \nu}=Q_{A}^{\mu}, \\
& \sum_{A} Q_{A}^{\mu}=0 .
\end{aligned}
$$

Uma transferência geral de energia-momento pode ser sempre dividida em relação à quadrivelocidade total como [42]

$$
\begin{aligned}
& Q_{A}^{\mu}=Q_{A} u^{\mu}+F_{A}^{\mu}, \\
& Q_{A}=\bar{Q}_{A}+\delta Q_{A}, \\
& u_{\mu} F_{A}^{\mu}=0,
\end{aligned}
$$

onde $\bar{Q}_{A}$ é a taxa de transferência da densidade de energia no fundo cosmológico, $\delta Q_{A}$ é $\bar{Q}_{A}$ perturbado em primeira ordem e $F_{A}^{\mu}=a^{-1}\left(0, \partial^{i} f_{A}\right)$, onde $f_{A}$ é o potencial 
de transferência intrínseca de momento. Temos, então, que

$$
\begin{aligned}
Q_{A}^{0} & =a^{-1}\left[Q_{A}(1-\psi)+\delta Q_{A}\right], \\
Q_{A}^{i} & =a^{-1} \partial^{i}\left[f_{A}+Q_{A} v\right] .
\end{aligned}
$$

Nessas equações, a barra em $\bar{Q}_{A}$ foi omitida para simplificar a notação, de forma que $Q_{A}$ representa a forma funcional da interação no fundo homogêneo e isotrópico. A transferência perturbada de energia inclui um termo dependente da métrica perturbada $Q_{A} \psi$ e um da perturbação intrínseca $\delta Q_{A}$. A transferência perturbada de momento é composta de duas partes: o potencial de transferência de momento $Q_{A} v$ que tem origem no transporte de energia através da velocidade total e um potencial de transferência intrínseca de momento $f_{A}$. No universo de fundo, o quadrivetor de transferência de energia-momento possui a forma

$$
Q_{c}^{\mu}=a^{-1}\left(Q_{c}, \overrightarrow{0}\right)=a^{-1}(Q, \overrightarrow{0})=-Q_{d}^{\mu}
$$

de forma que não temos transferência de momento.

A conservação de energia-momento implica em

$$
0=\sum Q_{A}=\sum \delta Q_{A}=\sum f_{A}
$$

Para cada fluido $A$, as equações (5.26) nos dão as equações perturbadas de balanço de energia e momento [2],

$$
\begin{gathered}
\delta \nabla_{\mu} T_{A}^{\mu 0}=\frac{1}{a^{2}}\left[-2\left(\rho_{A}^{\prime}+3 \mathcal{H}\left(P_{A}+\rho_{A}\right)\right) \psi+\delta \rho_{A}^{\prime}+\left(P_{A}+\rho_{A}\right) \theta_{A}\right. \\
\left.+3 \mathcal{H}\left(\delta P_{A}+\delta \rho_{A}\right)+3\left(P_{A}+\rho_{A}\right) \phi^{\prime}\right]=\delta Q_{A}^{0}, \\
\partial_{i} \delta \nabla_{\mu} T_{A}^{\mu i}=\frac{1}{a^{2}}\left[\left(P_{A}^{\prime}+\mathcal{H}\left(P_{A}+\rho_{A}\right)\right) \nabla^{2} B+\left(\left(P_{A}^{\prime}+\rho_{A}^{\prime}\right)+4 \mathcal{H}\left(P_{A}+\rho_{A}\right)\right) \theta_{A}\right. \\
\left.+\left(P_{A}+\rho_{A}\right) \nabla^{2} B^{\prime}+\nabla^{2} \delta P_{A}+\left(P_{A}+\rho_{A}\right) \theta_{A}^{\prime}+\left(P_{A}+\rho_{A}\right) \nabla^{2} \psi\right]=\partial_{i} \delta Q_{A}^{i} .
\end{gathered}
$$

Nessas equações, os termos $\delta Q_{A}^{0}$ e $\delta Q_{A}^{i}$ representam os termos de primeira ordem de (5.28) e (5.29), respectivamente. A partir de agora, desprezaremos a transferência de momento escolhendo $\delta Q_{A}^{i}=0$ (um potencial $f_{A}$ que permita essa escolha pode ser sempre considerado). Isso é uma escolha específica para a interação e os resultados não devem depender muito dela. Como nosso objetivo será estudar a estabilidade 
das equações perturbativas, essa escolha facilitará nossa análise e não alterará nossas conclusões finais.

Para relacionar a perturbação da pressão $\delta P$ com a perturbação da densidade de energia $\delta \rho$, precisamos introduzir a velocidade do som de um fluido interagente. A velocidade do som $c_{s A}$ de um fluido, ou de um campo escalar, é a velocidade de propagação das flutuações da pressão no referencial de repouso de $A$,

$$
c_{s A}^{2}=\left.\frac{\delta P_{A}}{\delta \rho_{A}}\right|_{r} .
$$

Para um campo escalar $\phi$, por exemplo, o referencial de repouso é definido pela hipersuperfície $\phi=$ constante, ortogonal a quadrivelocidade do referencial de repouso, $u_{\mu}^{\phi} \propto \nabla_{\mu} \phi$. Assim, a densidade de energia cinética no referencial de repouso é dada por $-\frac{1}{2} \nabla_{\mu} \phi \nabla^{\mu} \phi=\phi^{\prime 2} /\left(2 a^{2}\right)$. Além disso, visto que $\delta \phi=0$, no referencial de repouso, temos que $\delta V=0$, onde $V(\phi)$ é o potencial. Consequentemente, as perturbações de densidade e de pressão são iguais no referencial de repouso: $\delta \rho_{\phi}=\delta\left(\frac{1}{2} a^{-2} \phi^{\prime 2}+V\right)=a^{-2} \phi^{\prime} \delta \phi=\delta\left(\frac{1}{2} a^{-2} \phi^{\prime 2}-V\right)=\delta P_{\phi}$. A velocidade do som é, portanto, igual à velocidade da luz, independentemente da forma de $V(\phi)$,

$$
\left.\delta \phi\right|_{r}=0 \Rightarrow c_{s \phi}^{2}=1
$$

Podemos definir a velocidade adiabática do som para qualquer meio material através de

$$
c_{a A}^{2}=\frac{P_{A}^{\prime}}{\rho_{A}^{\prime}}=\omega_{A}+\frac{\omega_{A}^{\prime}}{\rho_{A}^{\prime} / \rho_{A}} .
$$

Para um fluido barotrópico, $c_{s}^{2}=c_{a}^{2}$, e se $\omega_{A}=$ constante, então $c_{a}^{2}=\omega_{A}$.

Trataremos a matéria escura como um fluido adiabático com velocidade do som igual a zero. O modelo de fluido para a energia escura com $\omega$ constante também é um modelo barotrópico adiabático. Todavia, se tratarmos a energia escura como um fluido estritamente adiabático, então a velocidade do som terá um valor imaginário $\left(c_{s d}^{2}=c_{a d}^{2}=\omega<0\right)$, levando à instabilidades na energia escura. Para evitar esse problema, é necessário impor $c_{s d}^{2}>0$, sendo natural adotar o valor do campo escalar (5.35) [2]. Assim,

$$
\begin{aligned}
& c_{s d}^{2}=1 \\
& c_{a d}^{2}=\omega=\text { const }<0 .
\end{aligned}
$$

Essa é a escolha feita, por exemplo, no código CAMB que é útil para resolver 
numericamente as equações cosmológicas perturbadas [43].

Nas equações perturbadas (5.32) e (5.33), precisamos relacionar $\delta P_{A} \operatorname{com} \delta \rho_{A}$ através de (5.34). O referencial de repouso de A, conhecido como gauge do momento zero ou gauge comóvel ortogonal, é o referencial comóvel $\left(\left.v_{A}\right|_{r}=0\right)$ e ortogonal $\left(\left.B\right|_{r}=0\right)$, de modo que nesse referencial temos que

$$
\left.T_{A 0}^{i}\right|_{r}=0=\left.T_{A i}^{0}\right|_{r}
$$

Fazendo a transformação de gauge, $x^{\mu}+\left(\delta \eta_{A}, \partial^{i} \delta x_{A}\right)$, do gauge do referencial de repouso para um gauge geral, obtemos que $[2,10]$

$$
\begin{aligned}
& v_{A}+B=\left.\left(v_{A}+B\right)\right|_{r}+\delta \eta_{A} \\
& \delta P_{A}=\left.\delta P_{A}\right|_{r}-P_{A}^{\prime} \delta \eta_{A} \\
& \delta \rho_{A}=\left.\delta \rho_{A}\right|_{r}-\rho_{A}^{\prime} \delta \eta_{A} .
\end{aligned}
$$

Assim, $\delta \eta_{A}=v_{A}+B$, e substituindo nas flutuações de pressão e de densidade, obtemos

$$
\delta P_{A}=c_{a A}^{2} \delta \rho_{A}+\left(c_{s A}^{2}-c_{a A}^{2}\right)\left[\delta \rho_{A}+\rho_{A}^{\prime}\left(v_{A}+B\right)\right]=c_{a A}^{2} \delta \rho_{A}+\delta P_{n A},
$$

onde $\delta P_{n A}$ é a perturbação não-adiabática intrínseca da pressão do fluido A. Utilizando a equação de balanço, (5.17), temos, no caso acoplado, que

$$
\delta P_{A}=c_{s A}^{2} \delta \rho_{A}+\left(c_{s A}^{2}-c_{a A}^{2}\right)\left[3 \mathcal{H}\left(1+\omega_{A}\right) \rho_{A}-a Q_{A}\right] \frac{\theta_{A}}{k^{2}} .
$$

Podemos escrever as equações (5.32) e (5.33) em função de quantidades que são invariantes de gauge. Para isso, faremos uso dos potenciais de Bardeen [10,44,45]

$$
\begin{aligned}
& \Psi=\psi-\frac{1}{a}\left[\left(-B+\frac{E^{\prime}}{2}\right) a\right]^{\prime}, \\
& \Phi=\phi-\frac{1}{6} \nabla^{2} E+\frac{a^{\prime}}{a}\left(B-\frac{E^{\prime}}{2}\right), \\
& D_{A}=\delta_{A}-\frac{\rho_{A}^{\prime}}{\rho_{A} \mathcal{H}}\left(\phi-\frac{1}{6} \nabla^{2} E\right), \\
& V_{A}=v_{A}-\frac{E^{\prime}}{2} .
\end{aligned}
$$

Podemos escolher um gauge em particular, por exemplo, o gauge newtoniano ou 
longitudinal que é dado por $E=0$ e $B=0$, de forma que temos $\Psi=\psi$ e $\Phi=\phi$. Contudo, no que segue, escreveremos nossas equações em função de invariantes de gauge.

A interação entre os componentes do setor escuro consistirá do caso mais geral, que é a combinação linear das densidades de energia da matéria e da energia escura,

$$
\begin{aligned}
& a Q_{c}=3 \mathcal{H}\left(\lambda_{1} \rho_{c}+\lambda_{2} \rho_{d}\right) \\
& a Q_{d}=-3 \mathcal{H}\left(\lambda_{1} \rho_{c}+\lambda_{2} \rho_{d}\right),
\end{aligned}
$$

onde $\lambda_{1}$ e $\lambda_{2}$ são pequenas constantes adimensionais. Em primeira ordem na teoria de perturbações, essa interação é dada por

$$
\delta Q_{c}^{0}=-3 H\left(\lambda_{1} \rho_{c}+\lambda_{2} \rho_{d}\right) \frac{\psi}{a}+3 H\left(\lambda_{1} \delta \rho_{c}+\lambda_{2} \delta \rho_{d}\right) \frac{1}{a}
$$

$\mathrm{e}$

$$
\delta Q_{d}^{0}=3 H\left(\lambda_{1} \rho_{c}+\lambda_{2} \rho_{d}\right) \frac{\psi}{a}-3 H\left(\lambda_{1} \delta \rho_{c}+\lambda_{2} \delta \rho_{d}\right) \frac{1}{a} .
$$

Tomamos aqui $\delta H=0$, pois assumimos que a taxa de expansão na interação é a taxa global de expansão.

Utilizando as variáveis independentes de gauge (5.42), as expressões para a interação (5.43) e a relação (5.41) nas equações (5.32) e (5.33), chegamos as equações, no espaço de Fourier, para a matéria escura $(c)$ e para a energia escura $(d)[4]$

$$
\begin{aligned}
& D_{c}^{\prime}=-k U_{c}+6 \mathcal{H}\left(\lambda_{1}+\lambda_{2} / r\right)-3\left(\lambda_{1}+\lambda_{2}\right) \Psi^{\prime}+3 \mathcal{H} \lambda_{2}\left(D_{d}-D_{c}\right) / r \\
& U_{c}^{\prime}=-\mathcal{H} U_{c}+k \Psi-3 \mathcal{H}\left(\lambda_{1}+\lambda_{2} / r\right) U_{c} \\
& D_{d}^{\prime}=-3 \mathcal{H} c_{s d}^{2}\left[D_{d}-\left[3\left(\lambda_{1} r+\lambda_{2}\right)+3(1+\omega)\right] \Phi\right]-3 \mathcal{H}\left(c_{s d}^{2}-c_{a d}^{2}\right) \\
&+\left[\frac{3 \mathcal{H} U_{d}}{k}-a Q_{d} \frac{U_{d}}{(1+\omega) \rho_{d} k}\right]-3 \mathcal{H} \omega\left[3\left(\lambda_{1} r+\lambda_{2}\right)+3(1+\omega)\right] \Phi \\
&+3 \mathcal{H} \omega D_{d}+3 \omega^{\prime} \Phi+3\left(\lambda_{1} r+\lambda_{2}\right) \Phi^{\prime}-k U_{d}-6 \Psi \mathcal{H}\left(\lambda_{1} r+\lambda_{2}\right) \\
&+ 3 \mathcal{H} \lambda_{1} r\left(D_{d}-D_{c}\right),
\end{aligned}
$$




$$
\begin{aligned}
U_{d}^{\prime} & =-\mathcal{H}(1-3 \omega) U_{d}+k c_{s d}^{2}\left[D_{d}-3\left[\left(\lambda_{1} r+\lambda_{2}\right)+(1+\omega)\right] \Phi\right] \\
& +\left(c_{s d}^{2}-c_{a d}^{2}\right) a Q_{d} \frac{U_{d}}{(1+\omega) \rho_{d}}+3\left(c_{s d}^{2}-c_{a d}^{2}\right) \mathcal{H} U_{d}+(1+\omega) k \Psi \\
& +3 \mathcal{H}\left(\lambda_{1} r+\lambda_{2}\right) U_{d} .
\end{aligned}
$$

Nessas equações, utilizamos a definição de $r=\rho_{c} / \rho_{d}$ e definimos $U=(1+\omega) V$.

Se assumirmos os valores (5.37) para a velocidade do som da energia escura, as equações (5.48) e (5.49) podem ser escritas como

$$
\begin{aligned}
D_{d}^{\prime} & =\left(-1+\omega+\lambda_{1} r\right) 3 \mathcal{H} D_{d}-9 \mathcal{H}^{2}(1-\omega)\left(1+\frac{\lambda_{1} r+\lambda_{2}}{1+\omega}\right) \frac{U_{d}}{k}-k U_{d} \\
& +9 \mathcal{H}(1-\omega)\left(\lambda_{1} r+\lambda_{2}+1+\omega\right) \Phi+3\left(\lambda_{1} r+\lambda_{2}\right) \Phi^{\prime}-6 \Psi \mathcal{H}\left(\lambda_{1} r+\lambda_{2}\right) \\
& -3 \mathcal{H} \lambda_{1} r D_{c}, \\
U_{d}^{\prime} & =2\left[1+\frac{3}{1+\omega}\left(\lambda_{1} r+\lambda_{2}\right)\right] \mathcal{H} U_{d}+k D_{d}-3 k\left(\lambda_{1} r+\lambda_{2}+1+\omega\right) \Phi \\
& +(1+\omega) k \Psi .
\end{aligned}
$$

Para resolvermos essas equações diferenciais, precisamos de condições iniciais para nossas variáveis. Consideraremos que nossas condições iniciais são adiabáticas, isto é, elas respeitam a expressão [46]

$$
\frac{D_{m}}{1-\lambda_{1}-\lambda_{2} / r}=\frac{D_{d}}{1+\omega+\lambda_{1} r+\lambda_{2}} .
$$

A perturbação da curvatura se relaciona com o contraste de densidade pela equação de Poisson $[4,47]$

$$
\Phi=\frac{4 \pi G a^{2} \sum \rho_{i}\left[D_{i}-\rho_{i}^{\prime} U_{i} / \rho_{i}\left(1+\omega_{i}\right) k\right]}{k^{2}-4 \pi G a^{2} \sum \rho_{i}^{\prime} / \mathcal{H}} .
$$

Já os potenciais $\Psi$ e $\Phi$ relacionam-se através de $\Psi=-\Phi$, visto que não estamos considerando a existência de tensores de estresse anisotrópicos para os fluidos do setor escuro.

Podemos, com as equações de balanço perturbadas e a relação entre a perturbação da curvatura e os contrastes de densidade dos componentes da matéria, estudar a estabilidade de tais equações diferenciais, o que determinará se elas são viáveis 
para o estudo da evolução cosmológica das perturbações lineares.

\subsubsection{Estabilidade da Perturbação de Curvatura}

A primeira observação que devemos fazer para entendermos o comportamento desse sistema de equações é que $\lambda_{1} r$ possui um comportamento do tipo scaling, isto é, essa quantidade é uma constante tanto no universo primordial quanto no tempo presente do universo. Essa conclusão resulta de nossa exigência para que $r$ seja uma constante, ou varie lentamente, durante parte da história do universo. Analiticamente, isso pode ser entendido ao inserirmos as equações de balanço

$$
\begin{aligned}
& \rho_{c}^{\prime}+3 \mathcal{H} \rho_{c}=3 \mathcal{H}\left(\lambda_{1} \rho_{c}+\lambda_{2} \rho_{d}\right), \\
& \rho_{d}^{\prime}+3 \mathcal{H} \rho_{d}(1+\omega)=-3 \mathcal{H}\left(\lambda_{1} \rho_{c}+\lambda_{2} \rho_{d}\right),
\end{aligned}
$$

em

$$
r^{\prime}=\frac{\rho_{c}^{\prime}}{\rho_{d}}-r \frac{\rho_{d}^{\prime}}{\rho_{d}}=0
$$

Resolvendo, como fizemos anteriormente, a equação quadrática correspondente para $r \lambda_{1}$, obtemos

$$
\begin{aligned}
& \left(r \lambda_{1}\right)_{1}=-\frac{1}{2}\left(\omega+\lambda_{1}+\lambda_{2}\right)+\frac{1}{2} \sqrt{\omega^{2}+2 \omega \lambda_{2}+2 \omega \lambda_{1}+\lambda_{2}^{2}-2 \lambda_{1} \lambda_{2}+\lambda_{1}^{2}} \\
& \left(r \lambda_{1}\right)_{2}=-\frac{1}{2}\left(\omega+\lambda_{1}+\lambda_{2}\right)-\frac{1}{2} \sqrt{\omega^{2}+2 \omega \lambda_{2}+2 \omega \lambda_{1}+\lambda_{2}^{2}-2 \lambda_{1} \lambda_{2}+\lambda_{1}^{2}} .
\end{aligned}
$$

Essas soluções implicam que para $\lambda_{1} \ll \lambda_{2}<-\omega$, temos que

$$
\begin{aligned}
& \left(r \lambda_{1}\right)_{1} \approx-\left(\omega+\lambda_{2}\right) \\
& \left(r \lambda_{1}\right)_{2} \approx-\frac{\lambda_{1} \lambda_{2}}{\omega+\lambda_{2}} \sim 0 .
\end{aligned}
$$

Essas duas raízes de $\lambda_{1} r$ são constantes no universo primordial ( $\rho_{c}$ dominante) e no atual universo ( $\rho_{d}$ dominante), respectivamente.

O comportamento scaling de $\lambda_{1} r$ influencia a perturbação de curvatura $\Phi$. Numericamente, foi encontrado em [48] que, quando $\omega>-1$ e $\lambda_{1} \neq 0, \Phi$ possui um comportamento explosivo no universo primordial. Isso ocorre independentemente do valor de $\lambda_{2}$. Contudo, quando se considera a interação entre os componentes do 
setor escuro como proporcional à densidade de energia escura e examina-se o caso que a equação de estado da energia escura é um pouco maior que -1, esse comportamento explosivo deixa de existir [48]. Um sistema estável também é encontrado quando se estende a discussão para o caso $\omega<-1$ [48].

Agora verificaremos esses resultados. Em [48] foi encontrado numericamente que os dois primeiros termos do lado direito das equações (5.50) e (5.51) contribuem muito mais do que os outros termos para a divergência. Usando $\xi_{1}$ e $\xi_{2}$ para representar os dois primeiros termos de (5.50), podemos escrever aproximadamente

$$
D_{d}^{\prime} \sim \xi_{1}+\xi_{2}
$$

onde

$$
\begin{aligned}
& \xi_{1}=\left(-1+\omega+\lambda_{1} r\right) 3 \mathcal{H} D_{d} \\
& \xi_{2}=-9 \mathcal{H}^{2}(1-\omega)\left(1+\frac{\lambda_{1} r+\lambda_{2}}{1+\omega}\right) \frac{U_{d}}{k} .
\end{aligned}
$$

Quando $\lambda_{2}=0, \lambda_{1} \neq 0$ e $-1<\omega<0, \lambda_{1} r \approx-\omega$ para o universo primordial, de modo que temos que $\xi_{2}$ é uma ordem de grandeza maior que $\xi_{1}$ e que $\xi_{1}+\xi_{2}>0$, o que causa um grande crescimento em $D_{d}$. Esse crescimento em $D_{d}$, por sua vez, implica em um crescimento em $\Phi$. Contudo, quando $\lambda_{1} \neq 0$ no caso em que $\omega<-1$ e no caso em que $\lambda_{1}=0$ com $\lambda_{2} \neq 0$, independentemente se $\omega<-1$ ou se $\omega>-1$, $\xi_{1}$ e $\xi_{2}$ são da mesma ordem e $\xi_{1}+\xi_{2}<0$, o que faz com que $D_{d}$ decresça com o tempo, impedindo o comportamento explosivo.

Para melhor entendermos a razão do comportamento explosivo na perturbação da métrica, faremos uma análise analítica. Mantendo somente os termos dominantes, temos as equações aproximadas

$$
\begin{aligned}
D_{d}^{\prime} & \approx\left(-1+\omega+\lambda_{1} r\right) 3 \mathcal{H} D_{d}-9 \mathcal{H}^{2}(1-\omega)\left(1+\frac{\lambda_{1} r+\lambda_{2}}{1+\omega}\right) \frac{U_{d}}{k} \\
U_{d}^{\prime} & \approx 2\left[1+\frac{3}{1+\omega}\left(\lambda_{1} r+\lambda_{2}\right)\right] \mathcal{H} U_{d}+k D_{d} .
\end{aligned}
$$

Considerando o caso em que a interação entre os componentes do setor escuro é proporcional à densidade de energia da matéria escura $\left(\lambda_{1} \neq 0\right.$ e $\left.\lambda_{2}=0\right)$ e notando que $\lambda_{1} r \sim-\omega$ no universo primordial, podemos simplificar as equações acima e obter 


$$
\begin{aligned}
D_{d}^{\prime} & \approx-3 \mathcal{H} D_{d}-9 \mathcal{H}^{2} \frac{1-\omega}{1+\omega} \frac{U_{d}}{k} \\
U_{d}^{\prime} & \approx 2 \frac{1-2 \omega}{1+\omega} \mathcal{H} U_{d}+k D_{d} .
\end{aligned}
$$

Podemos então encontrar uma equação diferencial de segunda ordem para $D_{d}$,

$$
D_{d}^{\prime \prime} \approx\left(2 \frac{\mathcal{H}^{\prime}}{\mathcal{H}}-\frac{1+7 \omega}{1+\omega} \mathcal{H}\right) D_{d}^{\prime}+3\left(\mathcal{H}^{\prime}-\mathcal{H}^{2}\right) D_{d}
$$

No período dominado pela radiação, temos $\mathcal{H} \sim \frac{1}{\eta}, \mathcal{H}^{\prime} \sim-\frac{1}{\eta^{2}}, \frac{\mathcal{H}^{\prime}}{\mathcal{H}} \sim-\frac{1}{\eta}$ e a equação (5.62) pode ser aproximada como

$$
D_{d}^{\prime \prime} \approx-3 \frac{1+3 \omega}{1+\omega} \frac{D_{d}^{\prime}}{\eta}-\frac{6}{\eta^{2}} D_{d} .
$$

A solução para essa equação é [48]

$$
D_{d} \approx C_{1} \eta^{r_{1}}+C_{2} \eta^{r_{2}}
$$

onde

$$
\begin{aligned}
& r_{1}=-\frac{1+4 \omega-\sqrt{-5-4 \omega+10 \omega^{2}}}{1+\omega} \\
& r_{2}=-\frac{1+4 \omega+\sqrt{-5-4 \omega+10 \omega^{2}}}{1+\omega} .
\end{aligned}
$$

Na figura 5.1, vemos que quando $-1<\omega<0$, tanto $r_{1}$ quanto $r_{2}$ são positivos, o que faz com que a perturbação $D_{d}$ aumente. Contudo, quando $\omega<-1$, tanto $r_{1}$ quanto $r_{2}$ são negativos; isso resulta no decaimento da perturbação $D_{d}$. Nesse caso, nenhuma divergência ocorre independentemente do valor de $\lambda_{1}$.

Esses resultados nos dizem que para uma equação de estado para a energia escura $\omega>-1$, um acoplamento entre a energia escura e a matéria escura proporcional a $\rho_{c}\left(\lambda_{1} \neq 0\right)$ levará a uma divergência na perturbação da curvatura. Contudo, essa divergência desaparece para $\omega<-1$.

Consideremos, agora, o caso em que a interação entre os componentes do setor escuro seja proporcional à densidade de energia escura $\left(\lambda_{1}=0\right.$ e $\left.\lambda_{2} \neq 0\right)$, nesse caso a equação (5.60) reduz-se a 

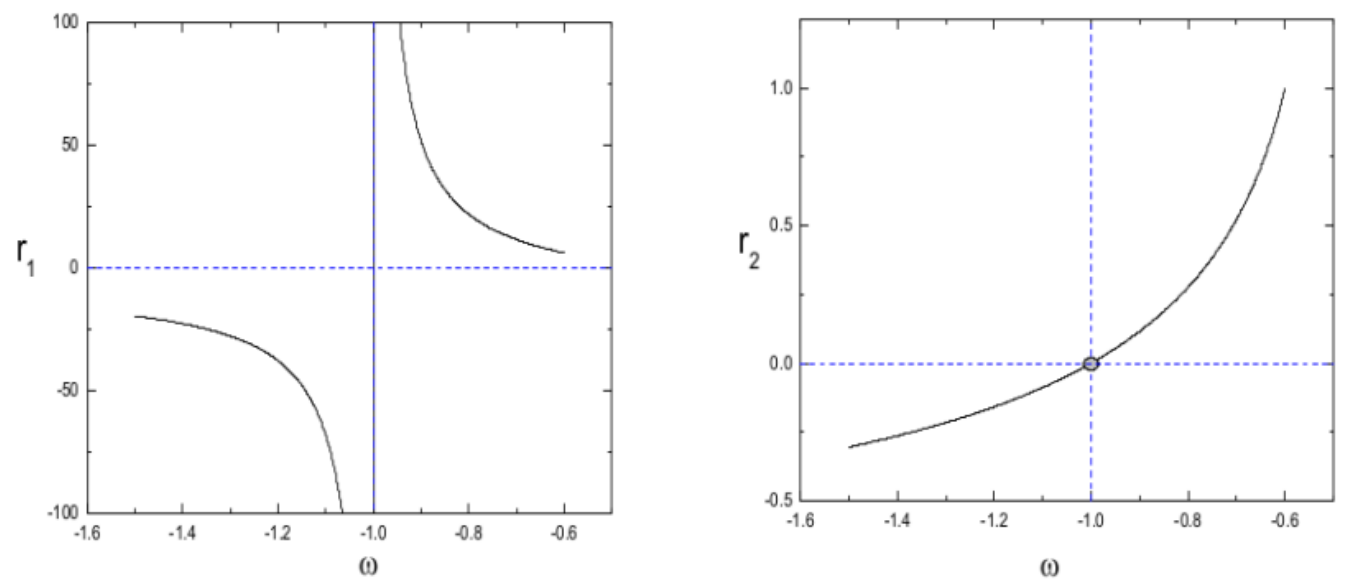

Figura 5.1: Comportamento dos índices $r_{1}$ e $r_{2}$ em função de $\omega$ [48]

$$
\begin{aligned}
D_{d}^{\prime} & \approx(-1+\omega) 3 \mathcal{H} D_{d}-9 \mathcal{H}^{2}(1-\omega)\left(1+\frac{\lambda_{2}}{1+\omega}\right) \frac{U_{d}}{k} \\
U_{d}^{\prime} & \approx 2\left(1+\frac{3 \lambda_{2}}{1+\omega}\right) \mathcal{H} U_{d}+k D_{d} .
\end{aligned}
$$

Podemos escrever a equação diferencial de segunda ordem para $D_{d}$ como

$$
\begin{aligned}
D_{d}^{\prime \prime} & =\left[\left(-1+3 \omega+\frac{6 \lambda_{2}}{1+\omega}\right) \mathcal{H}+2 \frac{\mathcal{H}^{\prime}}{\mathcal{H}}\right] D_{d}^{\prime} \\
& +3(1-\omega)\left[\mathcal{H}^{\prime}+\mathcal{H}^{2}\left(-1+\frac{3 \lambda_{2}}{1+\omega}\right)\right] D_{d}
\end{aligned}
$$

Na era dominada pela radiação, a equação acima se torna

$$
D_{d}^{\prime \prime}=\left(-3+3 \omega+\frac{6 \lambda_{2}}{1+\omega}\right) \frac{D_{d}^{\prime}}{\eta}+3(1-\omega)\left(-2+\frac{3 \lambda_{2}}{1+\omega}\right) \frac{D_{d}}{\eta^{2}} .
$$

Introduzindo as quantidades [48]

$$
\begin{aligned}
& \Gamma=3 \omega^{2}+\omega+6 \lambda_{2}-2, \\
& \Delta=9 \omega^{2}+30 \omega^{3}+13 \omega^{2}+\left(-28+12 \lambda_{2}\right) \omega+36 \lambda_{2}^{2}+12 \lambda_{2}-20,
\end{aligned}
$$

encontramos que, quando $\Delta>0$, 

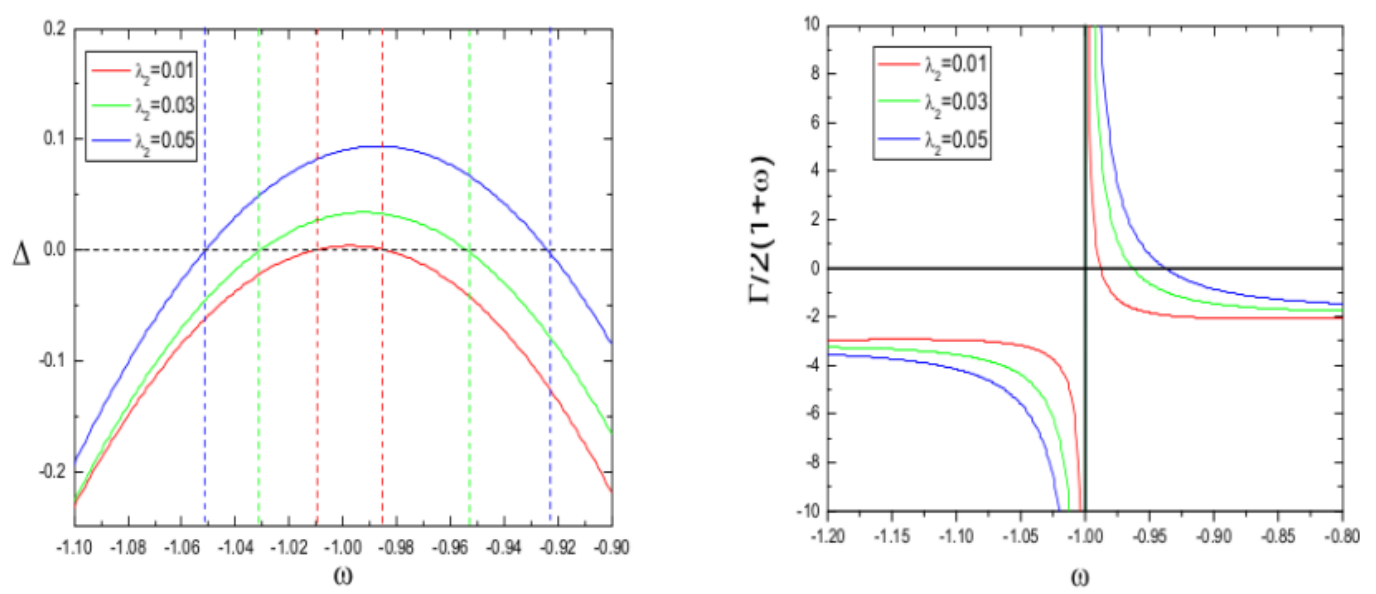

Figura 5.2: Comportamento de $\Delta$ e $\Gamma$ em função de $\omega$ [48]

$$
D_{d} \sim C_{1} \eta^{r_{1}}+C_{2} \eta^{r_{2}}
$$

onde

$$
\begin{aligned}
& r_{1}=\frac{1}{2} \frac{\Gamma}{1+\omega}+\frac{1}{2} \frac{\sqrt{\Delta}}{1+\omega}, \\
& r_{2}=\frac{1}{2} \frac{\Gamma}{1+\omega}-\frac{1}{2} \frac{\sqrt{\Delta}}{1+\omega} .
\end{aligned}
$$

Por outro lado, quando $\Delta<0$,

$$
D_{d} \sim C_{1} \eta^{\frac{1}{2} \frac{\Gamma}{1+\omega}} \cos \left(\frac{1}{2} \frac{\sqrt{|\Delta|}}{1+\omega} \ln \eta\right)+C_{2} \eta^{\frac{1}{2} \frac{\Gamma}{1+\omega}} \sin \left(\frac{1}{2} \frac{\sqrt{|\Delta|}}{1+\omega} \ln \eta\right)
$$

Na figura 5.2, podemos ver que $\Delta$ pode ser positivo somente na vizinhança de $\omega=-1$. Quando $\lambda_{2}$ é pequeno, o intervalo para que $\Delta$ seja positivo é pequeno. Vemos, também, que $\omega=-1$ é a singularidade central, pois ela leva a divergência de $r_{1}$ e de $r_{2}$ e, portanto, causa uma explosão na perturbação de densidade na equação (5.70). Quando $\omega>-1$ e $\Delta>0$, a explosão na densidade também pode ocorrer já que $\Gamma / 2(1+\omega)$ é positivo. Mas quando $\omega$ decresce além de $-1, \Delta$ se tornará negativo, assim como $\Gamma / 2(1+\omega)$, o que levará ao resultado convergente de (5.72). Quando $\omega<-1, \Gamma / 2(1+\omega)$ é sempre negativo, portanto, a densidade de perturbação decairá mesmo quando $\omega$ está perto de -1 , vindo da parte negativa da reta real, e $\Delta$ é pequeno e positivo.

A análise acima mostra que quando a interação entre os componentes do setor 
escuro é proporcional à densidade de energia escura $\left(\lambda_{2} \neq 0\right)$ a explosão na perturbação da curvatura não acontecerá para $\omega<-1$ constante. Para $\omega>-1$, quando o acoplamento é pequeno, a explosão também pode ser evitada no intervalo observacional dado para $\omega$. Contudo, existe a possibilidade que a divergência ocorra quando a interação é grande no intervalo observacionalmente permitido $\omega>-1$.

Concluímos, então, que quando a interação é proporcional à densidade da matéria escura e $\omega>-1$ temos a instabilidade da perturbação da métrica $\Psi$. Entretanto, se a interação for proporcional à densidade da energia escura e a constante de proporcionalidade $\lambda_{2}$ for pequena, o sistema é estável. O mesmo ocorre quando temos uma energia escura do tipo phantom com $\omega<-1$, independentemente do valor e do tipo de acoplamento. Esses resultados mostram que uma interação no setor escuro é cosmologicamente viável. 


\section{Capítulo 6}

\section{Interação no Setor Escuro no Formalismo Lagrangiano}

Neste capítulo, desenvolveremos duas alternativas ao modelo de fluidos interagentes no setor escuro. Para isso, usaremos dois formalismos distintos. Primeiramente, partiremos da lagrangiana que modifica a gravidade através de um termo geral que depende do escalar de Ricci, os chamados modelos de $f(R)$, e, através de uma transformação conforme, introduziremos um campo escalar canônico que interage com a matéria do universo. Depois, desenvolveremos um modelo efetivo para a interação no setor escuro dentro do formalismo da teoria quântica de campos. Nesse caso, a energia escura será representada por um campo escalar canônico e a matéria escura por um campo fermiônico de spin- $\frac{1}{2}$. A interação será dada por um termo de Yukawa na lagrangiana.

\subsection{Modelo para a Energia Escura Interagindo com a Matéria Escura Oriundo de Modelos de $f(R)$}

Nesta seção, analisaremos um modelo de interação no setor escuro que utiliza um campo escalar para representar a energia escura. Esse modelo consiste em uma reinterpretação dos modelos de gravidade modificada do tipo $f(R)$ utilizando a linguagem da teoria de campos. Assim, primeiramente discutiremos os modelos de $f(R)$ no formalismo métrico e estudaremos a transformação conforme que permite reinterpretar os modelos de $f(R)$ como modelos de interação entre energia e matéria escura. Com as equações de balanço para o setor escuro obtidas através desse formalismo, discutiremos o modelo de quintessência acoplada resultante. 


\subsubsection{Modelos de $f(R)$ no Formalismo Métrico}

Podemos generalizar a ação de Einstein-Hilbert, (4.39), para um forma mais geral desde que a ação seja composta por invariantes de curvatura. A ação de EinsteinHilbert pode, portanto, ser interpretada como o limite para a gravidade em escalas como a do nosso sistema solar, onde a Relatividade Geral é compatível com as observações. Dessa forma, podemos escrever a ação quadridimensional para a gravidade como sendo dependente de uma função do escalar de Ricci que será denotada por $f(R)$. Assim, temos que [49]

$$
S=\frac{1}{2 \kappa^{2}} \int \mathrm{d}^{4} x \sqrt{-g} f(R)+\int \mathrm{d}^{4} x \mathcal{L}_{M}\left(g_{\mu \nu}, \Psi_{M}\right),
$$

onde $\kappa^{2}=8 \pi G, g$ é o determinante da métrica $g_{\mu \nu}$ e $\mathcal{L}_{M}$ é a lagrangiana da matéria que depende de $g_{\mu \nu}$ e dos campos de matéria $\Psi_{M}$. O escalar de Ricci $R$ é definido como $R=g^{\mu \nu} R_{\mu \nu}$, onde o tensor de Ricci $R_{\mu \nu}$ é

$$
R_{\mu \nu}=R_{\mu \alpha \nu}^{\alpha}=\partial_{\lambda} \Gamma_{\mu \nu}^{\lambda}-\partial_{\mu} \Gamma_{\lambda \nu}^{\lambda}+\Gamma_{\mu \nu}^{\lambda} \Gamma_{\rho \lambda}^{\rho}-\Gamma_{\nu \rho}^{\lambda} \Gamma_{\mu \lambda}^{\rho} .
$$

Os coeficientes de conexão $\Gamma_{\beta \gamma}^{\alpha}$ são dados em termos da métrica $g_{\mu \nu}$ por

$$
\Gamma_{\beta \gamma}^{\alpha}=\frac{1}{2} g^{\alpha \lambda}\left(\frac{\partial g_{\gamma \lambda}}{\partial x^{\beta}}+\frac{\partial g_{\lambda \beta}}{\partial x^{\gamma}}-\frac{\partial g_{\beta \gamma}}{\partial x^{\lambda}}\right)
$$

As equações de campo que generalizam as equações de Einstein podem ser derivadas ao variarmos a ação (6.1) com respeito a $g_{\mu \nu}$,

$$
\Sigma_{\mu \nu} \equiv F(R) R_{\mu \nu}-\frac{1}{2} f(R) g_{\mu \nu}-\nabla_{\mu} \nabla_{\nu} F(R)+g_{\mu \nu} \square F(R)=\kappa^{2} T_{\mu \nu}^{(M)},
$$

onde $F(R) \equiv \partial f / \partial R$. $T_{\mu \nu}^{(M)}$ é o tensor de energia-momento dos campos de matéria definido como

$$
T_{\mu \nu}^{(M)}=-\frac{2}{\sqrt{-g}} \frac{\delta \mathcal{L}_{M}}{\delta g^{\mu \nu}} .
$$

Esse tensor satisfaz a equação de continuidade

$$
\nabla^{\mu} T_{\mu \nu}^{(M)}=0
$$

$\Sigma_{\mu \nu}$ também respeita essa identidade, $\nabla^{\mu} \Sigma_{\mu \nu}=0$, graças às identidades de Bianchi [6]. O traço da equação (6.4) é 


$$
3 \square F(R)+F(R) R-2 f(R)=\kappa^{2} T,
$$

onde $T=g^{\mu \nu} T_{\mu \nu}^{(M)}$ e $\square F=(1 / \sqrt{-g}) \partial_{\mu}\left(\sqrt{-g} g^{\mu \nu} \partial_{\nu} F\right)$.

A gravidade de Einstein, sem a presença de uma constante cosmológica, corresponde a $f(R)=R$ e $F(R)=1$, de forma que o termo $\square F(R)$ na equação (6.7) anula-se. Nesse caso, temos que $R=-\kappa^{2} T$ e, portanto, o escalar de Ricci é diretamente determinado pela matéria (o traço $T$ ).

As equações de campo (6.4) podem ser escritas na forma [50]

$$
G_{\mu \nu}=\kappa^{2}\left(T_{\mu \nu}^{(M)}+T_{\mu \nu}^{(D)}\right)
$$

onde $G_{\mu \nu} \equiv R_{\mu \nu}-(1 / 2) g_{\mu \nu} R$ e

$$
\kappa^{2} T_{\mu \nu}^{(D)} \equiv g_{\mu \nu}(f-R) / 2+\nabla_{\mu} \nabla_{\nu} F-g_{\mu \nu} \square F+(1-F) R_{\mu \nu} .
$$

Como $\nabla^{\mu} G_{\mu \nu}=0$ e $\nabla^{\mu} T_{\mu \nu}^{(M)}=0$, temos que

$$
\nabla^{\mu} T_{\mu \nu}^{(D)}=0
$$

Portanto, a equação de continuidade é válida também para o tensor de energiamomento efetivo $T_{\mu \nu}^{(D)}$.

Considerando um espaço-tempo de FRW espacialmente plano com um fator de escala $a(t)$, temos uma métrica do tipo

$$
\mathrm{d} s^{2}=g_{\mu \nu} \mathrm{d} x^{\mu} \mathrm{d} x^{\nu}=-\mathrm{d} t^{2}+a^{2}(t) \mathrm{d} \mathbf{x}^{2} .
$$

Para essa métrica, o escalar de Ricci é dado por

$$
R=6\left(2 H^{2}+\dot{H}\right)
$$

onde $H=\dot{a} / a$ é o parâmetro de Hubble.

O tensor de energia-momento da matéria é dado por $T_{\nu}^{\mu(M)}=\operatorname{diag}\left(-\rho_{M}, P_{M}, P_{M}\right.$, $\left.P_{M}\right)$. Assim, as equações de campo (6.4), no universo de fundo de FRW plano, são

$$
\begin{gathered}
3 F H^{2}=(F R-f) / 2-3 H \dot{F}+\kappa^{2} \rho_{M} \\
-2 F \dot{H}=\ddot{F}-H \dot{F}+\kappa^{2}\left(\rho_{M}+P_{M}\right),
\end{gathered}
$$

Essas equações generalizam as equações de Friedmann para uma gravidade $f(R)$. O fluido perfeito correspondente à parte material do universo satisfaz a equação de 
continuidade

$$
\dot{\rho}_{M}+3 H\left(\rho_{M}+P_{M}\right)=0 .
$$

\subsubsection{Equivalência com a Teoria de Brans-Dicke}

Os modelos de $f(R)$ no formalismo métrico podem ser reescritos na forma da teoria de Brans-Dicke com um potencial para o campo escalar efetivo [49]. Consideraremos a ação para um campo escalar $\chi$,

$$
S=\frac{1}{2 \kappa^{2}} \int \mathrm{d}^{4} x \sqrt{-g}\left[f(\chi)+f_{, \chi}(\chi)(R-\chi)\right]+\int \mathrm{d}^{4} x \mathcal{L}_{M}\left(g_{\mu \nu}, \Psi_{M}\right) .
$$

Variando essa ação com respeito a $\chi$, obtemos que

$$
f_{, \chi \chi}(\chi)(R-\chi)=0
$$

Se tivermos que $f_{, \chi \chi}(\chi) \neq 0$, precisamos que $\chi=R$, de modo que a ação (6.16) recupera a ação (6.1) na gravidade de $f(R)$. Assim, se definirmos

$$
\varphi \equiv f_{, \chi}(\chi)
$$

a ação (6.16) pode ser expressa como

$$
S=\int \mathrm{d}^{4} x \sqrt{-g}\left[\frac{1}{2 \kappa^{2}} \varphi R-U(\varphi)\right]+\int \mathrm{d}^{4} x \mathcal{L}_{M}\left(g_{\mu \nu}, \Psi_{M}\right),
$$

onde $U(\varphi)$ é um potencial dado por

$$
U(\varphi)=\frac{\chi(\varphi) \varphi-f(\chi(\varphi))}{2 \kappa^{2}} .
$$

Por outro lado, a ação da teoria de Brans-Dicke com um potencial $U(\varphi)$ é dada por $[49,51]$

$$
S=\int \mathrm{d}^{4} x \sqrt{-g}\left[\frac{1}{2} \varphi R-\frac{\omega_{\mathrm{BD}}}{2 \varphi}(\nabla \varphi)^{2}-U(\varphi)\right]+\int \mathrm{d}^{4} x \mathcal{L}_{M}\left(g_{\mu \nu}, \Psi_{M}\right),
$$

onde fizemos $\kappa^{2}=1, \omega_{\mathrm{BD}}$ é o parâmetro de Brans-Dicke e $(\nabla \varphi)^{2}=g^{\mu \nu} \partial_{\mu} \varphi \partial_{\nu} \varphi$. Comparando as equações (6.19) e (6.21), vemos que os modelos de $f(R)$ no formalismo métrico são equivalentes a uma teoria de Brans-Dicke com o parâmetro $\omega_{\mathrm{BD}}=0$. 
Motivados por essa equivalência, podemos buscar uma interpretação para a gravidade $f(R)$ utilizando a linguagem da teoria de campos e da Relatividade Geral de Einstein.

\subsubsection{Transformação Conforme}

A ação (6.1) da gravidade de $f(R)$ corresponde a um funcional não-linear de $R$. É possível derivar uma ação no referencial de Einstein - o referencial de Einstein é definido como o referencial em que a ação é linear no escalar de Ricci $R$ - através de uma transformação conforme $[52,53]$

$$
\tilde{g}_{\mu \nu}=\Omega^{2} g_{\mu \nu},
$$

onde $\Omega^{2}$ é o fator conforme e um til representa quantidades no referencial de Einstein. O escalares de Ricci $R$ e $\tilde{R}$, nos diferentes referenciais, possuem a relação

$$
R=\Omega^{2}\left(\tilde{R}+6 \tilde{\square} \omega-6 \tilde{g}^{\mu \nu} \partial_{\mu} \omega \partial_{\nu} \omega\right)
$$

onde

$$
\omega \equiv \ln \Omega, \quad \partial_{\mu} \omega \equiv \frac{\partial \omega}{\partial \tilde{x}^{\mu}}, \quad \tilde{\square} \omega \equiv \frac{1}{\sqrt{-\tilde{g}}} \partial_{\mu}\left(\sqrt{-\tilde{g}} \tilde{g}^{\mu \nu} \partial_{\nu} \omega\right)
$$

Podemos reescrever a ação (6.1) na forma

$$
S=\int \mathrm{d}^{4} x \sqrt{-g}\left(\frac{1}{2 \kappa^{2}} F R-U\right)+\int \mathrm{d}^{4} x \mathcal{L}_{M}\left(g_{\mu \nu}, \Psi_{M}\right),
$$

onde

$$
U=\frac{F R-f}{2 \kappa^{2}} .
$$

Usando a equação (6.23) e a relação $\sqrt{-g}=\Omega^{-4} \sqrt{-\tilde{g}}$, a ação (6.25) pode ser escrita como

$$
\begin{aligned}
S=\int \mathrm{d}^{4} x & \sqrt{-\tilde{g}}\left[\frac{1}{2 \kappa^{2}} F \Omega^{-2}\left(\tilde{R}+6 \tilde{\square} \omega-6 \tilde{g}^{\mu \nu} \partial_{\mu} \omega \partial_{\nu} \omega\right)-\Omega^{-4} U\right] \\
& +\int \mathrm{d}^{4} x \mathcal{L}_{M}\left(\Omega^{-2} \tilde{g}_{\mu \nu}, \Psi_{M}\right) .
\end{aligned}
$$

Obtemos a ação no referencial de Einstein, isto é, ação linear em $\tilde{R}$ ), se fizermos a escolha 


$$
\Omega^{2}=F
$$

Essa escolha é consistente se $F>0$. Agora, introduziremos um novo campo escalar $\phi$, que é definido por

$$
\kappa \phi \equiv \sqrt{3 / 2} \ln F
$$

Da definição de $\omega$ na equação (6.24), temos que $\omega=\kappa \phi / \sqrt{6}$. Usando a equação (6.24), a integral $\int \mathrm{d}^{4} x \sqrt{-\tilde{g}} \tilde{\square} \omega$ é idêntica a zero devido ao teorema de Gauss. Desse modo, a ação no referencial de Einstein é

$$
\begin{aligned}
& S_{E}=\int \mathrm{d}^{4} x \sqrt{-\tilde{g}}\left[\frac{1}{2 \kappa^{2}} \tilde{R}-\frac{1}{2} \tilde{g}^{\mu \nu} \partial_{\mu} \phi \partial_{\nu} \phi-V(\phi)\right] \\
&+\int \mathrm{d}^{4} x \mathcal{L}_{M}\left(F^{-1}(\phi) \tilde{g}_{\mu \nu}, \Psi_{M}\right),
\end{aligned}
$$

onde

$$
V(\phi)=\frac{U}{F^{2}}=\frac{F R-f}{2 \kappa^{2} F^{2}} .
$$

Assim, a densidade lagrangiana do campo $\phi$ é dada por $\mathcal{L}_{\phi}=-\frac{1}{2} \tilde{g}^{\mu \nu} \partial_{\mu} \phi \partial_{\nu} \phi-V(\phi)$, com o tensor de energia-momento dado por

$$
\tilde{T}_{\mu \nu}^{(\phi)}=-\frac{2}{\sqrt{-\tilde{g}}} \frac{\delta\left(\sqrt{-\tilde{g}} \mathcal{L}_{\phi}\right)}{\delta \tilde{g}^{\mu \nu}}=\partial_{\mu} \phi \partial_{\nu} \phi-\tilde{g}_{\mu \nu}\left[\frac{1}{2} \tilde{g}^{\alpha \beta} \partial_{\alpha} \phi \partial_{\beta} \phi+V(\phi)\right]
$$

O fator conforme $\Omega^{2}=F=\exp (\sqrt{2 / 3} \kappa \phi)$ depende do campo escalar. Na ação da matéria (6.30), vemos que o campo escalar $\phi$ é diretamente acoplado à matéria no referencial de Einstein. Variando a ação (6.30) com respeito ao campo escalar $\phi$ temos que

$$
-\partial_{\mu}\left(\frac{\partial\left(\sqrt{-\tilde{g}} \mathcal{L}_{\phi}\right)}{\partial\left(\partial_{\mu} \phi\right)}\right)+\frac{\partial\left(\sqrt{-\tilde{g}} \mathcal{L}_{\phi}\right)}{\partial \phi}+\frac{\partial \mathcal{L}_{M}}{\partial \phi}=0
$$

isto é,

$$
\tilde{\square} \phi-V_{, \phi}+\frac{1}{\sqrt{-\tilde{g}}} \frac{\partial \mathcal{L}_{M}}{\partial \phi}=0 .
$$

Usando a equação (6.22) e as relações $\sqrt{-\tilde{g}}=F^{2} \sqrt{-g}$ e $\tilde{g}^{\mu \nu}=F^{-1} g^{\mu \nu}$, o tensor de energia-momento da matéria torna-se 


$$
\tilde{T}_{\mu \nu}^{(M)}=-\frac{2}{\sqrt{-\tilde{g}}} \frac{\delta \mathcal{L}_{M}}{\delta \tilde{g}^{\mu \nu}}=\frac{T_{\mu \nu}^{(M)}}{F} .
$$

O tensor de energia-momento do fluido perfeito no referencial de Einstein é dado, portanto, por

$$
\begin{aligned}
\tilde{T}_{\nu}^{\mu(M)} & =\operatorname{diag}\left(-\tilde{\rho}_{M}, \tilde{P}_{M}, \tilde{P}_{M}, \tilde{P}_{M}\right) \\
& =\operatorname{diag}\left(-\rho_{M} / F^{2}, P_{M} / F^{2}, P_{M} / F^{2}, P_{M} / F^{2}\right) .
\end{aligned}
$$

A derivada da densidade lagrangiana $\mathcal{L}_{M}=\mathcal{L}_{M}\left(g_{\mu \nu}\right)=\mathcal{L}_{M}\left(F^{-1}(\phi) \tilde{g}_{\mu \nu}\right)$ com respeito a $\phi$ é

$$
\frac{\partial \mathcal{L}_{M}}{\partial \phi}=\frac{\delta \mathcal{L}_{M}}{\delta g^{\mu \nu}} \frac{\partial g^{\mu \nu}}{\partial \phi}=\frac{1}{F(\phi)} \frac{\delta \mathcal{L}_{M}}{\delta \tilde{g}^{\mu \nu}} \frac{\partial\left(F(\phi) \tilde{g}^{\mu \nu}\right)}{\partial \phi}=-\sqrt{-\tilde{g}} \frac{F_{, \phi}}{2 F} \tilde{T}_{\mu \nu}^{(M)} \tilde{g}^{\mu \nu}
$$

O acoplamento entre o campo escalar e a matéria pode ser quantificado pela quantidade [54]

$$
Q \equiv-\frac{F_{, \phi}}{2 \kappa F}=-\frac{1}{\sqrt{6}}
$$

que é uma constante na gravidade de $f(R)$. Temos, então, que

$$
\frac{\partial \mathcal{L}_{M}}{\partial \phi}=\sqrt{-\tilde{g}} \kappa Q \tilde{T}
$$

onde $\tilde{T}=\tilde{g}_{\mu \nu} \tilde{T}^{\mu \nu(M)}=-\tilde{\rho}_{M}+3 \tilde{P}_{M}$. Substituindo a equação (6.39) na equação (6.34), obtemos a equação de campo no referencial de Einstein,

$$
\tilde{\square} \phi-V_{, \phi}+\kappa Q \tilde{T}=0
$$

Isso mostra que o campo escalar $\phi$ é diretamente acoplado à matéria, exceto a radiação, já que, nesse caso, temos $\tilde{T}=0$.

No referencial de Jordan - referencial em que a ação da matéria é linear em $g_{\mu \nu}$-, o espaço-tempo de FRW plano é dado pela métrica (6.11). A métrica no referencial de Einstein, por sua vez, é dada por

$$
\begin{aligned}
\mathrm{d} \tilde{s}^{2}=\Omega^{2} \mathrm{~d} s^{2} & =F\left(-\mathrm{d} t^{2}+a^{2}(t) \mathrm{d} \mathbf{x}^{2}\right) \\
& =-\mathrm{d} \tilde{t}^{2}+\tilde{a}^{2}(\tilde{t}) \mathrm{d} \mathbf{x}^{2}
\end{aligned}
$$


o que nos leva as relações (para $F>0$ )

$$
\mathrm{d} \tilde{t}=\sqrt{F} \mathrm{~d} t, \quad \tilde{a}=\sqrt{F} a
$$

onde

$$
F=e^{-2 Q \kappa \phi}
$$

Note que a equação (6.43) é oriunda da integração da equação (6.38) para $Q$ constante. A equação de campo (6.40) pode ser expressa como

$$
\frac{\mathrm{d}^{2} \phi}{\mathrm{d} \tilde{t}^{2}}+3 \tilde{H} \frac{\mathrm{d} \phi}{\mathrm{d} \tilde{t}}+V_{, \phi}=-\kappa Q\left(\tilde{\rho}_{M}-3 \tilde{P}_{M}\right)
$$

onde

$$
\tilde{H} \equiv \frac{1}{\tilde{a}} \frac{\mathrm{d} \tilde{a}}{\mathrm{~d} \tilde{t}}=\frac{1}{\sqrt{F}}\left(H+\frac{\dot{F}}{2 F}\right) .
$$

Definindo a densidade de energia $\tilde{\rho}_{\phi}=\frac{1}{2}(\mathrm{~d} \phi / \mathrm{d} \tilde{t})^{2}+V(\phi)$ e a pressão $\tilde{P}_{\phi}=\frac{1}{2}(\mathrm{~d} \phi / \mathrm{d} \tilde{t})^{2}-$ $V(\phi)$, a equação (6.44) pode ser escrita como

$$
\frac{\mathrm{d} \tilde{\rho}_{\phi}}{\mathrm{d} \tilde{t}}+3 \tilde{H}\left(\tilde{\rho}_{\phi}+\tilde{P}_{\phi}\right)=-\kappa Q\left(\tilde{\rho}_{M}-3 \tilde{P}_{M}\right) \frac{\mathrm{d} \phi}{\mathrm{d} \tilde{t}} .
$$

Utilizando a transformação (6.42) juntamente com $\rho_{M}=F^{2} \tilde{\rho}_{M}, P_{M}=F^{2} \tilde{P}_{M}$ e $H=F^{1 / 2}[\tilde{H}-(\mathrm{d} F / \mathrm{d} \tilde{t}) / 2 F]$, a equação de continuidade (6.15) pode ser transformada em

$$
\frac{\mathrm{d} \tilde{\rho}_{M}}{\mathrm{~d} \tilde{t}}+3 \tilde{H}\left(\tilde{\rho}_{M}+\tilde{P}_{M}\right)=\kappa Q\left(\tilde{\rho}_{M}-3 \tilde{P}_{M}\right) \frac{\mathrm{d} \phi}{\mathrm{d} \tilde{t}} .
$$

As equações (6.46) e (6.47) mostram que o campo escalar e a matéria interagem entre si, enquanto a densidade de energia total $\tilde{\rho}_{T}=\tilde{\rho}_{\phi}+\tilde{\rho}_{M}$ e a pressão total $\tilde{P}_{T}=\tilde{P}_{\phi}+\tilde{P}_{M}$ satisfazem a equação de continuidade $\mathrm{d} \tilde{\rho}_{T} / \mathrm{d} \tilde{t}+3 \tilde{H}\left(\tilde{\rho}_{T}+\tilde{P}_{T}\right)=0$. Mais geralmente, as equações (6.46) e (6.47) podem ser expressas em termos dos tensores de energia-momento definidos nas equações (6.32) e (6.35) [55],

$$
\tilde{\nabla}_{\mu} \tilde{T}_{\nu}^{\mu(\phi)}=-\kappa Q \tilde{T} \tilde{\nabla}_{\nu} \phi, \quad \tilde{\nabla}_{\mu} \tilde{T}_{\nu}^{\mu(M)}=\kappa Q \tilde{T} \tilde{\nabla}_{\nu} \phi
$$

\subsubsection{Quintessência Acoplada}

Concluímos a seção anterior com equações de balanço para a energia escura interagente. A energia escura sendo representada por um campo escalar e a matéria 
do universo sendo representada por um fluido ideal. A constante de interação $Q$ é dada pelo valor (6.38) para um modelo em que a origem da interação entre a energia escura e a matéria do universo é o fato que a gravidade é dada por um modelo $f(R)$. Podemos generalizar essa constante não fixando seu valor, mas sim tentando obtê-la através de observações cosmológicas. Esse tipo de interação pode ser motivado por teorias que vão além do modelo padrão da física de partículas $[18,56]$. Nesta seção, postularemos, por simplicidade, que a energia escura interage somente com a matéria escura e não com a matéria bariônica. Assim, evitaremos o problema conhecido como o problema da quinta força $[18,57]$. Desse modo, partindo das equações (6.48), estudaremos o comportamento da quintessência acoplada à matéria em uma métrica de FRW; primeiramente no universo de fundo e depois no o universo perturbado linearmente. No que segue adotaremos as unidades em que $\kappa=1$.

\section{Universo de Fundo}

Para a interação dada pela equação (6.48), o campo escalar $\phi$ e a matéria escura obedecem as seguintes equações de movimento, respectivamente, em um fundo de FRW plano,

$$
\begin{gathered}
\dot{\rho}_{\phi}+3 H\left(\rho_{\phi}+P_{\phi}\right)=-Q \rho_{c} \dot{\phi}, \\
\dot{\rho}_{c}+3 H \rho_{c}=Q \rho_{c} \dot{\phi},
\end{gathered}
$$

Considerando a equação de Friedmann para o universo tardio, no qual tanto a radiação quanto a matéria bariônica pode ser desprezada,

$$
3 H^{2}=\rho_{\phi}+\rho_{c}
$$

temos um sistema fechado de equações para o universo de fundo. Como $\rho_{\phi}=$ $\frac{1}{2} \dot{\phi}^{2}+V(\phi)$ e $P_{\phi}=\frac{1}{2} \dot{\phi}^{2}-V(\phi)$, a equação (6.49) pode ser reescrita como

$$
\ddot{\phi}+3 H \dot{\phi}+V_{, \phi}=-Q \rho_{c}
$$

\section{Universo Perturbado Linearmente}

Agora, podemos considerar um universo perturbado linearmente e, assim, obter as equações que determinam a evolução das perturbações da métrica, do campo e da matéria. Para isso, utilizaremos o gauge síncrono, de modo que a métrica pode ser escrita como $[10,55]$ 


$$
\mathrm{d} s^{2}=-a^{2}(\tau) \mathrm{d} \tau^{2}+a^{2}(\tau)\left[\left(1+\frac{1}{3} h\right) \delta_{i j}+D_{i j} \eta\right] \mathrm{d} x^{i} \mathrm{~d} x^{j},
$$

onde estamos considerando somente as perturbações escalares. Como essa métrica está escrita em função do tempo conforme, $\tau$, adiante toda derivada "temporal" será em relação ao tempo conforme. O gauge síncrono foi escolhido, pois o código CAMB, que é utilizado para resolver equações diferenciais cosmológicas, foi escrito utilizando o gauge síncrono.

Assim, definindo o campo escalar como composto por uma parte homogênea e uma parte perturbada: $\phi(t)+\varphi(\mathbf{x}, t)$, o contraste de densidade como $\delta=\delta \rho / \rho$ e a divergência da velocidade como $\theta=\nabla_{i} v^{i}$, obtemos das equações (6.48) as equações para a matéria com equação de estado geral $\omega_{M}$,

$$
\begin{aligned}
\dot{\delta}_{M} & =3(\mathcal{H}+Q \dot{\phi})\left(\omega_{M}-\frac{\delta P_{M}}{\delta \rho_{M}}\right) \delta_{M}-\left(1+\omega_{M}\right)\left(\theta_{M}+\frac{\dot{h}}{2}\right) \\
+ & Q\left(1-3 \omega_{M}\right) \dot{\varphi}+Q^{\prime} \dot{\phi} \varphi\left(1-3 \omega_{M}\right), \\
\dot{\theta}_{M} & =-\mathcal{H}\left(1-3 \omega_{M}\right) \theta_{M}-\frac{\dot{\omega}_{M}}{1+\omega_{M}} \theta_{M}+\frac{\delta P_{M} / \delta \rho_{M}}{1+\omega_{M}} k^{2} \theta_{M} \\
& +Q \frac{1-3 \omega_{M}}{1+\omega_{M}} k^{2} \varphi-Q\left(1-3 \omega_{M}\right) \dot{\phi} \theta_{M},
\end{aligned}
$$

onde $\delta P_{M}$ é a perturbação da pressão da matéria e $Q^{\prime} \equiv \partial Q / \partial \phi$. Considerando a matéria como sendo dada pela matéria escura $\left(\omega_{c}=0\right.$ e $\left.\delta P_{c}=0\right)$, o que é uma boa aproximação para o universo tardio, e a constante de acoplamento independente de $\phi\left(Q^{\prime}=0\right)$, temos que

$$
\begin{gathered}
\dot{\delta}_{c}=-\theta_{c}-\dot{h} / 2+Q \dot{\varphi}, \\
\dot{\theta}_{c}=-\mathcal{H} \theta_{c}-Q \dot{\phi} \theta_{c}+k^{2} Q \varphi .
\end{gathered}
$$

Para o campo escalar, obtemos de (6.48) a equação de Klein-Gordan perturbada, que é dada por

$$
\ddot{\varphi}+2 \mathcal{H} \dot{\varphi}+k^{2} \varphi+a^{2} V^{\prime \prime} \varphi+\dot{h} \dot{\phi} / 2=-a^{2} Q \rho_{c} \delta_{c},
$$

onde $V^{\prime \prime}=\partial^{2} V / \partial \phi^{2}$. 
Precisamos, por fim, de condições iniciais para o campo escalar perturbado e sua derivada temporal para que possamos resolver a equação (6.58). As análises das anisotropias da temperatura da radiação cósmica de fundo mostram consistentemente que é razoável supor que as perturbações das densidades da matéria eram inicialmente relacionadas através de condições adiabáticas. Por essa razão, proporemos para as perturbações da energia escura e da matéria escura condições iniciais adiabáticas [58],

$$
\frac{\delta \rho_{\phi}}{\rho_{\phi}+P_{\phi}}=\frac{\delta \rho_{c}}{\rho_{c}+P_{c}}
$$

Contudo, durante a evolução do universo, perturbações de isocurvatura serão produzidas e a condição adiabática não será futuramente conservada, mesmo para modos de larga escala, devido à presença de um acoplamento não mínimo $[48,59]$. Assim, a escolha da condição adiabática inicial não é determinante para a evolução de nosso sistema de equações. De qualquer maneira, para o campo escalar, temos que

$$
\rho_{\phi}=\frac{\dot{\phi}^{2}}{2 a^{2}}+V(\phi), \quad P_{\phi}=\frac{\dot{\phi}^{2}}{2 a^{2}}-V(\phi) .
$$

Por sua vez, a densidade perturbada e a pressão perturbada do campo escalar são dadas por

$$
\delta \rho_{\phi}=\frac{\dot{\phi} \dot{\varphi}}{a^{2}}+\frac{\mathrm{d} V}{\mathrm{~d} \phi} \varphi, \quad \delta P_{\phi}=\frac{\dot{\phi} \dot{\varphi}}{a^{2}}-\frac{\mathrm{d} V}{\mathrm{~d} \phi} \varphi .
$$

Desse modo, a equação (6.59) pode ser escrita como

$$
\frac{\dot{\phi} \dot{\varphi}+a^{2} \frac{\mathrm{d} V}{\mathrm{~d} \phi} \varphi}{\dot{\phi}^{2}}=\delta_{c} .
$$

Contudo, essa equação não é suficiente para determinarmos inequivocamente $\varphi$ e $\dot{\varphi}$, para isso precisamos de mais uma condição inicial. Baseados nas escolhas de condições iniciais utilizadas em estudos do universo inflacionário, consideraremos que a entropia intrínseca do campo escalar seja zero, isto é, que tenhamos $[58,60]$

$$
\frac{\delta \rho_{\phi}}{\rho_{\phi}}-\frac{\delta P_{\phi}}{P_{\phi}}=0
$$

Essa equação implica em

$$
\frac{\dot{\phi} \dot{\varphi}+a^{2} \frac{\mathrm{d} V}{\mathrm{~d} \phi} \varphi}{\frac{\dot{\phi}^{2}}{2}+a^{2} V}=\frac{\dot{\phi} \dot{\varphi}-a^{2} \frac{\mathrm{d} V}{\mathrm{~d} \phi} \varphi}{\frac{\dot{\phi}^{2}}{2}-a^{2} V} .
$$

Agora, resolvendo para $\varphi$ e $\dot{\varphi}$ as equações (6.62) e (6.64), obtemos as condições 
iniciais para o campo escalar perturbado,

$$
\varphi=\frac{2 \delta_{c} \dot{\phi}^{2} V}{\left(\dot{\phi}^{2}+2 a^{2} V\right) \frac{\mathrm{d} V}{\mathrm{~d} \phi}}
$$

$\mathrm{e}$

$$
\dot{\varphi}=\frac{\delta_{c} \dot{\phi}^{3}}{\dot{\phi}^{2}+2 a^{2} V} .
$$

Dessa maneira, o campo perturbado depende de quantidades que podem ser determinadas através de observações cosmológicas.

Esse formalismo estende o conjunto de parâmetros presentes no modelo padrão da cosmologia. Por exemplo, se o potencial do campo escalar for dado por uma forma funcional do tipo exponencial,

$$
V(\phi)=V_{0} e^{-\kappa \lambda \phi}
$$

onde $\kappa=\sqrt{8 \pi G}$, temos que os novos parâmetros são $V_{0}, \lambda$ e $Q$. No trabalho [61], por exemplo, o valor de $Q$ foi obtido ao comparar esse modelo com os dados da colaboração Planck para as anisotropias da radiação cósmica de fundo [62]. O valor encontrado, utilizando dados para BAO juntamente aos dados do Planck, foi $Q=0.036 \pm 0.016$ com $68 \%$ de confiança. Como essa constante de acoplamento está a mais de um sigma de distância do valor zero, vemos que, dentro do formalismo aqui exposto, uma interação no setor escuro é compatível com os dados observacionais disponíveis na literatura.

\subsection{Modelo de Yukawa para a Energia Escura Inte- ragindo com a Matéria Escura}

Para entendermos a interação entre os componentes do setor escuro de uma maneira mais fundamental, mas ainda assim fenomenológica, precisamos utilizar das ferramentas da teoria quântica de campos. Por essa razão, introduziremos nesta dissertação um modelo de interação no setor escuro construído através de primeiros princípios. Dessa maneira, para descrever a energia escura, consideraremos um campo escalar canônico e para descrever a matéria escura, consideraremos um campo fermiônico de spin- $\frac{1}{2}$. Esse campo fermiônico pode ser interpretado como um modelo efetivo para o neutralino, um dos principais candidatos para a matéria escura $[15,63]$. A interação entre a energia e a matéria escura será modelada pela interação de Yukawa, que é a única interação renormalizável entre um campo escalar e um campo 
fermiônico de spin- $\frac{1}{2}$ existente dentro do formalismo da teoria quântica de campos. Contudo, antes de introduzir nosso modelo, trataremos do formalismo necessário para introduzir férmions na presença de uma métrica curva.

\subsubsection{Formalismo das Tétradas}

Não existem representações do grupo das transformações gerais de coordenadas que se comportam como espinores sob a transformação do subgrupo de Lorentz. Assim, para podermos escrever uma ação escalar para um modelo que contém férmions, temos que adotar um formalismo no qual o campo de Dirac comporta-se como um escalar sob transformações gerais de coordenadas. Esse formalismo é conhecido como formalismo das tétradas [6,64].

De acordo com o Princípio de Equivalência, em cada ponto $x^{\mu}=X^{\mu}$ do espaçotempo, podemos considerar um sistema de coordenadas $y_{X}^{\alpha}$, que é localmente inercial em $x^{\mu}=X^{\mu}$. Nesse sistema localmente inercial, a métrica será a de Minkowski $\eta_{\alpha \beta}$. A métrica no sistema de coordenadas geral $x^{\mu}$ será dada por

$$
g_{\mu \nu}(x)=V_{\mu}^{\alpha}(x) V_{\nu}^{\beta}(x) \eta_{\alpha \beta},
$$

onde

$$
V_{\mu}^{\alpha}(X) \equiv\left(\frac{\partial y_{X}^{\alpha}}{\partial x^{\mu}}\right)_{x=X}
$$

Note que, para as coordenadas do referencial localmente inercial, estamos usando como índices as letras do início do alfabeto grego $(\alpha, \beta, \ldots)$, enquanto as letras do final do alfabeto grego são usadas para o sistema de coordenadas geral $(\mu, \nu, \ldots)$.

Considerando uma transformação no sistema geral de coordenadas, $x^{\mu} \rightarrow x^{\prime}{ }^{\prime}$ e mantendo fixas as coordenadas $y_{X}^{\alpha}$ do sistema localmente inercial, os objetos $V_{\mu}^{\alpha}(X)$ transformam-se como vetores covariantes, ou seja,

$$
V_{\mu}^{\prime \alpha}\left(X^{\prime}\right)=\frac{\partial x^{\nu}}{\partial x^{\prime \mu}} V_{\nu}^{\alpha}(X)
$$

Esse conjunto de quatro vetores é chamado de tétrada [6,63]. Sob uma transformação de Lorentz do sistema localmente inercial, $y_{X}^{\alpha} \rightarrow y_{X}^{\alpha}$, os campos da tétrada transformam-se como vetores de Lorentz contravariantes, ou seja,

$$
V_{\mu}^{\alpha}(X) \rightarrow \Lambda_{\beta}^{\alpha}(X) V_{\mu}^{\beta}(X)
$$

onde $\Lambda_{\beta}^{\alpha}(X)$ é a matriz da transformação de Lorentz.

Se contrairmos um vetor contravariante $A^{\mu} \operatorname{com} V_{\mu}^{\alpha}$, o objeto resultante, $A^{\alpha}=$ 
$V_{\mu}^{\alpha} A^{\mu}$, se transformará como um conjunto de quatro escalares sob uma transformação geral de coordenadas, enquanto que sob uma transformação de Lorentz do sistema localmente inercial, $A^{\alpha}$ se transformará como um vetor de Lorentz contravariante. Em geral, um tensor $n$ vezes contravariante e $m$ vezes covariante, $T_{\nu_{1} \ldots \nu_{m}}^{\mu_{1} \ldots \mu_{n}}$, ao ter seus $n$ índices contravariantes contraídos com $V_{\mu}^{\alpha}$ e os seus $m$ índices covariantes contraídos com a tétrada inversa $V_{\beta}^{\nu}$ se tornará um objeto que irá transformar-se como uma coleção de $n \times m$ escalares, sob uma transformação geral de coordenadas, e como um tensor de Lorentz $n$ vezes contravariante e $m$ vezes covariante, sob uma transformação de Lorentz no sistema localmente inercial. A tétrada inversa pode ser obtida abaixando o índice $\alpha$ e levantando o índice $\mu$ com as métricas correspondentes,

$$
V_{\beta}^{\mu}=\eta_{\alpha \beta} g^{\mu \nu} V_{\mu}^{\alpha}
$$

A ação dos campos representando a matéria depende dos próprios campos e também das suas derivadas. Consideremos um campo geral $\phi$, que se transforma, no espaço de Minkowski, como $\phi^{\prime}=D(\Lambda) \phi$, onde $D(\Lambda)$ é a representação do grupo de Lorentz correspondente. As derivadas de $\phi, \partial_{\alpha} \phi$, se transformarão como $\partial_{\beta}^{\prime} \phi^{\prime}=$ $\Lambda_{\beta}^{\alpha} D(\Lambda) \partial_{\alpha} \phi$. Assim, para que tenhamos uma ação escalar em um espaço-tempo curvo, tanto com relação à transformação do sistema geral de coordenadas, como com relação à transformação de Lorentz no sistema localmente inercial, precisamos definir uma derivada covariante $\nabla_{\alpha} \phi$, que sob transformações de Lorentz locais transformese como $\nabla_{\beta}^{\prime}=\Lambda_{\beta}^{\alpha} D(\Lambda) \nabla_{\alpha} \phi$. A derivada covariante que possui exatamente essa propriedade é $[6,64]$,

$$
\nabla_{\alpha} \equiv V_{\alpha}^{\mu}\left(\partial_{\mu}+\Gamma_{\mu}\right)=V_{\alpha}^{\mu}\left[\partial_{\mu}+\frac{1}{2} \sigma^{\gamma \beta} V_{\gamma}^{\nu}(x)\left(\tilde{\nabla}_{\mu} V_{\beta \nu}(x)\right)\right]
$$

Nessa expressão, temos que $\tilde{\nabla}_{\mu} V_{\beta \nu}(x) \equiv \partial_{\mu} V_{\beta \nu}(x)-\Gamma_{\mu \nu}^{\lambda} V_{\beta \lambda}(x)$ e $\sigma^{\alpha \beta}$ denota os geradores do grupo de Lorentz. Para os campos escalares, temos que $\sigma^{\alpha \beta}=0$ e $\nabla_{\alpha}=\partial_{\alpha}$. Para os espinores, $\sigma^{\alpha \beta}=\frac{1}{4}\left[\gamma^{\alpha}, \gamma^{\beta}\right]$, sendo $\gamma^{\alpha}$ as matrizes de Dirac.

Assim, para generalizarmos uma teoria quântica de campos para espaços-tempos curvos, temos que contrair todos os vetores e tensores com a tétrada (por exemplo, substituir $A^{\alpha}$ por $V_{\mu}^{\alpha} A^{\mu}$ ) e substituir as derivadas $\partial_{\alpha}$ por derivadas covariantes $\nabla_{\alpha}$. Desse modo, a ação obtida será um escalar, tanto para transformações gerais de coordenadas quanto para transformações de Lorentz locais. O tensor de energiamomento, por sua vez, pode ser definido como $[6,64]$

$$
T_{\mu \nu}(x) \equiv-\frac{V_{\alpha \mu}(x)}{\operatorname{det}[V(x)]} \frac{\delta S_{M}}{\delta V_{\alpha}^{\nu}}
$$


onde $S_{M}$ denota a ação dos campos de matéria e $\operatorname{det}[V(x)]=\sqrt{-g}$, com $g$ sendo o determinante de $g_{\mu \nu}(x)$. Usando (6.74), podemos obter o tensor de energia-momento para campos com qualquer spin em espaços curvos.

\section{Calculando as Tétradas}

Como nosso objetivo é utilizar o formalismo acima descrito para descrever campos de matéria interagindo no universo, consideraremos a métrica de fundo como sendo dada pela métrica de FRW plana

$$
\mathrm{d} s^{2}=-\mathrm{d} t^{2}+a^{2} \delta_{i j} \mathrm{~d} x^{i} \mathrm{~d} x^{j},
$$

de forma que $g_{00}=-1$ e $g_{i j}=a^{2} \delta_{i j}$. O sistema de equações (6.68) é indeterminado, visto que temos seis variáveis a mais do que o número de equações disponíveis. Dessa maneira, temos a liberdade de escolher algumas das tétradas. Escolhemos

$$
\begin{aligned}
& V_{0}^{i}=0 \text { para } i=1,2,3 \\
& V_{2}^{1}=V_{3}^{2}=V_{1}^{3}=0
\end{aligned}
$$

Com essa escolha o sistema é determinado e as tétradas restantes são dadas por

$$
\begin{aligned}
& V_{0}^{0}=1 \\
& V_{1}^{1}=V_{2}^{2}=V_{3}^{3}=a
\end{aligned}
$$

Todas as demais tétradas são iguais a zero.

\subsubsection{Modelo de Yukawa no Universo de FRW}

O modelo que desenvolveremos é composto por um campo escalar representando a energia escura e um campo fermiônico de spin- $\frac{1}{2}$ representando a matéria escura, sendo que a interação entre eles é a interação de Yukawa. Assim, a densidade lagrangiana do nosso modelo, utilizando o formalismo das tétradas, é dada por [65]

$$
\mathcal{L}_{M}=\sqrt{-g}\left[-\frac{1}{2} \partial_{\mu} \phi \partial^{\mu} \phi-V(\phi)+i\left(\bar{\Psi} \gamma^{\mu} \nabla_{\mu} \Psi-\bar{\Psi} \overleftarrow{\nabla}_{\mu} \gamma^{\mu} \Psi\right)-(M-\beta \phi) \bar{\Psi} \Psi\right]
$$


onde $\beta$ é a constante de acoplamento e $V(\phi)$ é o potencial do campo escalar canônico que, a princípio, pode ter qualquer forma funcional. As matrizes $\gamma^{\mu}$ são as matrizes de Dirac no espaço curvo e relacionam-se com as matrizes de Dirac comuns $\gamma^{\alpha}$ através da tétrada, $\gamma^{\mu}=V_{\alpha}^{\mu} \gamma^{\alpha}$. As matrizes $\gamma^{\mu}$ satisfazem a relação de anticomutação

$$
\left\{\gamma^{\mu}, \gamma^{\nu}\right\}=-2 g^{\mu \nu}(x)
$$

A ação da matéria será

$$
S_{M}=\int \mathrm{d}^{4} x \mathcal{L}_{M}(x)
$$

com a densidade de lagrangiana $\mathcal{L}_{M}(x)$ dada por (6.78). Efetuando a variação de $S_{M}$ com relação ao campo $\bar{\Psi}$, obtemos a equação de movimento para o campo de Dirac $\Psi$

$$
i \gamma^{\mu} \nabla_{\mu} \Psi-M_{\mathrm{ef}} \Psi=0
$$

onde $M_{\mathrm{ef}} \equiv M-\beta \phi$ é a massa efetiva do férmion. Do mesmo modo, variando $S_{M}$ com respeito a $\Psi$, obtemos a equação para o campo de Dirac adjunto $\bar{\Psi}$

$$
i\left(\nabla_{\mu} \bar{\Psi}\right) \gamma^{\mu}+M_{\mathrm{ef}} \bar{\Psi}=0
$$

As equações acima são as equações de Dirac e sua adjunta generalizadas para um espaço-tempo curvo e com uma interação de Yukawa com o campo escalar $\phi[64,65]$. Utilizando essas equações, podemos mostrar que a corrente $j^{\mu}(x)=\sqrt{-g} \bar{\Psi} \gamma^{\mu} \Psi$ conserva-se, ou seja, temos que

$$
\partial_{\mu} j^{\mu}(x)=0
$$

Variando $S_{M}$ com respeito a $\phi$, obtemos a equação de movimento para o campo escalar

$$
\nabla_{\mu} \partial^{\mu} \phi-V^{\prime}(\phi)+\beta \bar{\Psi} \Psi=0
$$

onde $V^{\prime}(\phi)=\mathrm{d} V(\phi) / \mathrm{d} \phi$.

Usando a equação (6.74), podemos calcular o tensor de energia-momento para nosso modelo. Obtemos, então, que $[63,64]$

$$
T_{\mu \nu}=T_{\mu \nu}^{\phi}+T_{\mu \nu}^{\Psi}
$$

onde 


$$
T_{\mu \nu}^{\phi}=\partial_{\mu} \phi \partial_{\nu} \phi-g_{\mu \nu}\left[g^{\alpha \beta} \frac{\partial_{\alpha} \phi \partial_{\beta} \phi}{2}+V(\phi)\right]
$$

$\mathrm{e}$

$$
T_{\mu \nu}^{\Psi}=\frac{i}{4}\left[\left(\nabla_{\mu} \bar{\Psi}\right) \gamma_{\nu} \Psi+\left(\nabla_{\nu} \bar{\Psi}\right) \gamma_{\mu} \Psi-\bar{\Psi} \gamma_{\mu} \nabla_{\nu} \Psi-\bar{\Psi} \gamma_{\nu} \nabla_{\mu} \Psi\right]
$$

Até o momento, o tratamento é totalmente geral e pode ser aplicado para qualquer sistema físico. Agora, contudo, consideraremos um universo homogêneo e isotrópico, caso em que o tensor de energia-momento representando a matéria total do universo em larga escala é dado pelo tensor de energia-momento de um fluido ideal,

$$
T_{\mu \nu}=(P+\rho) u_{\mu} u_{\nu}+g_{\mu \nu} P
$$

Em um sistema de referência que se move com a velocidade do fluido, temos que $u^{\mu}=\left(1 / \sqrt{-g_{00}}, 0,0,0\right)$. Assim, podemos identificar diretamente que

$$
\rho=-T_{0}^{0} \quad \text { e } \quad P=\frac{1}{3} T_{i}^{i} .
$$

Assim, considerando que nossos campos podem ser descritos por um fluido ideal, obtemos de $T_{\mu \nu}^{\phi}$ que

$$
\rho^{\phi}=-\frac{1}{2}\left(g^{0 \nu} \partial_{0} \phi \partial_{\nu} \phi-g^{i \nu} \partial_{i} \phi \partial_{\nu} \phi\right)+V(\phi)
$$

$\mathrm{e}$

$$
P^{\phi}=-\frac{1}{2}\left(g^{0 \nu} \partial_{0} \phi \partial_{\nu} \phi-g^{i \nu} \partial_{i} \phi \partial_{\nu} \phi\right)-V(\phi) .
$$

Já de $T_{\mu \nu}^{\Psi}$, obtemos que

$$
\rho^{\Psi}=M_{\mathrm{ef}} \bar{\Psi} \Psi+\frac{i}{2}\left[\left(\nabla^{i} \bar{\Psi}\right) \gamma_{i} \Psi-\bar{\Psi} \gamma^{i} \nabla_{i} \Psi\right]
$$

$\mathrm{e}$

$$
P^{\Psi}=\frac{i}{6}\left[\left(\nabla_{i} \bar{\Psi}\right) \gamma^{i} \Psi-\bar{\Psi} \gamma^{i} \nabla_{i} \Psi\right] .
$$

Para $M_{\mathrm{ef}}=0$, vemos que obtemos uma equação de estado de um gás ideal relativístico para o campo fermiônico, $P^{\Psi}=\rho^{\Psi} / 3$.

Para um universo de FRW, os campos são homogêneos em larga escala, dessa forma temos que $\partial_{i} \phi=0, \partial_{i} \Psi=\partial_{i} \bar{\Psi}=0$. Como para a métrica de FRW temos que $\nabla_{i} \Psi=\partial_{i} \Psi-\dot{a} \sigma^{0 i} \Psi$ e que $\nabla_{i} \bar{\Psi}=\partial_{i} \bar{\Psi}-\dot{a} \sigma^{0 i} \bar{\Psi}$, podemos escrever a densidade de energia e a pressão dos campos como 


$$
\begin{gathered}
\rho^{\phi}=\frac{\dot{\phi}^{2}}{2}+V(\phi), \\
P^{\phi}=\frac{\dot{\phi}^{2}}{2}-V(\phi), \\
\rho^{\Psi}=M_{\mathrm{ef}} \bar{\Psi} \Psi, \\
P^{\Psi}=0 .
\end{gathered}
$$

Note que o férmion comporta-se como um fluido de pressão zero, isto é, comporta-se como a matéria escura fria. Também temos que

$$
\omega^{\phi} \equiv \frac{P^{\phi}}{\rho^{\phi}}=\frac{\dot{\phi}^{2}-2 V}{\dot{\phi}^{2}+2 V} .
$$

A equação de movimento para o campo escalar, (6.84), reduz-se, em um universo de FRW, a

$$
\ddot{\phi}+3 H \dot{\phi}+\frac{\mathrm{d} V}{\mathrm{~d} \phi}-\beta \bar{\Psi} \Psi=0,
$$

enquanto a lei de conservação (6.83) reduz-se a

$$
\frac{\mathrm{d}\left(a^{3} \Psi^{\dagger} \Psi\right)}{\mathrm{d} t}=0
$$

Derivando as equações (6.94) e (6.96) em relação ao tempo e usando as equações de movimento dos respectivos campos, obtemos as equações de balanço para os fluidos interagentes

$$
\dot{\rho}^{\phi}+3 H \rho^{\phi}\left(\omega^{\phi}+1\right)=\beta \dot{\phi} \bar{\Psi} \Psi=Q_{0}
$$

e

$$
\dot{\rho}^{\Psi}+3 H \rho^{\Psi}=-\beta \dot{\phi} \bar{\Psi} \Psi=-Q_{0} .
$$

O termo de interação dessas equações, $Q_{0}=\beta \dot{\phi} \bar{\Psi} \Psi$, pode ser reescrito como $[58,66]$

$$
Q_{0}=\beta \dot{\phi} \bar{\Psi} \Psi=-\frac{\partial \ln M_{\mathrm{ef}}}{\partial \phi} \rho^{\Psi} \dot{\phi}=\frac{\beta}{M-\beta \phi} \rho^{\Psi} \dot{\phi}
$$

onde $M_{\mathrm{ef}}=M-\beta \phi$ e utilizamos a definição (6.96) para a densidade do campo fermiônico. Com essa definição para o acoplamento entre os componentes do setor 
escuro, podemos considerar a matéria escura como sendo totalmente descrita por um fluido perfeito, visto que somente a densidade $\rho^{\Psi}$ aparece nas equações homogêneas. A equação de movimento para o campo escalar (6.102) pode, portanto, ser reescrita como

$$
\ddot{\phi}+3 H \dot{\phi}+\frac{\mathrm{d} V}{\mathrm{~d} \phi}+\frac{\partial \ln M_{\mathrm{ef}}}{\partial \phi} \rho^{\Psi}=0
$$

ou seja,

$$
\ddot{\phi}+3 H \dot{\phi}+\frac{\mathrm{d} V}{\mathrm{~d} \phi}-\frac{\beta}{M-\beta \phi} \rho^{\Psi}=0 .
$$

Assim, nosso modelo funcionará como um híbrido entre o formalismo de campos com o formalismo de fluidos. A reinterpretação do campo fermiônico como um fluido perfeito facilitará nossos estudos do universo no contexto da teoria de perturbações lineares. Dessa maneira, consideraremos o universo como sendo composto por um campo escalar canônico representando a energia escura e por um fluido de pressão e velocidade do som zero representando a matéria escura. A interação entre eles será dada por um termo do tipo

$$
Q^{\mu}=-\frac{\partial \ln M_{\mathrm{ef}}}{\partial \phi} \rho_{c} \partial^{\mu} \phi=\frac{\beta}{M-\beta \phi} \rho_{c} \partial^{\mu} \phi
$$

onde renomeamos $\rho^{\Psi}$ como $\rho_{c}$.

As equações de balanço, portanto, podem ser escritas como

$$
\nabla_{\nu} T_{\phi}^{\mu \nu}=Q^{\mu}=\frac{\beta}{M-\beta \phi} \rho_{c} \nabla^{\mu} \phi
$$

e

$$
\nabla_{\nu} T_{c}^{\mu \nu}=-Q^{\mu}=-\frac{\beta}{M-\beta \phi} \rho_{c} \nabla^{\mu} \phi
$$

onde $T_{c}^{\mu \nu}$ é o tensor de energia-momento de um fluido ideal, $T_{c}^{\mu \nu}=\left(\rho_{c}+P_{c}\right) u^{\mu} u^{\nu}+$ $P_{c} g^{\mu \nu}$.

Para estudar essas equações em nível perturbativo, consideraremos novamente a métrica de FRW perturbada no gauge síncrono que é dada pela equação (6.53). Novamente, no que segue, toda derivada "temporal" será em relação ao tempo conforme.

Utilizando essa métrica podemos perturbar linearmente as equações (6.107) e (6.108). O campo escalar será considerado como composto por uma parte homogênea e uma parte perturbada: $\phi(t)+\varphi(\mathbf{x}, t)$. Obtemos, dessa maneira, as equações, no espaço de Fourier, para o contraste de densidade, $\delta_{c}=\delta \rho_{c} / \rho_{c}$, e a divergência da 
velocidade, $\theta_{c}=\nabla_{i} v_{c}^{i}$, da matéria escura com equação de estado $\omega=0$ e velocidade do $\operatorname{som} c_{s}^{2}=0$,

$$
\begin{gathered}
\dot{\delta}_{c}=-\theta_{c}-\frac{\dot{h}}{2}-\frac{\beta}{M-\beta \phi} \dot{\varphi}+\frac{\beta^{2}}{(M-\beta \phi)^{2}} \dot{\phi} \varphi \\
\dot{\theta}_{c}=-\mathcal{H} \theta_{c}+\frac{\beta}{M-\beta \phi} \theta_{c} \dot{\phi}-k^{2} \frac{\beta}{M-\beta \phi} \varphi,
\end{gathered}
$$

onde $\mathcal{H}=\frac{1}{a} \frac{\mathrm{d} a}{\mathrm{~d} \tau}$.

A equação de evolução para a perturbação do campo escalar, $\varphi$, por sua vez, pode ser obtida, considerando o termo temporal da equação (6.107) perturbada linearmente,

$$
\ddot{\varphi}+2 \mathcal{H} \dot{\varphi}+k^{2} \varphi+a^{2} \frac{\mathrm{d}^{2} V}{\mathrm{~d} \phi^{2}} \varphi+\frac{\dot{h} \dot{\phi}}{2}=-a^{2} \frac{\beta^{2}}{(M-\beta \phi)^{2}} \varphi \rho_{c}+a^{2} \frac{\beta}{M-\beta \phi} \rho_{c} \delta_{c} .
$$

As condições iniciais para o campo perturbado podem ser obtidas, como no caso da quintessência acoplada, da suposição que as perturbações sejam adiabáticas e que a entropia intrínseca do campo escalar seja zero, isto é, exigimos que as relações (6.65) e (6.66) sejam válidas.

\subsubsection{Comparação com Dados Observacionais}

Com a teoria do modelo de Yukawa desenvolvida, nosso próximo objetivo é limitar os parâmetros do modelo de Yukawa através de observações cosmológicas para verificar sua viabilidade. Para isso, precisamos de uma versão modificada do código CAMB [43] que inclua um campo escalar e uma interação entre esse campo e a matéria escura. Também precisamos definir uma forma funcional para o potencial do campo escalar. Um exemplo seria um potencial exponencial do tipo dado pela equação (6.67). Esse potencial é largamente utilizado em estudos de quintessência [55]. Com essa escolha, o conjunto de novos parâmetros que estenderia o modelo $\Lambda$ CDM seria o acoplamento de Yukawa $\beta$, a massa da matéria escura $M$, a amplitude do potencial $V_{0}$ e a constante adimensional $\lambda$. Para o acoplamento de Yukawa esperamos que $\beta^{2} / 4 \pi \simeq 3 e^{2} / 4 \pi=3 \alpha$, onde $\alpha$ é a constante de estrutura fina da eletrodinâmica quântica [67]. Para a massa da matéria escura, esperamos que $M$ fique na faixa prevista para a massa do neutralino que está entre 10 e $10000 \mathrm{GeV}$ [15]. Contudo, como podemos ver das equações (6.109), (6.110) e (6.111), os parâmetros $\beta$ e $M$ são degenerados. Em todas as equações de balanço somente a razão $\beta / M$ ou $M / \beta$ aparece. Desse modo, podemos eliminar um desses parâmetros e escrever 
nossas equações em função da razão da constante de acoplamento pela massa do férmion, por exemplo. Com isso, nosso modelo fica mais simples do ponto de vista bayesiano, com o revés de não podermos mais determinar inequivocamente a massa do férmion e a constante de acoplamento. De qualquer modo, com esses priors para nossos parâmetros, podemos fazer uma análise bayesiana para determinar os limites observacionais para nossos parâmetros ao comparar o modelo de Yukawa com, por exemplo, os dados da colaboração Planck [62].

Essa parte do trabalho está sendo desenvolvida. Como a interação do setor escuro do modelo de Yukawa é divergente quando $\beta \phi=M$ (ver equação (6.103)), problemas numéricos surgem quando utilizamos o código CAMB para resolver as equações de evolução para o universo. Todavia, tais problemas podem ser contornados, de forma que trabalhos futuros poderão ser realizados utilizando o modelo de Yukawa que foi introduzido nesta dissertação. 



\section{Capítulo 7}

\section{Conclusão}

O objetivo desta dissertação foi estudar, em um universo de Friedmann-RobertsonWalker (FRW) linearmente perturbado, modelos em que a matéria escura e a energia escura interagem. Para isso, primeiramente, desenvolvemos os fundamentos da teoria de perturbação linear aplicada a um sistema relativístico, que no nosso caso correspondeu ao universo inteiro. Então, os modelos para o setor escuro interagente foram discutidos. Dois deles, o de fluidos interagentes e o oriundo de modelos de $f(R)$, já foram exaustivamente discutidos na literatura. Nosso objetivo foi utilizá-los como guias, laboratórios teóricos, para desenvolvermos as ferramentas necessárias para estudarmos o modelo que introduzimos nessa dissertação e que alcunhamos de modelo de Yukawa.

Esse modelo parte do pressuposto que uma possível interação entre os componentes do setor escuro do universo pode ser derivada de primeiros princípios e de uma teoria física conhecida. Para isso, utilizamos a lagrangiana renormalizável da teoria quântica de campos que envolve campos escalares e campos fermiônicos de spin- $\frac{1}{2}$. Como a energia escura, quando não interpretada como uma constante cosmológica, é, na maioria das vezes, descrita na literatura como um campo escalar canônico, adotamos em nosso modelo essa interpretação. A matéria escura pode ser representada, por sua vez, por um campo fermiônico de spin- $\frac{1}{2}$. Essa escolha pode ser vista como uma descrição efetiva para as partículas fracamente interagentes que são utilizadas para descrever a matéria escura fria. Como na teoria quântica de campos o único termo de interação renormalizável entre um campo escalar e um campo fermiônico de spin- $\frac{1}{2}$ é o termo de Yukawa, propomos essa interação para o setor escuro.

As equações de movimento e o tensor de energia-momento foram, então, obtidos com o uso do formalismo das tétradas. Estudando o comportamento dos campos em um universo de FRW plano, pudemos reescrever nosso sistema de equações utilizando 
a linguagem de fluidos perfeitos. As equações de balanço para os fluidos puderam, então, ser escritas em um universo de FRW linearmente perturbado.

Como foi dito no corpo desta dissertação, a comparação de nosso modelo com observações, o próximo passo lógico no desenvolvimento de nosso trabalho, não pôde ser realizada em tempo devido à problemas computacionais e numéricos que encontramos no desenvolvimento de nosso código para o CAMB modificado que será utilizado para comparar nosso modelo com os dados do Planck para as anisotropias da radiação cósmica de fundo. Contudo, tal trabalho já está em andamento e, provavelmente, logo teremos resultados no que concerne os valores para os parâmetros que introduzimos ao estendermos o modelo padrão da cosmologia quando consideramos uma interação no setor escuro.

Outros trabalhos poderão ser desenvolvidos utilizando o modelo de Yukawa descrito nesta dissertação. Pretendemos, por exemplo, compará-lo com dados de lookback time, redshift-space distortions e linhas de 21-cm do hidrogênio. Também, pretendemos desenvolver uma teoria que expande as ideias presentes no modelo de Yukawa ao fazer com que a matéria escura seja descrita, não por um campo fermiônico efetivo, mas por um campo supersimétrico que, acredita-se, seja a origem física para a matéria escura. 


\section{Bibliografia}

[1] He, J. H. e B. Wang. JCAP, 10, 2008.

[2] Valiviita, J., E. Majerotto e R. Maartens. JCAP, 208, 2008.

[3] Olivares, G., F. Atrio-Barandela e D. Pavon. Phys. Rev. D, 77, 2008.

[4] He, J. H., B. Wang e E. Abdalla. Phys. Rev. D., 83, 2011.

[5] Abdalla, E., L. R. Abramo, L. Sodre e B. Wang. Phys. Lett. B, 673, 2009.

[6] Weinberg, S.: Gravitation and Cosmology. Wiley and Sons, 1972.

[7] Bennett, C. L. et al. ApJS, 208, 2013.

[8] Dodelson, S.: Modern Cosmology. Academic Press, 2003.

[9] Freedman, W. L. et al. Astrophys. J., 47, 2001.

[10] Weinberg, S.: Cosmology. 2008.

[11] Mangano, G., G. Miele, S. Pastor e M. Peloso. Phys. Lett. B, 534, 2002.

[12] Burles, S., K. M. Nollett e M. S. Turner. Astrophys. J., 552, 2001.

[13] Hu, W., N. Sugiyama e J. Silk. Nature, 386, 1997.

[14] Schneider, P.: Extragalactic Astronomy and Cosmology - An Introduction. Springer, 2006.

[15] Bertone, G. et al. Phys. Rept., 405, 2005.

[16] Copeland, E. J., M. Sami e S. Tsujikawa. International Journal of Modern Physics D, 15, 2006.

[17] Malik, K. A. e D. R. Matravers. Class. Quant. Grav., 25, 2008.

[18] Amendola, L. e S. Tsujikawa: Dark Energy: Theory and Observations. Cambridge University Press, 2010. 
[19] Arfken G. B. e Weber H. J.: Mathematical Methods for Physicists. Elsevier Academic Press, 2005.

[20] Linder, E. V. Phys. Rev. D, 72, 2005.

[21] Mandl, F. e G. Shaw: Quantum Field Theory. John Wiley and Sons, 2010.

[22] Mukhanov, V.: Physical Foundations of Cosmology. Cambridge University Press, 2005.

[23] Kodama, H. e M. Sasaki. Prog. Theor. Phys. Suppl., 78, 1988.

[24] Perlmutter, S. J. et al. Nature, 391, 1998.

[25] Riess, A. G. et al. Astron. J., 116, 1998.

[26] Carretta, E., R. G. Gratton, G. Clementini e F. Pecci. Astrophys. J., 553, 2000.

[27] Jimenez, R., P. Thejll, U. Jorgensen, J. MacDonald e B. Pagel. Mon. Not. Roy. Astron. Soc., 282, 1996.

[28] Hamuy, M. et al. Astron. J., 112, 1996.

[29] Elgarøy, Ø. e T. Multamäki. A\&A, 471, 2007.

[30] Weinberg, S. Rev. Mod. Phys., 61, 1989.

[31] Clifton, T., P. G. Ferreira, A. Padilla e C. Skordis. Physics Reports, 513, 2012.

[32] Barrow, J. e F. Tipler: The Cosmological Anthropic Principle. Oxford University Press, 1988.

[33] Caldwell, R. R., R. Dave e P. J. Steinhardt. Phys. Rev. Lett., 80, 1998.

[34] Baumann, D. arXiv: 0907.5424.

[35] Copeland, E. J., A. R. Liddle e D. Wands. Phys. Rev. D, 57, 1998.

[36] Percival, I. e D. Richards: Introduction to Dynamics. Cambridge University Press, 1999.

[37] Huey, G. e B. D. Wandelt. Phys. Rev. D., 74, 2006.

[38] Tsujikawa, S. e M. Sami. Phys. Lett. B, 603, 2003.

[39] Wang, B., Chi Yong Lin, D. Pavon e E. Abdalla. Phys. Lett. B, 662, 2008. 
[40] Wang, B. e D. Pavon. Gen. Rel. Grav., 41, 2009.

[41] Atkins, P. W.: The Elements of Physical Chemistry. Oxford University Press, 1993.

[42] Kodama, H. e M. Sasaki. Prog. Theor. Phys. Suppl., 78, 1984.

[43] http://camb.info/.

[44] He, J. H., B. Wang e P. Zhang. Phys. Rev. D, 80, 2009.

[45] Bardeen, J. M. Phys. Rev. D, 22, 1980.

[46] Malik, K. A., D. Wands e C. Ungarelli. Phys. Rev. D, 67, 2003.

[47] Gordon, C. e W. Hu. Phys. Rev. D, 70, 2004.

[48] He, J. H., B. Wang e E. Abdalla. Phys. Lett. B, 671, 2009.

[49] De Felice, A. e S. Tsujikawa. Living Rev. Relativity, 13, 2010.

[50] Starobinsky, A. A. J. Exp. Theor. Phys. Lett., 86, 2007.

[51] Brans, C. e R. H. Dicke. Phys. Rev., 124, 1961.

[52] Wald, R. M.: General Relativity. University of Chicago Press, 1984.

[53] Faraoni, V. Phys. Rev. D, 80, 2009.

[54] Amendola, L., D. Polarski e S. Tsujikawa. Phys. Rev. Lett., 98, 2007.

[55] Amendola, L. Phys. Rev. D, 62, 1999.

[56] Gasperini, M. e G. Veneziano. Phys. Rept., 373, 2003.

[57] Khoury, J. e A. Weltman. Phys. Rev. Lett., 93, 2004.

[58] Xia, Jun Qing. Phys. Rev. D, 80, 2009.

[59] Chongchitnan, S. Phys. Rev. D, 79, 2009.

[60] D. Wands, K. A. Malik, D. H. Lyth e A. R. Liddle. Phys. Rev. D, 62, 2000.

[61] Pettorino, V. Phys. Rev. D, 88, 2013.

[62] Ade, P. A. R. et al. arXiv: 1303.5076.

[63] Micheletti, S., E. Abdalla e B. Wang. Phys. Rev. D, 79, 2009. 
[64] Birrell, N. D. e P. C. W. Davies: Quantum Fields in Curved Space. Cambridge University Press, 1986.

[65] Srednicki, M.: Quantum Field Theory. Cambridge University Press, 2007.

[66] Amendola, L., V. Pettorino, C. Quercellini e A. Vollmer. Phys. Rev. D, 85, 2012.

[67] Abdalla, E. e B. Wang. Phys. Lett B, 51, 2007. 